\title{
34. MIDDLE TO LATE QUATERNARY SEDIMENT FLUXES IN THE LABRADOR SEA, ODP LEG 105, SITE 646:A SYNTHESIS OF ROCK-MAGNETIC, OXYGEN-ISOTOPIC, CARBONATE, AND PLANKTONIC FORAMINIFERAL DATA ${ }^{1}$
}

\author{
Frank R. Hall, ${ }^{2}$ Jan Bloemendal, ${ }^{2}$ John W. King, ${ }^{2}$ Michael A. Arthur, ${ }^{2}$ and Ali E. Aksu ${ }^{3}$
}

\begin{abstract}
We examine rock-magnetic, carbonate, and planktonic foraminiferal fluxes to identify climatically controlled changes of terrigenous and pelagic sedimentation at Ocean Drilling Program (ODP) Site 646 (the Labrador Sea). Terrigenous sediments are brought to the site principally by bottom currents. We use a rock-magnetic parameter sensitive to changes in magnetic mineral grain size, the ratio of anhysteretic susceptibility to low-field magnetic susceptibility $\left(X_{A R M} / X\right)$, to monitor changes in bottom-current intensity over time, with large values of $X_{A R M} / X$ (finer-grained magnetic minerals) indicating weaker bottom currents. A second rock-magnetic parameter, magnetic mineral accumulation rate $\left(K_{\mathrm{ar}}\right)$ was used to indicate variations in terrigenous flux. Planktonic foraminiferal and carbonate accumulation rates $\left(P f_{a r}\right.$ and $\left.\mathrm{CaCO}_{3 a r}\right)$ are used as indicators of pelagic flux.

Absolute age assignments are based on correlation between the planktonic foraminiferal oxygen-isotope variations for Site 646 and the SPECMAP master oxygen-isotope curve. Cross-correlation analyses of the parameters that we studied with respect to the SPECMAP curve suggest that from oxygen-isotope stages 21 to 11 , sedimentation rate, $K_{\text {ar }}$, $\mathrm{X}, \mathrm{CaCO}_{3 a r}$, and $P f_{a r}$ were at their maximums, whereas $X_{A R M} / X$ was at its minimum during peak interglacials (i.e., 0 k.y. lag time with respect to minimum ice volume). However, all parameters we examined lag behind minimum ice volume from stages 11 to 1 , indicating a change in timing of both pelagic and terrigenous fluxes at approximately 400 k.y. BP. The negative correlation coefficient between $X_{A R M} / X$ and the SPECMAP curve further suggest that finer-grained magnetic minerals are deposited during glacial periods, which probably reflects weaker bottom currents.

The shift observed in the lag times of parameters examined with respect to the SPECMAP record is attributed to a change in significance of orbital parameters. Spectral results exhibit strong power in eccentricity (about 100 k.y.) throughout the record. $K_{\mathrm{ar}} X, \mathrm{CaCO}_{3 a r}$, and $P f_{a r}$ show significant power in obliquity (about 41 k.y.), whereas $X_{A R M} / X$ shows significant power at 73 k.y. from stages 21 to 11 . The 73-k.y. period in $X_{A R M} / X$ is near the difference tone of obliquity and eccentricity: $1 / 43-1 / 102=1 / 69 . K_{a r}$ and $X_{A R M} / X$ show power only in eccentricity from stages 11 to 1 . $X$ and $P f_{a r}$ show significant power in precession (about 18 and 22 k.y.) whereas $\mathrm{CaCO}_{3 a r}$ has power at 34 k.y., which could be a combination of precession and obliquity. The shift in power of orbital parameters may by attributed to the effect of the about 413-k.y. signal of eccentricity.
\end{abstract}

\section{INTRODUCTION}

We examine climatic controls on sediment flux in the northern Labrador Sea, an area of high climatic sensitivity during the middle to late Quaternary. For this study, we used several types of data: rock-magnetic properties, the oxygen isotopic composition of planktonic foraminifers, and the concentrations and accumulation rates of carbonate and planktonic foraminifers.

Site 646 is located in the North Atlantic, just north of the Eirik Drift $\left(58^{\circ} 13^{\prime} \mathrm{N}, 48^{\circ} 22^{\prime}\right.$ W: Fig. 1). The site was probably covered by ice during the last glacial interval (Ruddiman and McIntyre, 1979): clearly, the advance and retreat of northern high-latitude ice is important for controlling deposition in this region.

Fine-grained sediments of the Eirik Drift are transported to the site by bottom-flowing contour currents. These sediments are highly bioturbated, which commonly destroys the sediment fabric and structure usually associated with current deposition (Chough and Hesse, 1985).

With the exception of in-situ deposition, such as bacterial magnetite (Kirschvink and Chang, 1984; Petersen et al., 1986),

\footnotetext{
${ }^{1}$ Srivastava, S. P., Arthur, M., Clement, B., et al., 1989. Proc. ODP, Sci. Results, 105: College Station, TX (Ocean Drilling Program).

2 Graduate School of Oceanography, University of Rhode Island, Narragansett, RI 02882.

${ }^{3}$ Earth Sciences Department, Memorial University of Newfoundland, St. John's, Newfoundland A1B 3X5, Canada.
}

magnetic minerals are deposited on the seafloor by the same processes that control the deposition of other terrigenous minerals. Thus, one can use the flux and particle size of magnetic minerals at Site 646 as proxy indicators of changing terrigenous sedimentary flux.

The use of rock-magnetic techniques to elucidate sedimentary processes is relatively new to oceanographic studies. Robinson (1986) and Bloemendal et al. (1988) demonstrated that downcore variations in rock-magnetic parameters correlate with oxygen-isotope stratigraphy of upper Quaternary sediments from the mid-North Atlantic and equatorial eastern Atlantic, respectively. We have applied the techniques of Bloemendal et al. (1988) to samples cored with the advanced piston corer (APC) from ODP Site 646.

\section{METHODS}

Time Control

To compare the various types of data used here directly with the planktonic foraminferal oxygen-isotope curve of Site 646 (Aksu et al., this volume), we used only results from those samples within the same core sections sampled by Aksu et al. (this volume) (Table 1).

To develop an age model for Site 646, we identified 45 events (Imbrie et al., 1986; Prell et al., 1987) within the oxygen-isotope record (Aksu et al., this volume). This oxygen-isotope record is based on the planktonic foraminifer Neogloboquadrina pachyderma (sinistral). We assigned ages based on correlation of the 45 events with the SPECMAP oxygen-isotope master curve of Imbrie et al. (1984) (Table 2). We then assigned ages to individual samples by linearly interpolating sample depths between these 45 tie-points; values of the parameters used in this study then were linearly interpolated to equal intervals of time (2000 yr). 


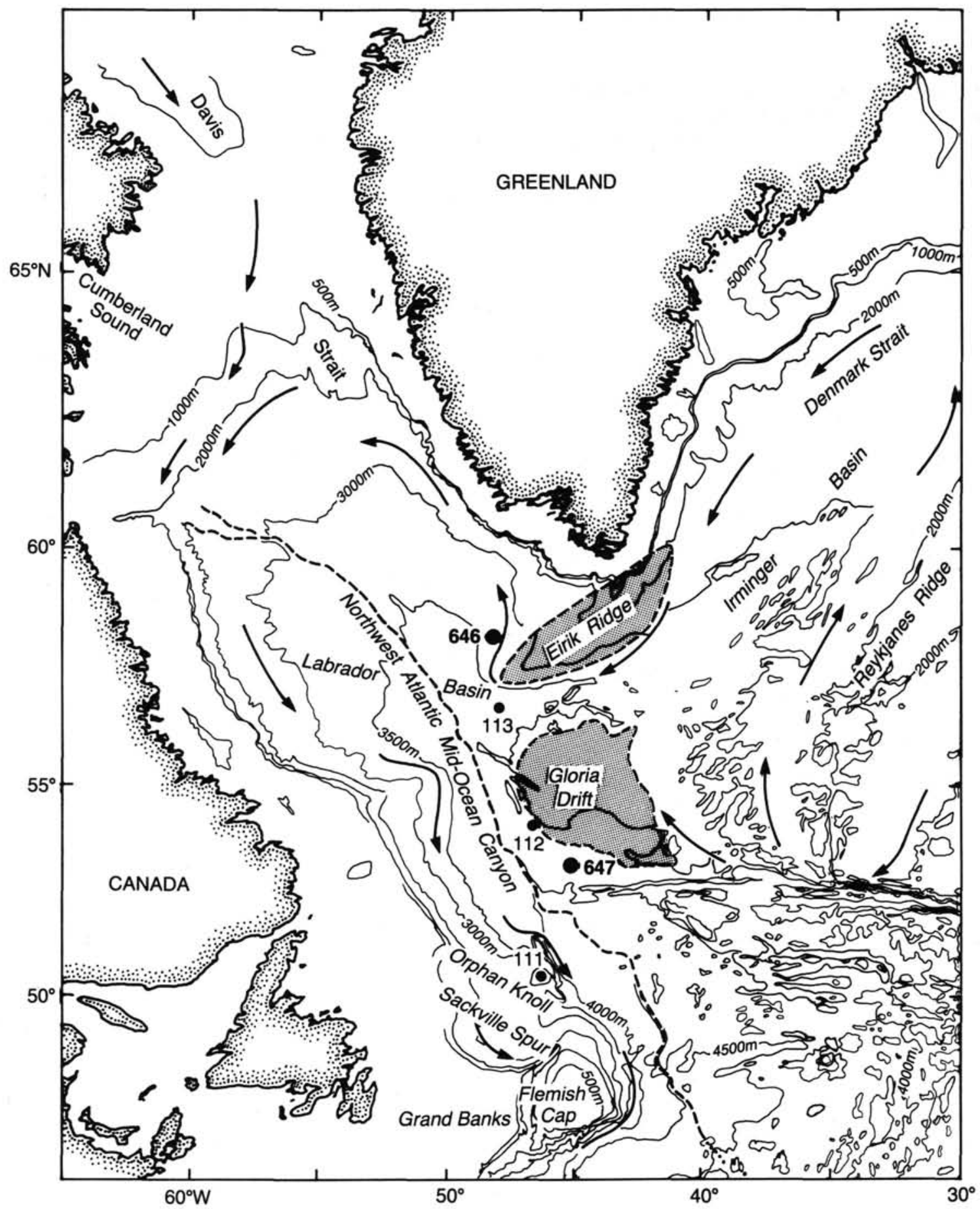

Figure 1. Location map of Site 646.

Sedimentation Rate $(S)$

Sedimentation rates were calculated by the equation:

$$
S=\frac{\left(D_{1}-D_{2}\right)}{\left(A_{1}-A_{2}\right)}
$$

where $D_{1}$ and $D_{2}$ are consecutive depths, and $A_{1}$ and $A_{2}$ are consecutive ages for the 45 events identified from the Site 646 oxygen-isotope record. The sedimentation rate was then converted to bulk-sediment accumulation rate $(B A R)$ by the equation:

$$
B A R=S\{B-[(P / 100) \times 1.01]\},
$$

where 1.01 is the density of seawater, $B$ is the wet bulk density, and $P$ is the percent of porosity. Bulk density and porosity were determined for each age interval by interpolating between data points given in the "Site 646" chapter (Srivastava, Arthur et al., 1987). Because of the low sampling density of physical property data, all values were honored.

\section{Rock-Magnetic Parameters}

The methods for determining rock-magnetic parameters are described in Hall and King (this volume), and values for rock magnetic data are 
Table 1. Intervals cores used in this study and top and bottom ages, based on interpolation between events given in Table 2.

\begin{tabular}{|c|c|c|}
\hline $\begin{array}{l}\text { Core/section } \\
\text { interval }(\mathrm{cm})\end{array}$ & $\begin{array}{l}\text { Depth } \\
\text { (mbsf) }\end{array}$ & $\begin{array}{l}\text { Age } \\
\text { (k.y.) }\end{array}$ \\
\hline $105-646 \mathrm{~A}-1 \mathrm{H}-1,0$ & 0 & 0 \\
\hline $646 \mathrm{~A}-1 \mathrm{H}-4,50$ & 5.00 & 49.66 \\
\hline $646 \mathrm{~B}-1 \mathrm{H}-4,100$ & 5.00 & 49.66 \\
\hline $646 \mathrm{~B}-1 \mathrm{H}-6,150$ & 8.50 & 87.29 \\
\hline $646 \mathrm{~B}-2 \mathrm{H}-3,50$ & 8.00 & 75.80 \\
\hline 646B-2H-6, 70 & 12.70 & 141.86 \\
\hline $646 \mathrm{~A}-2 \mathrm{H}-2,100$ & 12.70 & 141.86 \\
\hline $646 \mathrm{~A}-2 \mathrm{H}-7,45$ & 19.65 & 228.94 \\
\hline $646 \mathrm{~B}-3 \mathrm{H}-4,120$ & 20.30 & 235.78 \\
\hline $646 \mathrm{~B}-3 \mathrm{H}-7,50$ & 24.10 & 263.94 \\
\hline $646 \mathrm{~A}-3 \mathrm{H}-4,0$ & 24.50 & 267.26 \\
\hline $646 \mathrm{~A}-3 \mathrm{H}-6,30$ & 27.80 & 318.82 \\
\hline $646 \mathrm{~B}-4 \mathrm{H}-1,120$ & 27.20 & 310.92 \\
\hline $646 \mathrm{~B}-4 \mathrm{H}-5,50$ & 32.50 & 382.39 \\
\hline $646 \mathrm{~A}-4 \mathrm{H}-2,120$ & 32.50 & 382.39 \\
\hline $646 \mathrm{~A}-4 \mathrm{H}-6,150$ & 38.80 & 455.38 \\
\hline $646 \mathrm{~A}-5 \mathrm{H}-1,0$ & 39.30 & 463.47 \\
\hline $646 \mathrm{~A}-5 \mathrm{H}-6,150$ & 48.30 & 596.00 \\
\hline $646 \mathrm{~B}-5 \mathrm{H}-3,0$ & 39.00 & 458.61 \\
\hline $646 \mathrm{~B}-5 \mathrm{H}-3,30$ & 39.30 & 463.47 \\
\hline $646 \mathrm{~A}-6 \mathrm{H}-1,0$ & 50.00 & 610.00 \\
\hline $646 \mathrm{~A}-6 \mathrm{H}-6,150$ & 59.00 & 694.33 \\
\hline $646 \mathrm{~B}-6 \mathrm{H}-4,30$ & 48.81 & 600.00 \\
\hline 646B-6H-6, 150 & 53.01 & 637.24 \\
\hline $646 \mathrm{~A}-7 \mathrm{H}-1,0$ & 59.60 & 699.11 \\
\hline $646 \mathrm{~A}-7 \mathrm{H}-7,60$ & 69.20 & 790.79 \\
\hline $646 \mathrm{~A}-8 \mathrm{H}-1,0$ & 69.30 & 791.64 \\
\hline $646 \mathrm{~A}-8 \mathrm{H}-4,120$ & 75.00 & 840.00 \\
\hline
\end{tabular}

given in Appendix A. Mass magnetic susceptibility $(X)$ is proportional to the concentration of magnetic minerals per unit sediment mass. $X$ usually reflects changes in terrigenous flux and pelagic dilution (e.g., biogenic $\mathrm{CaCO}_{3}$ ). We also express magnetic mineral concentration as an accumulation rate using the equation:

$$
K_{a r}=k \times S,
$$

where $K_{a r}$ represents the magnetic mineral accumulation rate and $k$ is the volume magnetic susceptibility. The volume of each sample is $5 \mathrm{~cm}^{3}$.

$X_{A R M}$ is the magnetic anhysteretic susceptibility, which is also proportional to the concentration of magnetic minerals per unit mass; however, this value is more grain-size dependent than $X$. The ratio $X_{A R M} / X$ expresses relative variations of particle size of magnetic minerals. The larger the $X_{A R M} / X$, the smaller the particle size; we interpret low values of $X_{A R M} / X$ at Site 646 as reflecting the winnowing of the finer-grained sediment fractions by stronger bottom currents.

\section{$\mathrm{CaCO}_{3}$ and Planktonic Foraminifer Accumulation Rates}

Carbonate concentration, expressed as percent of $\mathrm{CaCO}_{3}$ was determined by the coulometric technique (Huffman, 1977) (Appendix B). Ninety-eight of the samples used for carbonate analyses also were used for rock-magnetic measurements.

The percent of $\mathrm{CaCO}_{3}$ was converted to carbonate accumulation rate by the equation:

$$
\mathrm{CaCO}_{3 a r}=\left(\% \mathrm{CaCO}_{3} / 100\right) \times B A R,
$$

where $\mathrm{CaCO}_{3 a r}$ is the carbonate accumulation rate.

Planktonic foraminiferal concentration (Aksu et al., this volume) is expressed as numbers per gram and converted to planktonic foraminifer accumulation rate $\left(P f_{a r}\right)$ by multiplying by $B A R$. We used these two parameters to indicate pelagic flux.

\section{Statistical Analyses}

Cross-correlation analyses were used to determine the degree of correlation and the lag time between the various parameters and oxygenisotope time series. Spectral analyses (Fast Fourier Transform [FFT] using Bloomfield's [1976] computer program) were applied to the rockmagnetic and accumulation-rate time series to detect the concentration
Table 2. Depths of oxygen-isotope events

\begin{tabular}{|c|c|c|}
\hline Event & $\begin{array}{l}\text { Depth } \\
\text { (mbsf) }\end{array}$ & $\begin{array}{c}\text { Age } \\
\text { (k.y.) }\end{array}$ \\
\hline 1.00 & 0 & \\
\hline 1.10 & 1.00 & \\
\hline 2.20 & 2.50 & 1 \\
\hline 3.10 & 3.40 & \\
\hline 3.30 & 5.30 & \\
\hline 4.20 & 7.53 & \\
\hline 5.30 & 9.20 & \\
\hline 5.50 & 10.01 & 12 \\
\hline 6.20 & 12.10 & 13 \\
\hline 6.50 & 15.40 & 17 \\
\hline 6.60 & 16.30 & 18 \\
\hline 7.10 & 16.60 & 19 \\
\hline 7.30 & 17.30 & 21 \\
\hline 7.40 & 19.60 & 22 \\
\hline 7.50 & 20.50 & 23 \\
\hline 8.20 & 22.30 & 24 \\
\hline 8.30 & 23.70 & 25 \\
\hline 8.40 & 24.71 & 26 \\
\hline 8.50 & 25.99 & 28 \\
\hline 8.60 & 26.35 & 29 \\
\hline 9.10 & 27.13 & 31 \\
\hline 9.20 & 27.89 & 32 \\
\hline 9.30 & 29.00 & 33 \\
\hline 10.20 & 29.90 & 34 \\
\hline 11.10 & 32.70 & 36 \\
\hline 11.30 & 33.92 & 40 \\
\hline 11.40 & 35.90 & 41 \\
\hline 12.10 & 36.30 & 42 \\
\hline 12.20 & 37.48 & 43 \\
\hline 12.31 & 38.24 & 44 \\
\hline 13.12 & 41.00 & 49 \\
\hline 13.20 & 43.10 & 51 \\
\hline 14.20 & 45.70 & 53 \\
\hline 14.30 & 45.90 & 55 \\
\hline 15.10 & 47.10 & 57 \\
\hline 15.30 & 48.30 & 59 \\
\hline 15.50 & 50.85 & 61 \\
\hline 16.22 & 52.10 & 62 \\
\hline 17.10 & 56.04 & 66 \\
\hline 18.22 & 59.30 & 69 \\
\hline 18.30 & 60.20 & 71 \\
\hline 19.10 & 64.14 & 73 \\
\hline 20.23 & 65.15 & 75 \\
\hline 21.10 & 66.00 & 77 \\
\hline 21.30 & 68.40 & 78 \\
\hline
\end{tabular}
from Site 646.

of variance at earth orbital frequencies (Hays et al., 1976). For this report, we concentrate only on those periodicities that result from well-established orbital parameters: precession (18 and 23 k.y.), obliquity (41 k.y.), and eccentricity (100 and 413 k.y.). Both unsmoothed and smoothed (using modified Daniell smoothing [Bloomfield, 1976]) curves of spectral density are given. The spectral routine we used cannot eliminate spurious effects, such as side-lobe leakage or ringing; however, these can be reduced by smoothing. To test the reliability of the spectral routine, we included analysis of the SPECMAP oxygen-isotope data, the spectral characteristics of which are well known (Imbrie et al., 1982).

\section{RESULTS}

Figure 2 shows the location of tie-points between the Site 646 oxygen- isotope record and the SPECMAP master curve, which is an indicator of variations in global ice volume. Oxygen-isotope ratios from planktonic foraminfers reflect not only changes in ice volume, but also more local changes in surface-water salinity and temperature. However, despite this potential source of error, the good correlation between the oxygen-isotope data, and the SPECMAP master curve (correlation coefficient $=$ 0.811 ; Table 3) suggests to us that good time control has been achieved. The resulting age-depth model is shown in Figure 3.

Variations in sedimentation rate and $K_{a r}$ are shown in Figure 4. Sedimentation rate lags behind the oxygen-isotope record by 


\section{OXYGEN ISOTOPES}

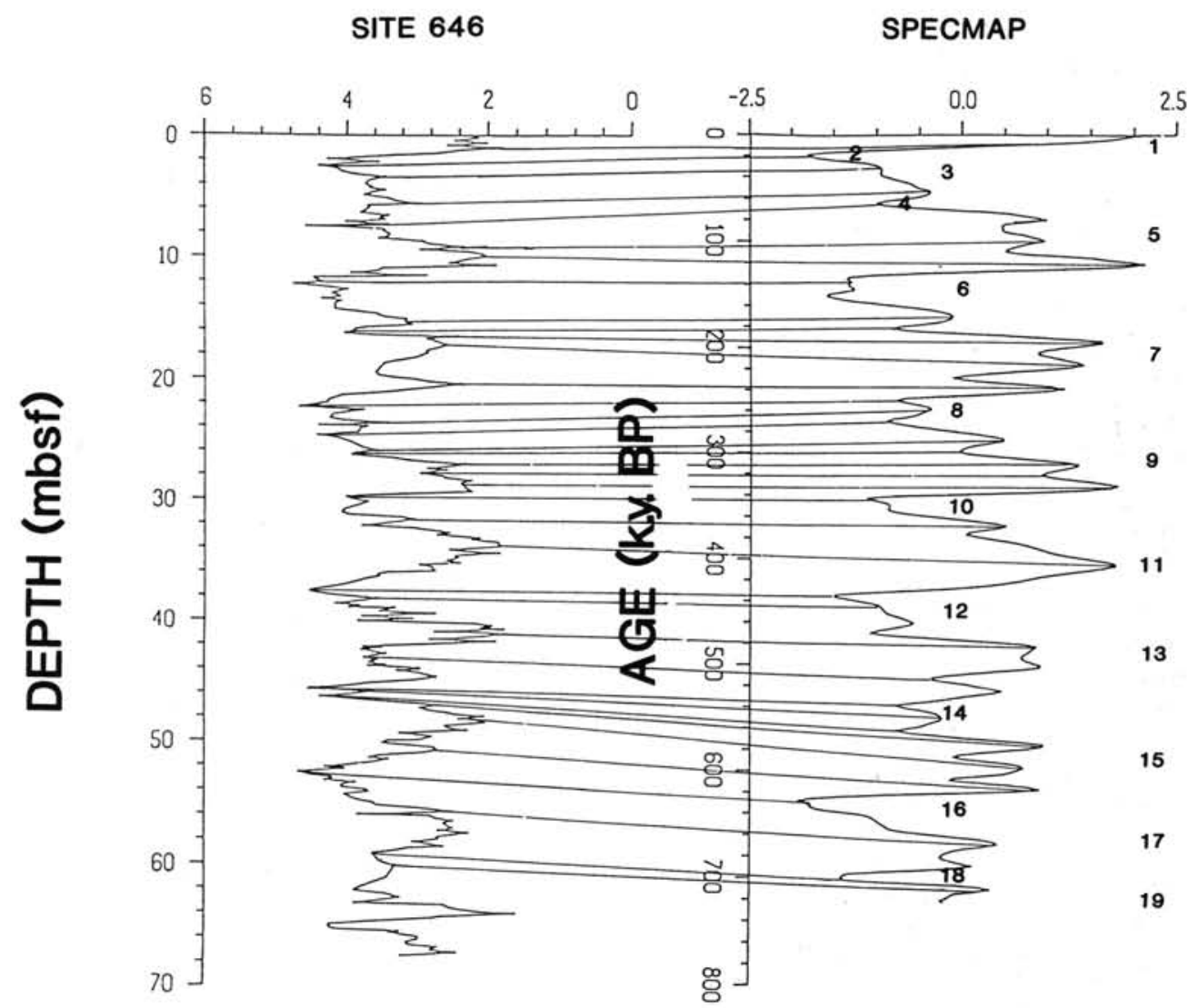

Figure 2. Comparison of Site 646 oxygen-isotope record and the SPECMAP master curve. Tie-points shown are also given in Table 1 . Numbers on the right side of the figure are the oxygen-isotope stages.

4 k.y. from oxygen-isotope stage 21 to stage 11 , and 18 k.y. from stage 11 to stage 1 (Table 3 ). $K_{a r}$ mimics the sedimentationrate curve.

A change in character of sedimentation occurred approximately 400 k.y. BP. This change in sedimentation is suggested by changes in physical properties, rock-magnetic parameters, and accumulation rates $\left(\left[\mathrm{CaCO}_{3}\right]_{a r}\right.$ and $\left.P f_{a r}\right)$. When plotted as a function of depth, bulk density (porosity) increases (decreases) monotonically (Srivastava, Arthur, et al., 1987). However, when plotted as a function of time, bulk density and porosity vary in a cyclic manner downsite, having lower and higher values, respectively, from 400 k.y. to 0 k.y. than from 782 k.y. to 400 k.y. (Fig. 5). Because there is no significant change in bulk sediment grain size or mineralogy (Dadey, pers. comm., 1988), we suggest that the distinct change in physical properties at approximately 400 k.y. probably results from changes in the character of sedimentation.

$X$ and $X_{A R M} / X$ (Fig. 6) do not lag the SPECMAP record from oxygen-isotope stages 21 to 11, but lag the SPECMAP record by 8 k.y. and 14 k.y., respectively, from stages 11 to 1 . $\mathrm{CaCO}_{3 a r}$ and $P f_{a r}$ (Fig. 7) do not lag the SPECMAP record from the oxygen-isotope stages 21 to 11 curve, but lag by 6 k.y. and $8 \mathrm{k} . \mathrm{y}$. respectively, from oxygen-isotope stages 11 to 1 (Table 3). Thus, the parameters we discuss here are in phase (i.e., 0 k.y. lag) with changes in ice volume from stages 21 to 11 , and are out of phase from stages 11 to 1 . We separated the sequence into
Table 3: Results of cross-correlation analyses of parameters for samples from Site 646 discussed in the text and the SPECMAP oxygen-isotope curve.

\begin{tabular}{lrrr}
\hline \multicolumn{1}{c}{ Parameter } & $\begin{array}{c}\text { Age sequence } \\
(\mathrm{k} . \mathrm{y} .)\end{array}$ & $\begin{array}{c}\text { Correlation } \\
\text { coefficient }\end{array}$ & $\begin{array}{c}\text { Lag } \\
(\mathrm{k} . \mathrm{y} .)\end{array}$ \\
\hline Age-depth & & 0.999 & \\
Oxygen data-SPECMAP & & 0.811 & \\
Sedimentation rate-SPECMAP & $0-780$ & 0.232 & 10 \\
& $0-394$ & 0.313 & 18 \\
& $394-780$ & 0.348 & 4 \\
$X_{\text {ARM }} / X$-SPECMAP & $0-782$ & -0.395 & 10 \\
& $0-392$ & -0.504 & 14 \\
& $392-782$ & -0.304 & 0 \\
$X$-SPECMAP & $0-782$ & 0.176 & 8 \\
& $0-392$ & 0.316 & 8 \\
& $392-782$ & 0.103 & 0 \\
$K_{a r}$-SPECMAP & $0-780$ & 0.232 & 10 \\
& $0-392$ & 0.313 & 18 \\
& $392-780$ & 0.339 & 6 \\
f $_{a r}$-SPECMAP & $0-782$ & -0.203 & 8 \\
& $0-392$ & -0.282 & 8 \\
$\mathrm{CACO}_{3 a r}$-SPECMAP & $392-782$ & 0.055 & 0 \\
& & & \\
& $0-782$ & 0.219 & 0 \\
& $0-392$ & -0.093 & 8 \\
& $392-782$ & 0.528 & 0 \\
\hline
\end{tabular}




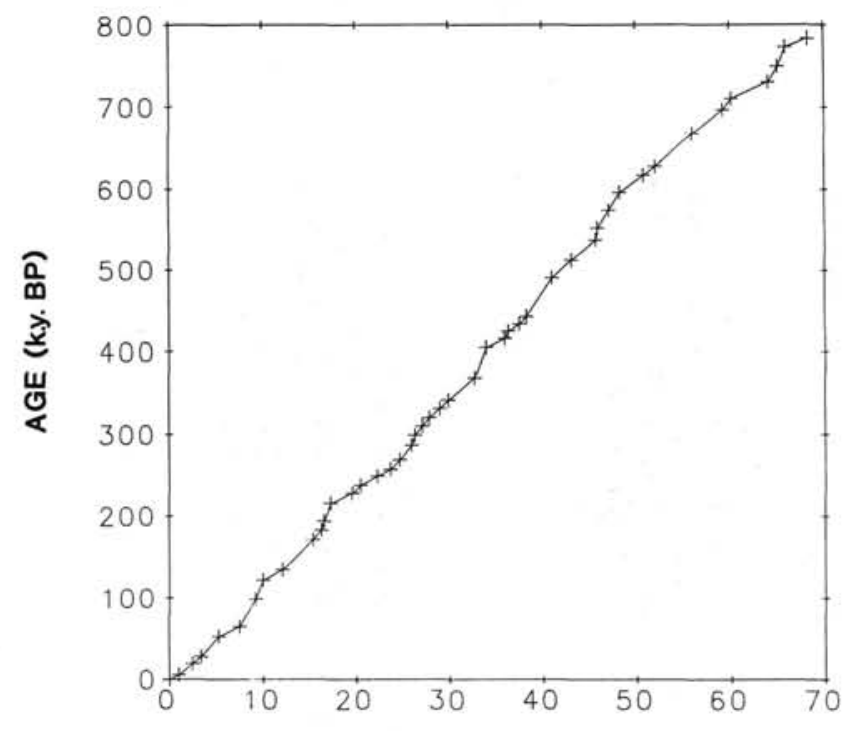

DEPTH (mbsf)

Figure 3. Age vs. depth model for Site 646, determined from 45 tiepoints.

two time series for spectral analysis: 782-392 k.y. and 392-0 k.y. because of the observed phase shift.

\section{Spectral Analyses}

Spectral analysis of the SPECMAP oxygen-isotope data (Fig. 8) shows a dominant periodicity of $102 \mathrm{k} . \mathrm{y}$., which is within the eccentricity band, at about 100 k.y.

Spectral analyses of $X$ (Fig. 9) reflect both short- and longperiod variations in orbital parameters. In the 782 to 392 k.y. time series, the obliquity signal is much stronger than the precessional signal. However, a periodicity close to the precessional band is seen as a significant component from 392-0 k.y. The eccentricity cycle is prominent during both time slices.

Spectral analyses of $K_{a r}$ and sedimentation rate show the same periodicities. Thus, we present only the spectral analyses of $K_{a r}$ to represent both. $K_{a r}$ (Fig. 10) reflects the same dominant periodicity in both the upper and lower portions of the time series: eccentricity.

The spectral pattern of $X_{A R M} / X$ from 782 to $392 \mathrm{k} . \mathrm{y}$. is dominated by a nominal 73-k.y. periodicity. However, the spectral pattern of $X_{A R M} / X$ (Fig. 11) is dominated by about a $100-\mathrm{k} . \mathrm{y}$. cycle in the upper 392 k.y.

The spectral analyses of $P f_{a r}$ and $\left(\mathrm{CaCO}_{3}\right)_{a r}$ (Figs. 12 and 13) show periodicities related to obliquity and precession from stages 21 to 11 , and periodicities within the eccentricity and precessional bands from stages 11 to 1 .

\section{DISCUSSION}

The shift in parameters at approximately 400 k.y. BP suggests an overall change in sedimentation style. From oxygen-isotope stages 21 to 11 , the differences in lag between sedimentation-rate and accumulation-rate parameters $\left(P f_{a r}\right.$ and $\left.\mathrm{CaCO}_{3 a r}\right)$ and $X$ and $K_{a r}$ are negligible. All of the parameters are in phase with changes in ice volume from oxygen-isotope stages 11 to 21 .

From oxygen-isotope stages 11 to 1 , there is a significant difference in the lags of sedimentation-rate (about 18 k.y.) and accumulation-rate parameters $\left(P f_{a r}\right.$ and $\left.\left[\mathrm{CaCO}_{3}\right]_{a r}\right)$ (about 8 k.y.) relative to the SPECMAP oxygen-isotope curve. This lag difference is also seen with $X$ (about 8 k.y.) and $K_{a r}$ (about 18 k.y.).
On the basis of the comparable lag differences, we might expect to see a direct relationship between the amount of carbonate and magnetic mineral concentration. Unlike Robinson (1986) and Bloemendal et al. (1988), we found no significant negative correlation between percentage of $\mathrm{CaCO}_{3}$ and susceptibility (Fig. 14). However, the carbonate fraction at Site 646 is composed of both biogenic and detrital components (Srivastava, Arthur, et al., 1987), and our analytical methods cannot differentiate between the two types of carbonate. $X$ is partly determined by the concentration of detrital carbonate and will be further diluted by the addition of biogenic carbonate. The effect of sedimentation of carbonate from different sources and processes is to mask a direct relationship between carbonate and magnetic mineral concentration.

The change in lag time for $K_{a r}$ and $X_{A R M} / X$ relative to the SPECMAP record suggests that whereas the period of bottomcurrent activity remains the same throughout the record, its phase with respect to ice volume changes at approximately $400 \mathrm{k} . \mathrm{y}$. BP. The negative correlation coefficient between $X_{A R M} / X$ and oxygen isotopes further suggests that finer-grained magnetic minerals are deposited during glacial periods. This result is the opposite of that found by Robinson (1986) and Bloemendal et al. (1988). Robinson concluded that the pattern of magnetic mineral grain-size changes observed at his sites largely resulted from the deposition of coarse-grained magnetic minerals during glacials via ice rafting. Bloemendal et al. concluded that their magnetic grain-size changes resulted from eolian transport of relatively coarse-grained minerals during glacials in combination with a flux of fine-grained magnetic material during interglacials of either fluvial or in-situ biogenic origin. At Site 646, we interpret $X_{A R M} / X$ variations to reflect changes in intensity of bottom currents, which are negligible or nonexistent during glacials (Ruddiman and McIntyre, 1981). Therefore, minima in bottom-current activity occur in phase with glacial maxima from oxygen-isotope stages 21 to 11 , whereas they lag behind glacial maxima by 14 k.y. from oxygen-isotope stages 11 to 1 .

Spectral analyses suggest that the obliquity signal (about 41 k.y.) is significant for controlling planktonic foraminiferal flux and magnetic mineral concentration and flux from stages 21 to 11 , but it is insignificant from stages 11 to 1 (Figs. 9 and 11). Thus, we attribute the change in the style of sedimentation observed at Site 646 to the decreasing influence of the obliquity forced environmental parameters at about $400 \mathrm{k} . \mathrm{y}$.

We cannot explain all of the peaks we see in our spectral analyses. However, the 34-k.y. periodicity of $\mathrm{CaCO}_{3 \text { ar }}$ found from oxygen-isotope stage 11 to oxygen-isotope stage 1 is near the 31-k.y. periodicity reported by Pisias and Rea (1988) for seasurface temperatures of the central equatorial Pacific. Pisias and Rea suggested that this signal may be real, and possibly a reinforcement of the precessional and obliquity signals. The cause of the 73-k.y. periodicity of $X_{A R M} / X$ from oxygen-isotope stage 21 to oxygen-isotope stage 11 is unknown. However, the 73-k.y. periodicity is near the difference in tone between obliquity and eccentricity: $1 / 41-1 / 100=1 / 69$.

A change in the significance of orbital frequencies for oxygen isotopes has been noted previously to occur near the stage $22 / 21$ boundary, when the dominant signal changes from obliquity to eccentricity (Williams et al., 1981). Ruddiman et al. (1986) attributed this shift to the influence of the uplift of the Sierra Nevada mountain range and the Himalyas. These uplifts during the late Pliocene-early Pleistocene could have resulted in a more meridional upper atmospheric circulation that would bring polar air farther south, thus quickening the pace of glacial growth (Ruddiman et al., 1986).

Jansen et al. (1986) reported evidence of a long-term 350 to $400 \mathrm{k} . \mathrm{y}$. cycle in sedimentation patterns on a global basis. They attributed this long-term change to the about 413-k.y. periodic- 
SEDIMENTATION RATE (m/my.)

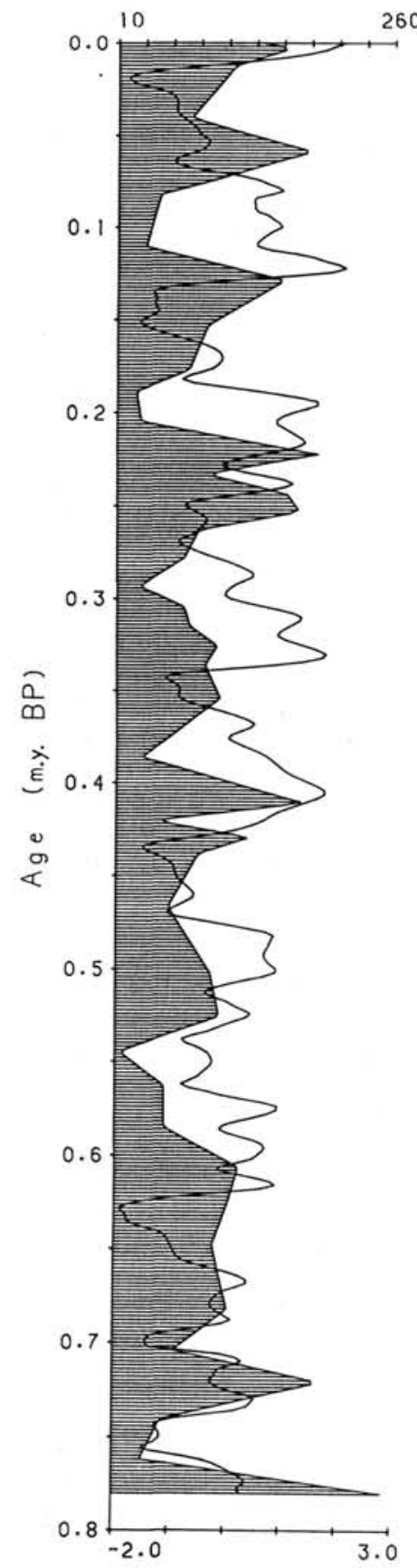

Kar

0

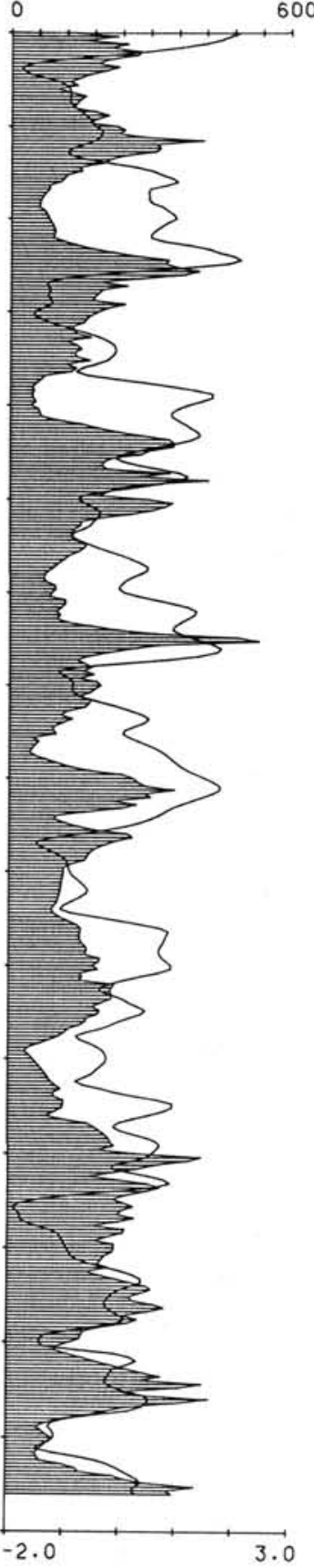

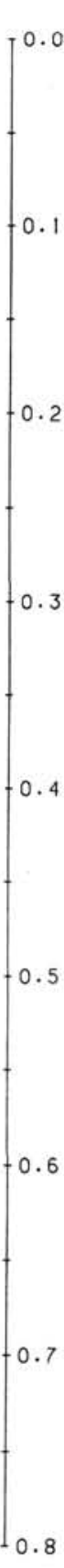

Figure 4. Sedimentation rate and $K_{a r}$ vs. age.

\section{BULK DENSITY $\left(\mathrm{g} / \mathrm{km}^{3}\right)$}

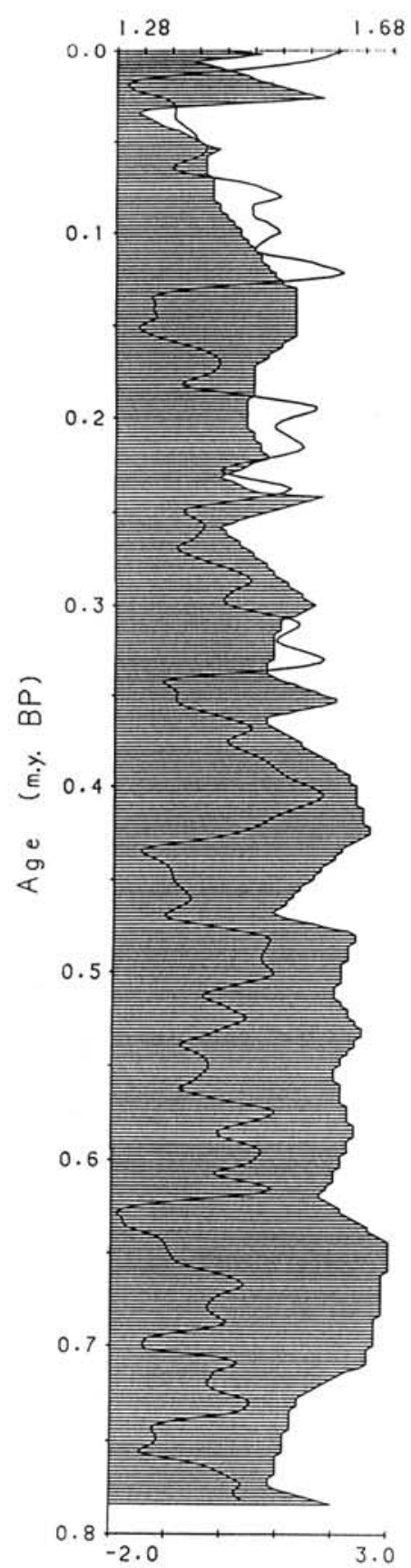

POROSITY

(\%)

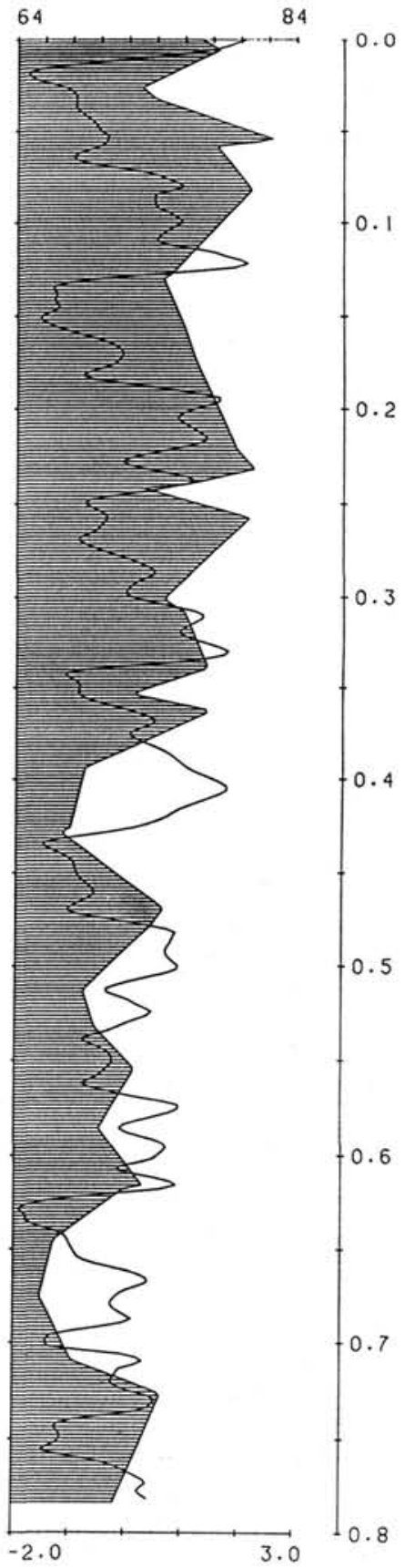

Figure 5. Bulk density and porosity vs. age.

1. The change in lag time with respect to the SPECMAP oxygen-isotope curve of $X_{A R M} / X$ (used to indicate relative changes in bottom-current intensity) and $K_{a r}$ (used as an indicator of terrigenous flux) from 0 k.y. for both parameters, during stages 21 to 11 , to 14 and $18 \mathrm{k} . y$., respectively, during stages 11 to 1 .

2. The change in lag time, with respect to the SPECMAP oxygen-isotope curve, of magnetic mineral concentration $(X)$, $\mathrm{CaCO}_{3 a r}$, and $P f_{a r}$, used as indicators of pelagic flux, from 0 k.y. during stages 21 to 11 to $8 \mathrm{k}$.y. during stages 11 to 1 for each parameter. 
3. Reduced power of the about 41-k.y. obliquity signal in the $P f_{a r}, K_{a r}$, and $X$ records during stages 11 to 1 .

\section{ACKNOWLEDGMENTS}

The University of Rhode Island authors were supported by JOI USSAC P.O. Nos. 76322 and 70760 for Leg 105. A. E. Aksu was supported by NSERC Grant A6896. We thank Sheila Rieg for her assistance in analytical work and Mark Wimbush for his assistance with interpreting the spectral results. We thank Paula Weiss, Alan Spies, and Jeffrey Corbin for assistance during sampling at the ODP East Coast Repository.

\section{REFERENCES}

Bloemendal, J., Lamb, B., and King, J. W.,1988. Paleoenvironmental implications of rock-magnetic properties of late Quaternary deepsea sediments from the eastern equatorial Atlantic. Paleoceanography, 3:61-88.

Hays, J. D., Imbrie, J., and Shackleton, N. J.,1976. Variations in the earth's orbit: pacemaker of the ice ages. Science, 194:1121-1132.

Huffman, E.W.D., 1977. Performance of a new automatic carbon dioxide coulometer. Microchem. J., 22:567-573.

Imbrie, J., Hays, J. D., Martinson, D. G., McIntyre, A., Mix, A. C., Morley, J. J., Pisias, N. G., Prell, W. G., and Shackleton, N. J., 1984. The orbital theory of Pleistocene climate: support from a revised chronology of the marine oxygen-isotope record. In Berger et al. (Eds.), Milankovitch and Climate: New York (NATO), 269-306.

Jansen, J.H.F., Kuijpers, A., and Troelstra, S. R., 1986. A mid-Brunhes climatic event: long-term changes in global atmospheric and ocean circulation. Science, 232:619-622.

Kirschvink, J. L., and Chang, S-B. R., 1984. Ultrafine-grained magnetite in deep-sea sediments: possible bacterial microfossils. Geology, $12: 559-562$.
Petersen, N., von Dobeneck, T., and Vali, H., 1986. Fossil bacterial magnetite in deep-sea sediments from the South Atlantic Ocean. $\mathrm{Na}$ ture, 320:611-615.

Pisias, N. G., and Rea, D. K., 1988. Late Pleistocene paleoclimatology of the central equatorial Pacific: sea-surface response to the southeast trade winds. Paleoceanography, 3:21-38.

Prell, W. L., Imbrie, J., Martinson, D. G., Morley, J. J., Pisias, N. G., Shackleton, N. J., and Streeter, H. F., 1987. Graphic correlation of oxygen isotope stratigraphy application to the late Quaternary. Paleoceanography, 1:137-162.

Robinson, S., 1986. The late Pleistocene paleoclimatic record of North Atlantic deep-sea sediments revealed by mineral-magnetic measurements. Phys. Earth Planet. Interiors, 42:22-47.

Ruddiman, W. F., and McIntyre, A., 1979. Warmth of the subpolar North Atlantic Ocean during Northern Hemisphere ice-sheet growth. Science, 204:173-175.

1981. The North Atlantic during the last deglaciation. Palaeogeogr., Palaeoclimatol., Palaeoecol., 35:145-214.

Ruddiman, W. F., Raymo, M., and McIntyre, A., 1986. Matuyama 41,000-year cycles: North Atlantic Ocean and Northern Hemisphere ice sheets. Earth Planet. Sci. Lett., 80:117-129.

Srivastava, S. P., Arthur, M., et al., 1987. Proc. ODP, Init. Repts, 105: College Station, TX (Ocean Drilling Program).

Williams, D. F., Moore, W. S., and Fillon, R. H., 1981. Role of glacial Arctic Ocean ice sheets in Pleistocene oxygen isotope records and sea level records. Earth Planet. Sci. Lett., 56:157-166.

Date of initial receipt: 6 April 1988

Date of acceptance: 29 September 1988

Ms 105B-177 


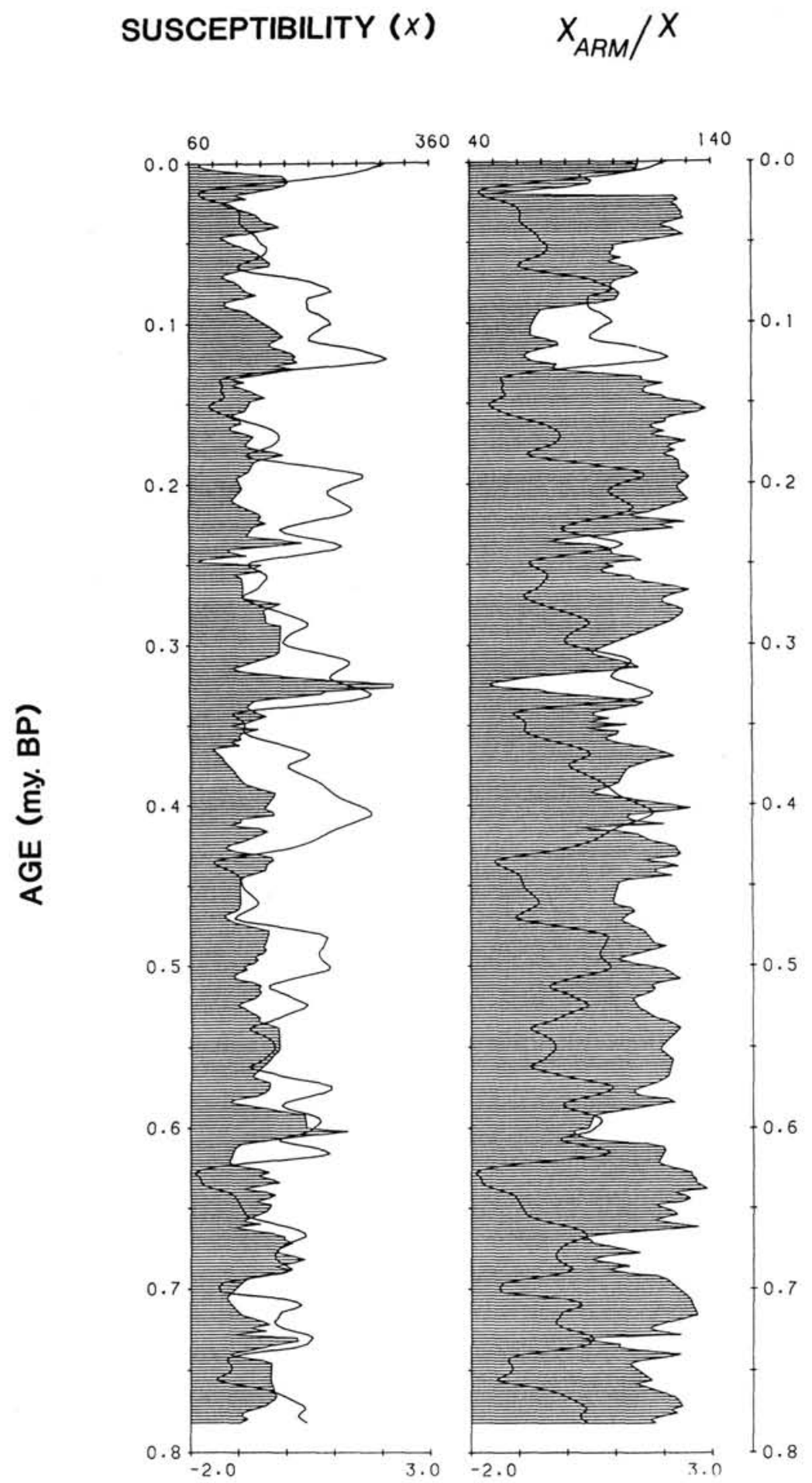

Figure 6. $X$ and $X_{A R M} / X$ vs. age. 

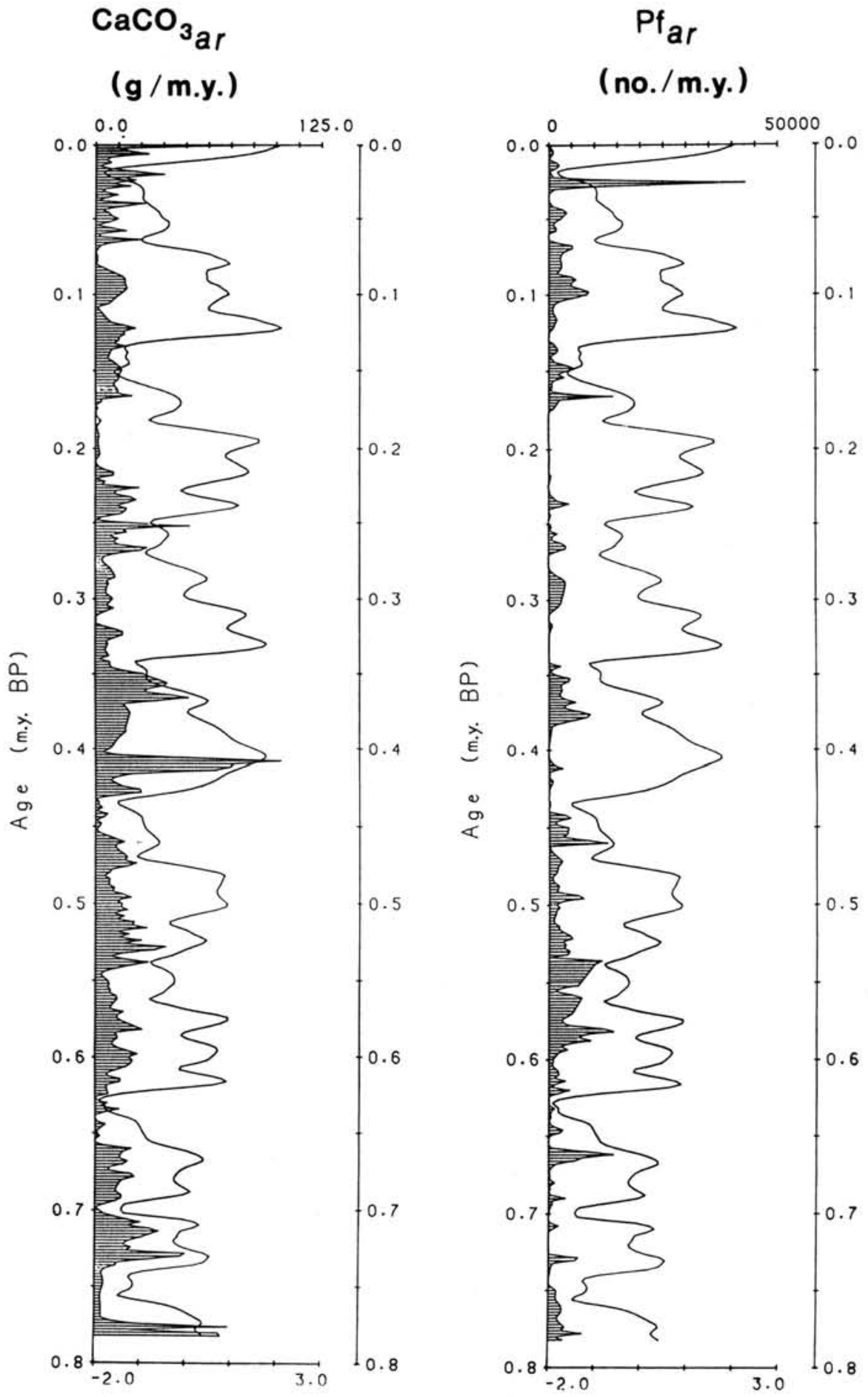

Figure 7. $\mathrm{CaCO}_{3 a r}$ and $P f_{a r}$ vs. age. 
F. R. HALL ET AL.

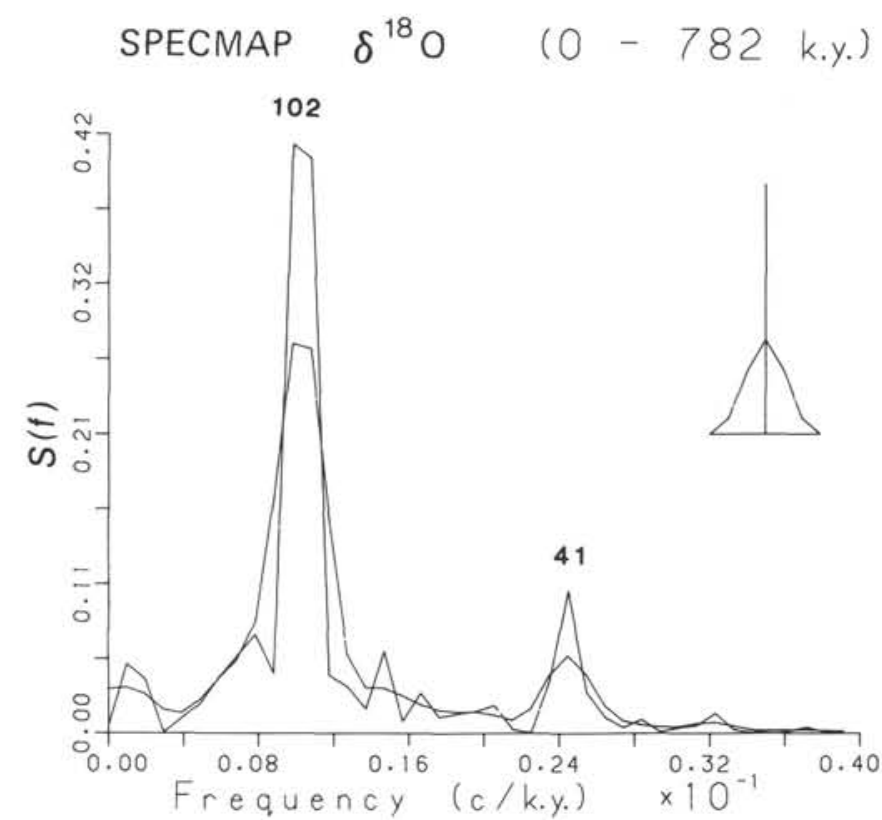

Figure 8. Spectral analyses of SPECMAP master oxygen-isotope curve. Numbers in figure represent periodicity in thousand years. The bell to the right represents the size of the Daniell smoothing window.
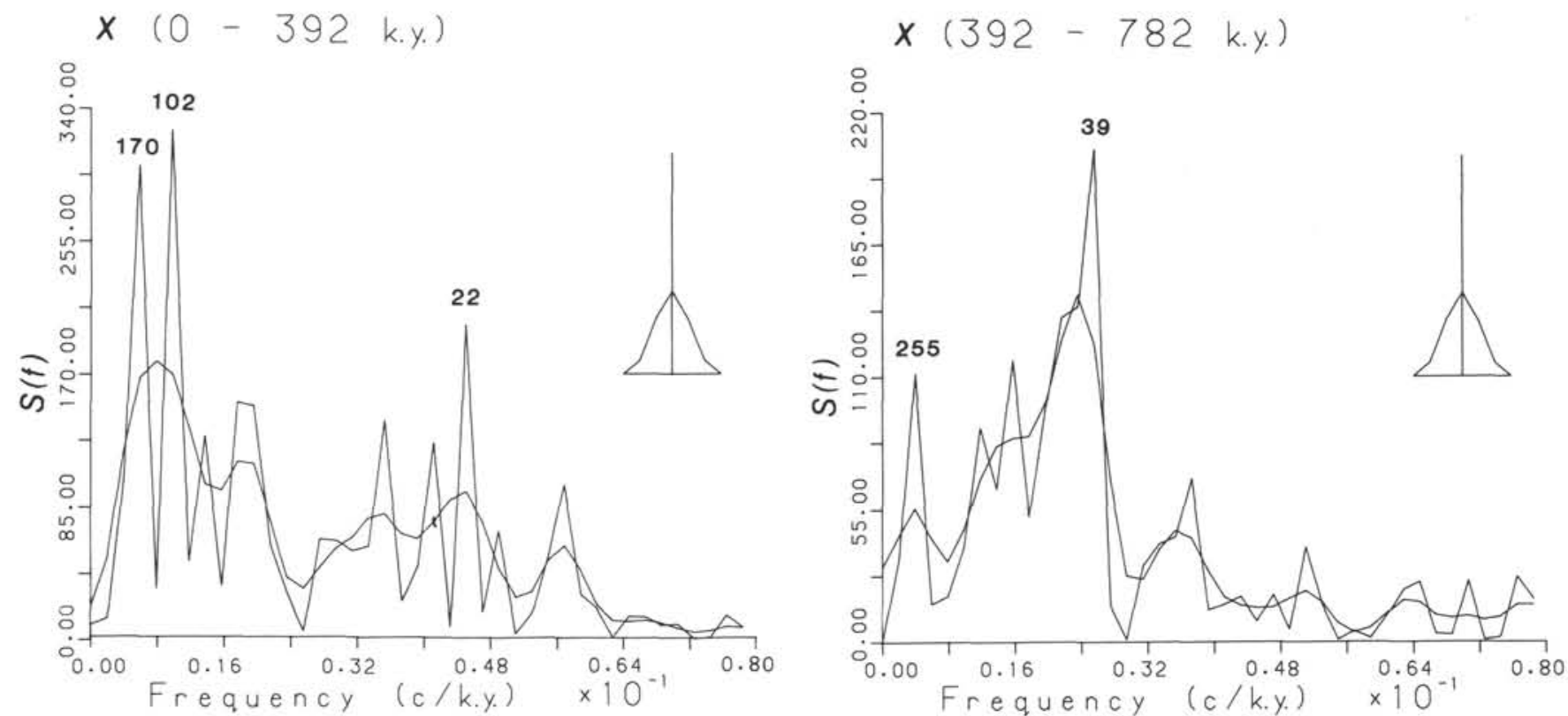

Figure 9. Spectral analyses of data discussed in text. 

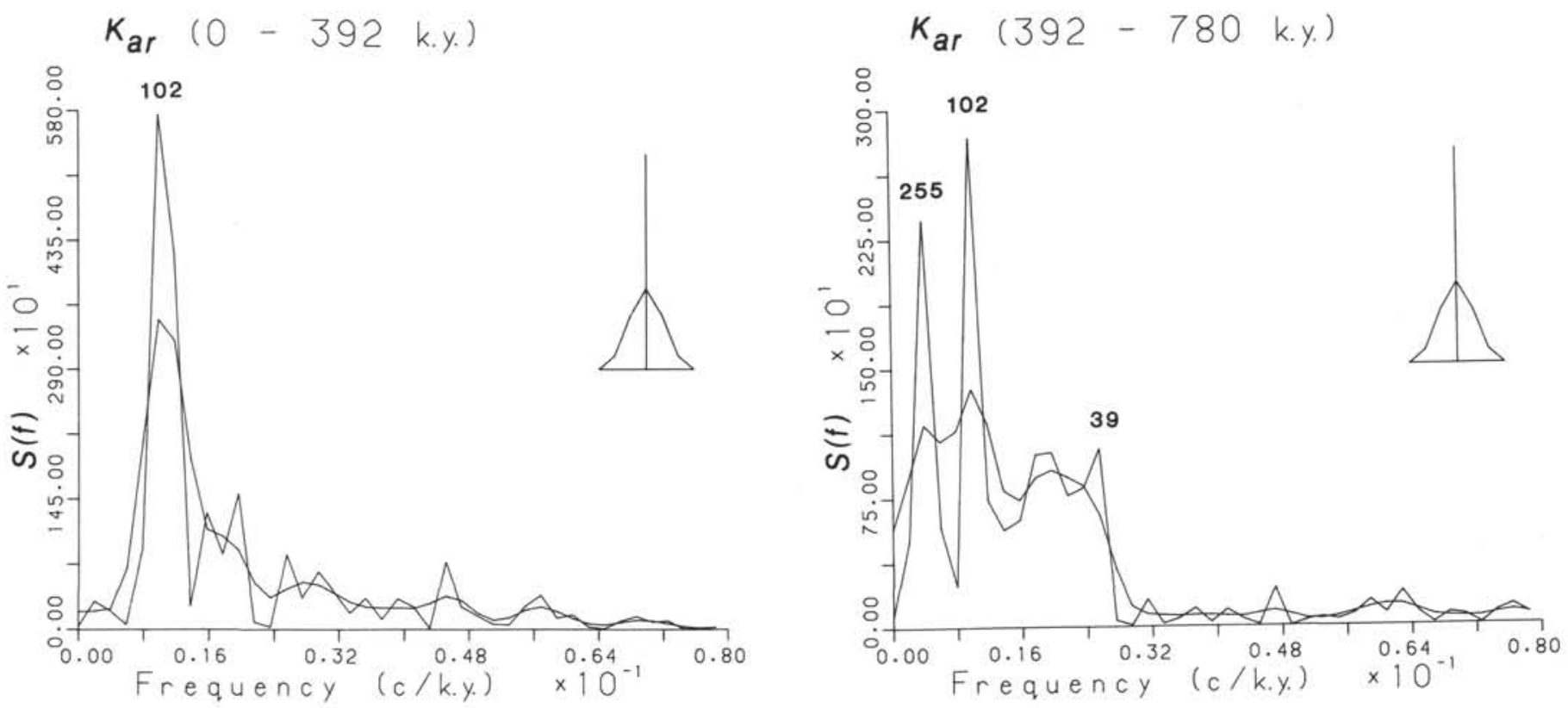

Figure 10. Spectral analyses of data discussed in text.
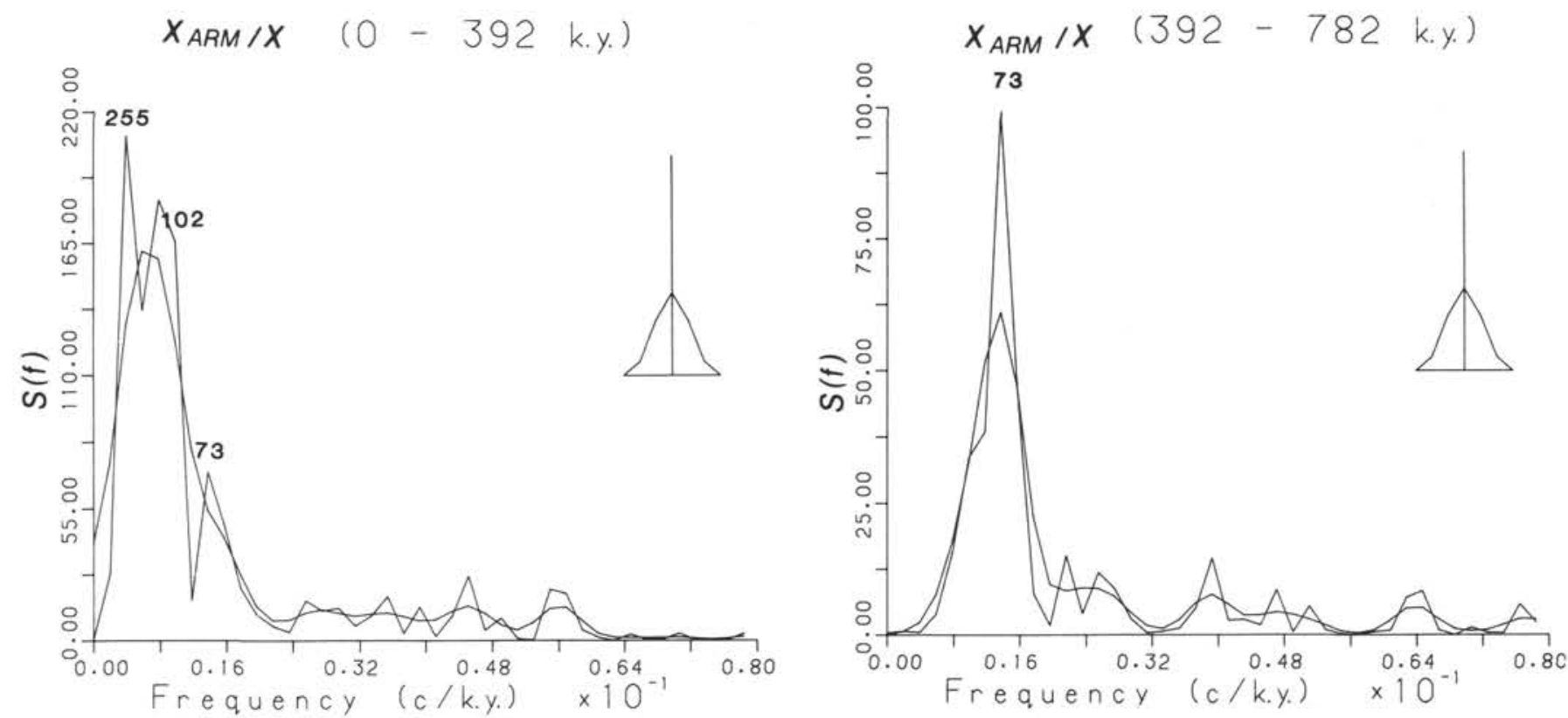

Figure 11. Spectral analyses of data discussed in text. 

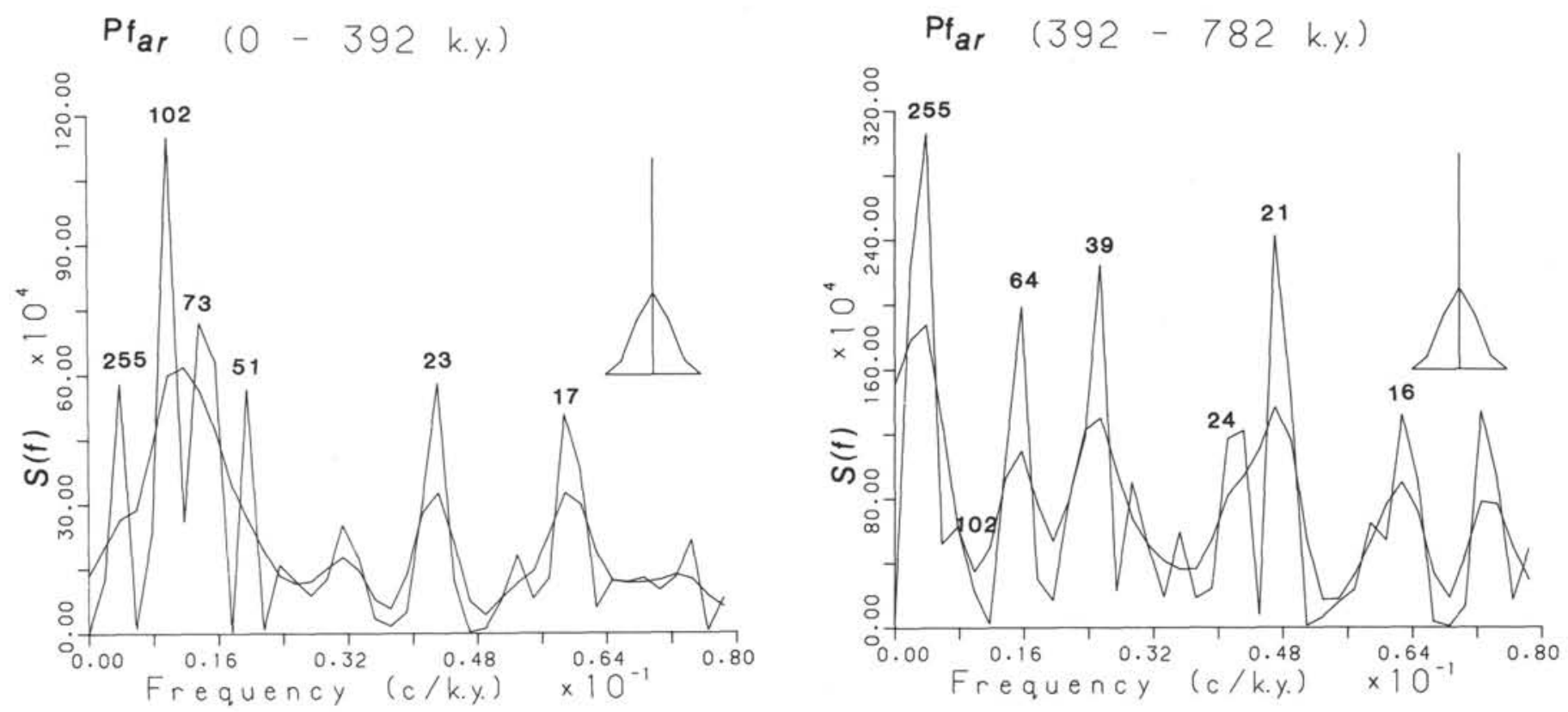

Figure 12. Spectral analyses of data discussed in text.
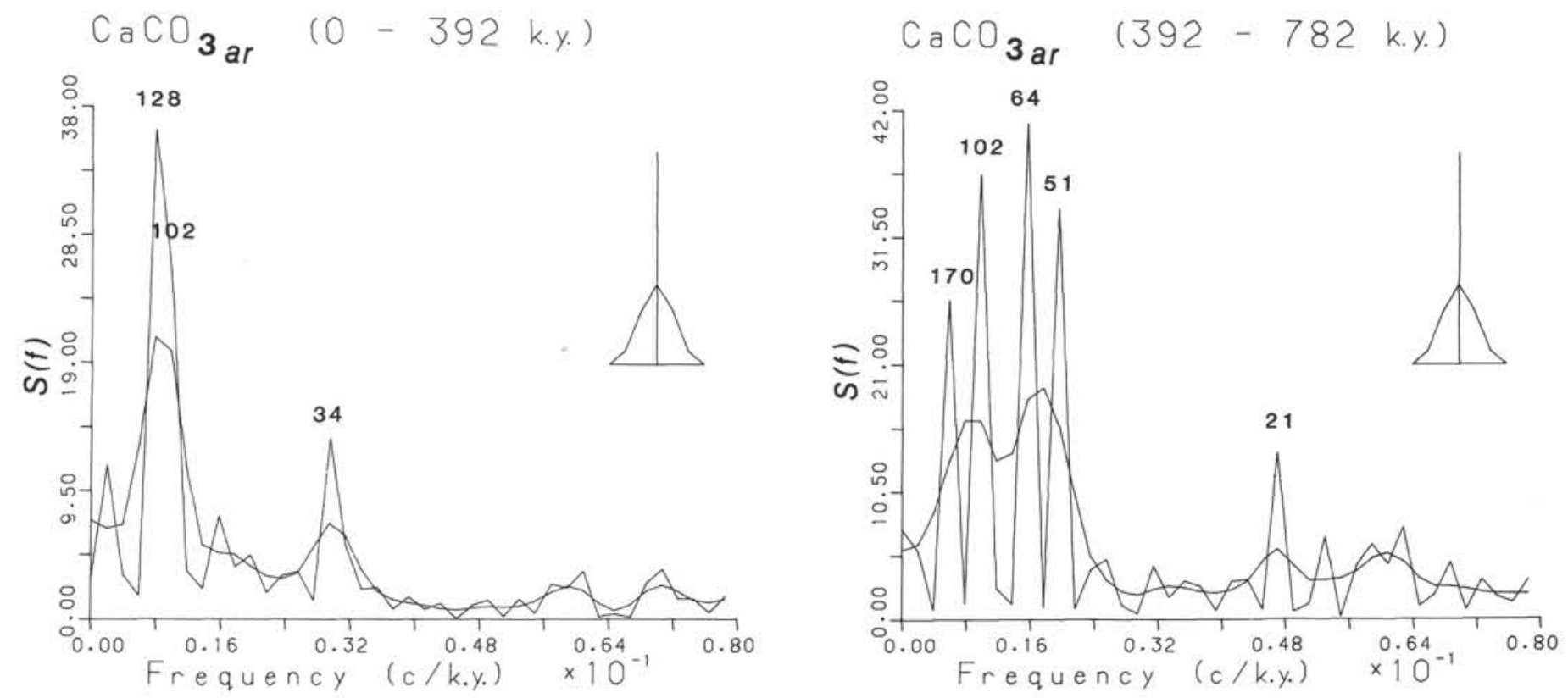

Figure 13. Spectral analyses of data discussed in text. 
MIDDLE TO LATE QUATERNARY SEDIMENT FLUXES

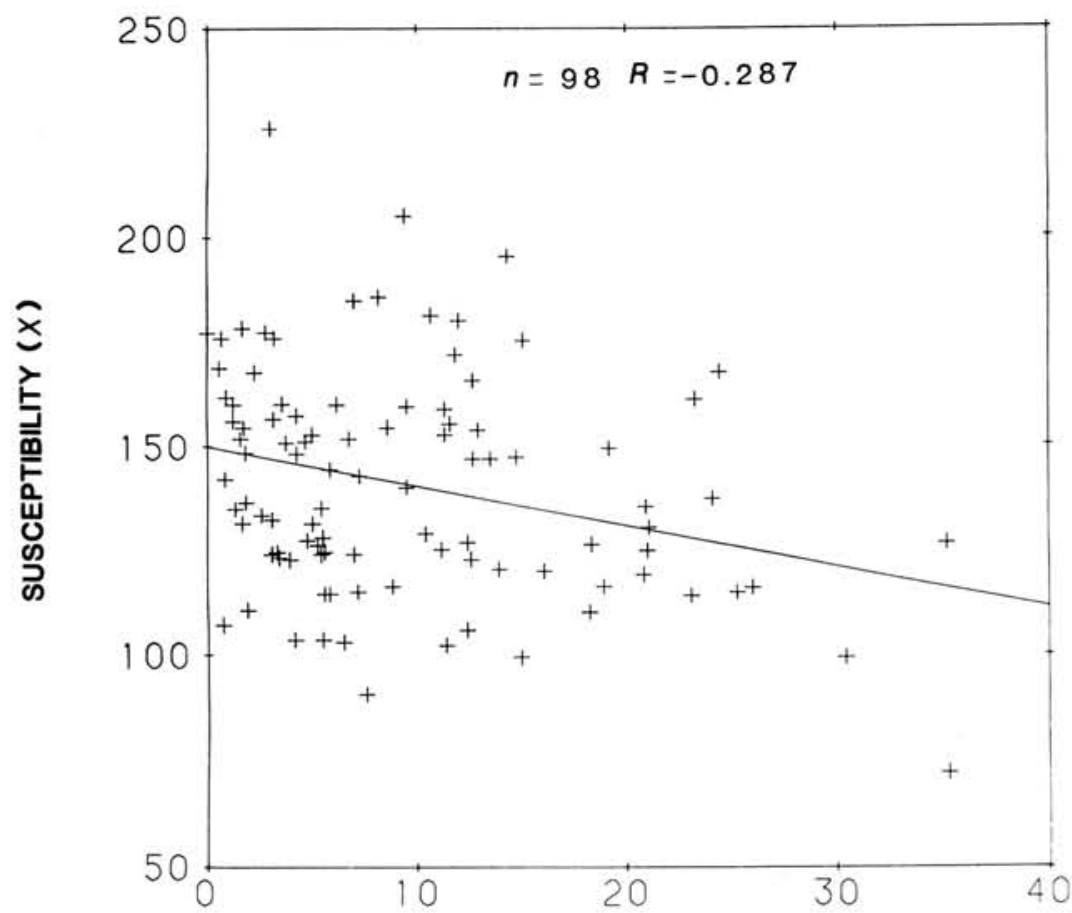

Percentage of $\mathrm{CaCO}_{3}$

Figure 14: $X$ vs. percentage of $\mathrm{CaCO}_{3}$. 
Appendix A

Percentages of $\mathrm{CaCO}_{3}$, TOC, Nitrogen, and $\mathrm{C} / \mathrm{N}$ ratio for Site 646 .

\begin{tabular}{|c|c|c|c|c|c|c|c|c|}
\hline \multicolumn{4}{|c|}{$\begin{array}{l}\text { Core, section } \\
\text { depth }(\mathrm{cm})\end{array}$} & \multirow{2}{*}{$\begin{array}{l}\begin{array}{l}\text { Depth } \\
\text { (mbsf) }\end{array} \\
0.00\end{array}$} & \multirow{2}{*}{$\begin{array}{c}\begin{array}{c}\mathrm{CaCO}_{3} \\
(\%)\end{array} \\
35.25\end{array}$} & \multirow[t]{2}{*}{$\begin{array}{l}\text { TOC } \\
(\%)\end{array}$} & \multirow[t]{2}{*}{$\begin{array}{l}\mathrm{N} \\
(\%)\end{array}$} & \multirow[t]{2}{*}{$\begin{array}{l}\mathrm{C} / \mathrm{N} \\
(\%)\end{array}$} \\
\hline $646 A^{*}$ & $1 \mathrm{H}$ & 1 & 0 & & & & & \\
\hline $646 \mathrm{~A}$ & $1 \mathrm{H}$ & 1 & 45 & 0.45 & 39.00 & 0.89 & 0.08 & 10.60 \\
\hline $646 A^{\star}$ & $1 \mathrm{H}$ & 1 & 92 & 0.92 & 26.00 & & & \\
\hline $646 A^{*}$ & $1 \mathrm{H}$ & 1 & 127 & 1.27 & 7.00 & & & \\
\hline $646 A^{*}$ & $1 \mathrm{H}$ & 2 & 7 & 1.57 & 8.17 & & & \\
\hline $646 A^{\star}$ & $1 \mathrm{H}$ & 2 & 53 & 2.03 & 2.75 & & & \\
\hline $646 A^{*}$ & $1 \mathrm{H}$ & 2 & 110 & 2.60 & 25.25 & & & \\
\hline $646 A^{*}$ & $1 \mathrm{H}$ & 3 & 5 & 3.05 & 6.50 & & & \\
\hline $646 A^{*}$ & $1 \mathrm{H}$ & 3 & 46 & 3.46 & 18.33 & & & \\
\hline $646 \mathrm{~A}$ & $1 \mathrm{H}$ & 3 & 74 & 3.74 & 5.83 & & & \\
\hline $646 A^{*}$ & $1 \mathrm{H}$ & 3 & 106 & 4.06 & 1.58 & & & \\
\hline $646 A^{\star}$ & $1 \mathrm{H}$ & 3 & 128 & 4.28 & 0.00 & & & \\
\hline $646 A^{*}$ & $1 \mathrm{H}$ & 4 & 19 & 4.69 & 7.00 & & & \\
\hline $646 \mathrm{~A}$ & $2 \mathrm{H}$ & 1 & 74 & 5.74 & 6.92 & & & \\
\hline $646 \mathrm{~A}$ & $2 \mathrm{H}$ & 2 & 85 & 7.35 & 6.00 & & & \\
\hline $646 \mathrm{~A}$ & $2 \mathrm{H}$ & 2 & 122 & 7.72 & 7.00 & 0.42 & 0.03 & 15.05 \\
\hline $646 \mathrm{~A}$ & $2 \mathrm{H}$ & 3 & 20 & 8.20 & 11.00 & 0.54 & 0.03 & 19.29 \\
\hline $646 \mathrm{~A}$ & $2 \mathrm{H}$ & 3 & 59 & 8.59 & 9.00 & 0.47 & 0.03 & 16.61 \\
\hline $646 \mathrm{~A}$ & $2 \mathrm{H}$ & 3 & 74 & 8.74 & 10.00 & & & \\
\hline $646 \mathrm{~A}$ & $2 \mathrm{H}$ & 3 & 101 & 9.01 & 7.00 & 0.42 & 0.04 & 11.32 \\
\hline $646 \mathrm{~A}$ & $2 \mathrm{H}$ & 3 & 140 & 9.40 & 9.00 & 0.49 & 0.03 & 15.76 \\
\hline $646 A^{*}$ & $2 \mathrm{H}$ & 4 & 15 & 9.65 & 11.42 & & & \\
\hline $646 \mathrm{~A}$ & $2 \mathrm{H}$ & 4 & 30 & 9.80 & 17.00 & 0.27 & 0.03 & 8.06 \\
\hline $646 \mathrm{~A}$ & $2 \mathrm{H}$ & 4 & 69 & 10.19 & 3.00 & 0.32 & 0.04 & 8.33 \\
\hline $646 A^{\star}$ & $2 \mathrm{H}$ & 4 & 97 & 10.47 & 1.67 & & & \\
\hline $646 \mathrm{~A}$ & $2 \mathrm{H}$ & 4 & 110 & 10.60 & 0.00 & 0.31 & 0.05 & 6.87 \\
\hline $646 A^{*}$ & $2 \mathrm{H}$ & 4 & 134 & 10.84 & 0.58 & & & \\
\hline $646 A^{\star}$ & $2 \mathrm{H}$ & 5 & 15 & 11.15 & 0.83 & & & \\
\hline $646 A^{*}$ & $2 \mathrm{H}$ & 5 & 34 & 11.34 & 5.42 & & & \\
\hline $646 A^{*}$ & $2 \mathrm{H}$ & 5 & 67 & 11.67 & 5.25 & & & \\
\hline $646 A$ & $2 \mathrm{H}$ & 5 & 74 & 11.74 & 6.00 & & & \\
\hline $646 A^{*}$ & $2 \mathrm{H}$ & 5 & 94 & 11.94 & 1.92 & & & \\
\hline $646 A^{*}$ & $2 \mathrm{H}$ & 6 & 4 & 12.54 & 4.25 & & & \\
\hline $646 A^{\star}$ & $2 \mathrm{H}$ & 6 & 57 & 13.07 & 3.58 & & & \\
\hline $646 A^{*}$ & $2 \mathrm{H}$ & 6 & 92 & 13.42 & 5.00 & & & \\
\hline $646 A^{\star}$ & $2 \mathrm{H}$ & 6 & 113 & 13.63 & 1.25 & & & \\
\hline $646 A^{*}$ & $2 \mathrm{H}$ & 6 & 147 & 13.97 & 11.33 & & & \\
\hline $646 A^{\star}$ & $2 \mathrm{H}$ & 7 & 0 & 14.00 & 9.50 & & & \\
\hline $646 \mathrm{~A}$ & $3 \mathrm{H}$ & 1 & 108 & 17.58 & 3.00 & & & \\
\hline $646 \mathrm{~A}$ & $3 \mathrm{H}$ & 2 & 100 & 19.00 & 8.00 & 0.34 & 0.03 & 11.18 \\
\hline $646 \mathrm{~A}$ & $3 \mathrm{H}$ & 2 & 136 & 19.36 & 24.00 & 0.35 & 0.03 & 12.46 \\
\hline $646 \mathrm{~A}$ & $3 \mathrm{H}$ & 3 & 30 & 19.80 & 8.00 & 0.23 & 0.03 & 8.36 \\
\hline $646 \mathrm{~A}$ & $3 \mathrm{H}$ & 3 & 70 & 20.20 & 12.00 & 0.24 & 0.03 & 8.59 \\
\hline $646 \mathrm{~A}$ & $3 \mathrm{H}$ & 3 & 74 & 20.24 & 13.00 & & & \\
\hline $646 \mathrm{~A}$ & $3 \mathrm{H}$ & 3 & 110 & 20.60 & 8.00 & 0.22 & 0.03 & 7.26 \\
\hline $646 A^{*}$ & $3 \mathrm{H}$ & 3 & 137 & 20.87 & 12.42 & & & \\
\hline $646 \mathrm{~A}$ & $3 \mathrm{H}$ & 3 & 149 & 20.99 & 29.00 & 0.37 & 0.03 & 10.82 \\
\hline $646 \mathrm{~A}$ & $3 \mathrm{H}$ & 4 & 3 & 21.03 & 40.00 & & & \\
\hline $646 \mathrm{~A}$ & $3 \mathrm{H}$ & 4 & 18 & 21.18 & 11.00 & & & \\
\hline $646 A^{\star}$ & $3 \mathrm{H}$ & 4 & 34 & 21.34 & 11.17 & & & \\
\hline $646 \mathrm{~A}$ & $3 \mathrm{H}$ & 4 & 36 & 21.36 & 6.00 & 0.34 & 0.04 & 8.06 \\
\hline $646 A^{\star}$ & $3 \mathrm{H}$ & 4 & 53 & 21.53 & 3.17 & & & \\
\hline $646 \mathrm{~A}$ & $3 \mathrm{H}$ & 4 & 82 & 21.82 & 6.00 & 0.29 & 0.03 & 9.09 \\
\hline $646 A^{\star}$ & $3 \mathrm{H}$ & 4 & 105 & 22.05 & 8.58 & & & \\
\hline $646 \mathrm{~A}$ & $3 \mathrm{H}$ & 4 & 116 & 22.16 & 9.00 & 0.37 & 0.04 & 9.51 \\
\hline $646 A^{\star}$ & $3 \mathrm{H}$ & 4 & 146 & 22.46 & 11.58 & & & \\
\hline
\end{tabular}


Appendix A (continued)

\begin{tabular}{|c|c|c|c|c|c|c|c|c|}
\hline \multicolumn{4}{|c|}{$\begin{array}{l}\text { Core, section } \\
\text { depth }(\mathrm{cm})\end{array}$} & \multirow{2}{*}{$\begin{array}{c}\begin{array}{c}\text { Depth } \\
\text { (mbsf) }\end{array} \\
22.56\end{array}$} & \multirow{2}{*}{$\begin{array}{c}\begin{array}{c}\mathrm{CaCO}_{3} \\
(\%)\end{array} \\
15.00\end{array}$} & \multirow{2}{*}{$\begin{array}{c}\begin{array}{c}\text { TOC } \\
(\%)\end{array} \\
0.36\end{array}$} & \multirow{2}{*}{$\begin{array}{c}\begin{array}{c}\mathrm{N} \\
(\%)\end{array} \\
0.04\end{array}$} & \multirow{2}{*}{$\begin{array}{c}\begin{array}{r}\mathrm{C} / \mathrm{N} \\
(\%)\end{array} \\
10.00\end{array}$} \\
\hline $646 \mathrm{~A}$ & $3 \mathrm{H}$ & 5 & 6 & & & & & \\
\hline $646 \mathrm{~A}$ & $3 \mathrm{H}$ & 5 & 46 & 22.96 & 7.00 & 0.27 & 0.03 & 8.60 \\
\hline $646 \mathrm{~A}$ & $3 \mathrm{H}$ & 5 & 48 & 22.98 & 7.25 & & & \\
\hline $646 \mathrm{~A}$ & $3 \mathrm{H}$ & 5 & 83 & 23.33 & 10.00 & 0.20 & 0.03 & 7.62 \\
\hline $646 \mathrm{~A}$ & $4 \mathrm{H}$ & 1 & 140 & 27.60 & 13.83 & & & \\
\hline $646 \mathrm{~A}$ & $4 \mathrm{H}$ & 2 & 6 & 27.76 & 26.00 & 0.22 & 0.03 & 7.99 \\
\hline $646 A^{*}$ & $4 \mathrm{H}$ & 2 & 42 & 28.12 & 20.83 & & & \\
\hline $646 \mathrm{~A}$ & $4 \mathrm{H}$ & 2 & 46 & 28.16 & 33.00 & 0.33 & 0.03 & 11.46 \\
\hline $646 \mathrm{~A}$ & $4 \mathrm{H}$ & 2 & 85 & 28.55 & 27.00 & 0.25 & 0.03 & 8.87 \\
\hline $646 \mathrm{~A}$ & $4 \mathrm{H}$ & 2 & 122 & 28.92 & 34.00 & 0.31 & 0.03 & 10.69 \\
\hline $646 A^{*}$ & $4 \mathrm{H}$ & 2 & 146 & 29.16 & 30.42 & & & \\
\hline $646 \mathrm{~A}$ & $4 \mathrm{H}$ & 3 & 15 & 29.35 & 39.00 & 0.23 & 0.03 & 7.44 \\
\hline $646 \mathrm{~A}$ & $4 \mathrm{H}$ & 3 & 55 & 29.75 & 33.00 & 0.37 & 0.03 & 11.49 \\
\hline $646 A^{\star}$ & $4 \mathrm{H}$ & 3 & 70 & 29.90 & 24.42 & & & \\
\hline $646 A$ & $4 \mathrm{H}$ & 3 & 75 & 29.95 & 22.58 & & & \\
\hline $646 \mathrm{~A}$ & $4 \mathrm{H}$ & 3 & 94 & 30.14 & 17.00 & 0.33 & 0.03 & 11.00 \\
\hline $646 A^{*}$ & $4 \mathrm{H}$ & 3 & 105 & 30.25 & 3.75 & & & \\
\hline $646 \mathrm{~A}$ & $4 \mathrm{H}$ & 3 & 135 & 30.55 & 19.00 & 0.48 & 0.04 & 12.06 \\
\hline $646 A^{*}$ & $4 \mathrm{H}$ & 4 & 0 & 30.70 & 35.17 & & & \\
\hline $646 \mathrm{~A}$ & $4 \mathrm{H}$ & 4 & 25 & 30.95 & 36.00 & 0.31 & 0.03 & 10.37 \\
\hline $646 \mathrm{~A}$ & $4 \mathrm{H}$ & 4 & 65 & 31.35 & 22.00 & 0.35 & 0.03 & 11.04 \\
\hline $646 \mathrm{~A}$ & $4 \mathrm{H}$ & 4 & 105 & 31.75 & 29.00 & 0.23 & 0.02 & 9.31 \\
\hline $646 \mathrm{~A}$ & $4 \mathrm{H}$ & 4 & 146 & 32.16 & 16.00 & 0.31 & 0.03 & 9.75 \\
\hline $646 A^{\star}$ & $4 \mathrm{H}$ & 5 & 1 & 32.21 & 11.33 & & & \\
\hline $646 A^{*}$ & $4 \mathrm{H}$ & 5 & 20 & 32.40 & 13.50 & & & \\
\hline $646 A^{\star}$ & $4 \mathrm{H}$ & 5 & 41 & 32.61 & 16.08 & & & \\
\hline $646 \mathrm{~A}$ & $4 \mathrm{H}$ & 5 & 74 & 32.94 & 17.00 & 0.58 & 0.05 & 11.74 \\
\hline $646 \mathrm{~A}$ & $4 \mathrm{H}$ & 5 & 75 & 32.95 & 14.92 & & & \\
\hline $646 \mathrm{~A}$ & $4 \mathrm{H}$ & 5 & 116 & 33.36 & 5.00 & 0.26 & 0.03 & 8.47 \\
\hline $646 A^{\star}$ & $4 \mathrm{H}$ & 5 & 132 & 33.52 & 0.67 & & & \\
\hline $646 \mathrm{~A}$ & $4 \mathrm{H}$ & 6 & 5 & 33.75 & 0.00 & 0.39 & 0.05 & 8.46 \\
\hline $646 A^{*}$ & $4 \mathrm{H}$ & 6 & 25 & 33.95 & 2.25 & & & \\
\hline $646 \mathrm{~A}$ & $4 \mathrm{H}$ & 6 & 46 & 34.16 & 4.00 & 0.23 & 0.03 & 7.03 \\
\hline $646 A^{\star}$ & $4 \mathrm{H}$ & 6 & 60 & 34.30 & 1.25 & & & \\
\hline $646 \mathrm{~A}$ & $4 \mathrm{H}$ & 6 & 87 & 34.57 & 6.00 & 0.28 & 0.03 & 8.59 \\
\hline $646 A^{\star}$ & $4 \mathrm{H}$ & 6 & 100 & 34.70 & 4.75 & & & \\
\hline $646 A^{*}$ & $4 \mathrm{H}$ & 6 & 113 & 34.83 & 3.92 & & & \\
\hline $646 \mathrm{~A}$ & $4 \mathrm{H}$ & 6 & 125 & 34.95 & 4.00 & 0.23 & 0.03 & 7.40 \\
\hline $646 A^{\star}$ & $5 \mathrm{H}$ & 1 & 0 & 35.80 & 12.58 & & & \\
\hline $646 A^{\star}$ & $5 \mathrm{H}$ & 1 & 20 & 36.00 & 13.92 & & & \\
\hline $646 A^{\star}$ & $5 \mathrm{H}$ & 1 & 60 & 36.40 & 18.25 & & & \\
\hline $646 \mathrm{~A}$ & $5 \mathrm{H}$ & 1 & 88 & 36.68 & 7.00 & 0.34 & 0.05 & 7.56 \\
\hline $646 \mathrm{~A}$ & $5 \mathrm{H}$ & 1 & 99 & 36.79 & 11.17 & & & \\
\hline $646 \mathrm{~A}$ & $5 \mathrm{H}$ & 1 & 125 & 37.05 & 9.00 & 0.41 & 0.05 & 8.12 \\
\hline $646 \mathrm{~A}$ & $5 \mathrm{H}$ & 2 & 1 & 37.31 & 8.00 & 0.34 & 0.04 & 8.34 \\
\hline $646 \mathrm{~A}$ & $5 \mathrm{H}$ & 2 & 17 & 37.47 & 10.00 & 0.41 & 0.04 & 9.51 \\
\hline $646 \mathrm{~A}$ & $5 \mathrm{H}$ & 2 & 44 & 37.74 & 2.00 & 0.37 & 0.04 & 8.40 \\
\hline $646 \mathrm{~A}$ & $5 \mathrm{H}$ & 2 & 55 & 37.85 & 21.00 & 0.25 & 0.03 & 9.09 \\
\hline $646 \mathrm{~A}$ & $5 \mathrm{H}$ & 2 & 82 & 38.12 & 4.00 & 0.36 & 0.04 & 9.03 \\
\hline $646 \mathrm{~A}$ & $5 \mathrm{H}$ & 2 & 94 & 38.24 & 13.00 & 0.33 & 0.04 & 7.52 \\
\hline $646 \mathrm{~A}$ & $5 \mathrm{H}$ & 2 & 120 & 38.50 & 6.00 & 0.27 & 0.03 & 8.21 \\
\hline $646 \mathrm{~A}$ & $5 \mathrm{H}$ & 2 & 136 & 38.66 & 16.00 & 0.37 & 0.05 & 8.28 \\
\hline $646 \mathrm{~A}$ & $5 \mathrm{H}$ & 3 & 8 & 38.88 & 4.00 & 0.35 & 0.05 & 6.70 \\
\hline $646 \mathrm{~A}$ & $5 \mathrm{H}$ & 3 & 25 & 39.05 & 12.00 & 0.50 & 0.06 & 8.56 \\
\hline $646 A$ & $5 \mathrm{H}$ & 3 & 50 & 39.30 & 7.00 & 0.42 & 0.04 & 9.72 \\
\hline $646 \mathrm{~A}$ & $5 \mathrm{H}$ & 3 & 66 & 39.46 & 15.00 & 0.52 & 0.06 & 8.93 \\
\hline $646 \mathrm{~A}$ & $5 \mathrm{H}$ & 3 & 79 & 39.59 & 12.17 & & & \\
\hline
\end{tabular}


Appendix A (continued)

\begin{tabular}{|c|c|c|c|c|c|c|c|c|}
\hline \multicolumn{4}{|c|}{$\begin{array}{l}\text { Core, section } \\
\text { depth }(\mathrm{cm})\end{array}$} & \multirow{2}{*}{$\begin{array}{l}\begin{array}{c}\text { Depth } \\
\text { (mbsf) }\end{array} \\
39.71\end{array}$} & \multirow{2}{*}{$\begin{array}{c}\begin{array}{c}\mathrm{CaCO}_{3} \\
(\%)\end{array} \\
12.00\end{array}$} & \multirow{2}{*}{$\begin{array}{c}\text { TOC } \\
(\%)\end{array}$} & \multirow{2}{*}{$\begin{array}{c}\begin{array}{c}N \\
(\%)\end{array} \\
0.04\end{array}$} & \multirow{2}{*}{$\begin{array}{c}\begin{array}{l}\mathrm{C} / \mathrm{N} \\
(\%)\end{array} \\
8.10\end{array}$} \\
\hline $646 \mathrm{~A}$ & $5 \mathrm{H}$ & 3 & 91 & & & & & \\
\hline $646 \mathrm{~A}$ & $5 \mathrm{H}$ & 3 & 106 & 39.86 & 20.00 & 0.48 & 0.04 & 11.01 \\
\hline $646 \mathrm{~A}$ & $5 \mathrm{H}$ & 3 & 131 & 40.11 & 6.00 & 0.35 & 0.04 & 9.74 \\
\hline $646 \mathrm{~A}$ & $5 \mathrm{H}$ & 3 & 143 & 40.23 & 19.00 & 0.36 & 0.04 & 9.13 \\
\hline $646 \mathrm{~A}$ & $5 \mathrm{H}$ & 4 & 17 & 40.47 & 0.00 & 0.23 & 0.03 & 8.43 \\
\hline $646 \mathrm{~A}$ & $5 \mathrm{H}$ & 4 & 36 & 40.66 & 22.00 & 0.38 & 0.04 & 9.78 \\
\hline $646 \mathrm{~A}$ & $5 \mathrm{H}$ & 4 & 60 & 40.90 & 3.00 & 0.26 & 0.04 & 6.11 \\
\hline $646 \mathrm{~A}$ & $5 \mathrm{H}$ & 4 & 75 & 41.05 & 20.00 & 0.35 & 0.04 & 9.44 \\
\hline $646 \mathrm{~A}$ & $5 \mathrm{H}$ & 4 & 99 & 41.29 & 27.00 & 0.41 & 0.04 & 10.65 \\
\hline $646 \mathrm{~A}$ & $5 \mathrm{H}$ & 4 & 114 & 41.44 & 13.00 & 0.37 & 0.04 & 9.48 \\
\hline $646 \mathrm{~A}$ & $5 \mathrm{H}$ & 4 & 138 & 41.68 & 9.00 & 0.28 & 0.02 & 14.06 \\
\hline $646 \mathrm{~A}$ & $5 \mathrm{H}$ & 4 & 149 & 41.79 & 5.00 & 0.32 & 0.03 & 11.05 \\
\hline $646 \mathrm{~A}$ & $5 \mathrm{H}$ & 5 & 28 & 42.08 & 20.00 & 0.27 & 0.02 & 12.44 \\
\hline $646 A^{\star}$ & $5 \mathrm{H}$ & 5 & 59 & 42.39 & 11.83 & & & \\
\hline $646 \mathrm{~A}$ & $5 \mathrm{H}$ & 5 & 69 & 42.49 & 7.00 & 0.17 & 0.01 & 16.42 \\
\hline $646 \mathrm{~A}$ & $5 \mathrm{H}$ & 5 & 74 & 42.54 & 9.67 & & & \\
\hline $646 A^{*}$ & $5 \mathrm{H}$ & 5 & 89 & 42.69 & 9.50 & & & \\
\hline $646 \mathrm{~A}$ & $5 \mathrm{H}$ & 5 & 108 & 42.88 & 15.00 & 0.56 & 0.04 & 15.69 \\
\hline $646 \mathrm{~A}$ & $5 \mathrm{H}$ & 5 & 149 & 43.29 & 9.00 & 0.35 & 0.03 & 13.92 \\
\hline $646 A^{*}$ & $5 \mathrm{H}$ & 6 & 0 & 43.30 & 20.92 & & & \\
\hline $646 \mathrm{~A}$ & $5 \mathrm{H}$ & 6 & 35 & 43.65 & 14.00 & 0.44 & 0.04 & 12.04 \\
\hline $646 \mathrm{~A}$ & $5 \mathrm{H}$ & 6 & 79 & 44.09 & 11.00 & 0.32 & 0.02 & 13.12 \\
\hline $646 \mathrm{~A}$ & $5 \mathrm{H}$ & 6 & 119 & 44.49 & 10.00 & 0.41 & 0.04 & 11.19 \\
\hline $646 \mathrm{~A}$ & $5 \mathrm{H}$ & 7 & 9 & 44.89 & 14.00 & 0.43 & 0.04 & 10.77 \\
\hline $646 \mathrm{~A}$ & $6 \mathrm{H}$ & 1 & 20 & 45.70 & 1.00 & 0.26 & 0.03 & 8.45 \\
\hline $646 \mathrm{~A}$ & $6 \mathrm{H}$ & 1 & 60 & 46.10 & 8.00 & 0.24 & 0.03 & 8.73 \\
\hline $646 \mathrm{~A}$ & $6 \mathrm{H}$ & 1 & 78 & 46.28 & 7.17 & & & \\
\hline $646 \mathrm{~A}$ & $6 \mathrm{H}$ & 1 & 100 & 46.50 & 3.00 & 0.26 & 0.03 & 9.53 \\
\hline $646 \mathrm{~A}$ & $6 \mathrm{H}$ & 1 & 140 & 46.90 & 3.00 & 0.38 & 0.04 & 9.21 \\
\hline $646 \mathrm{~A}$ & $6 \mathrm{H}$ & 2 & 10 & 47.10 & 37.00 & 0.31 & 0.01 & 21.19 \\
\hline $646 \mathrm{~A}$ & $6 \mathrm{H}$ & 2 & 30 & 47.30 & 0.00 & 0.36 & 0.03 & 10.67 \\
\hline $646 \mathrm{~A}$ & $6 \mathrm{H}$ & 2 & 71 & 47.71 & 1.00 & 0.31 & 0.03 & 9.05 \\
\hline $646 \mathrm{~A}$ & $6 \mathrm{H}$ & 2 & 109 & 48.09 & 0.00 & 0.26 & 0.03 & 9.31 \\
\hline $646 \mathrm{~A}$ & $6 \mathrm{H}$ & 2 & 149 & 48.49 & 0.00 & & & \\
\hline $646 \mathrm{~A}$ & $6 \mathrm{H}$ & 3 & 43 & 48.93 & 0.00 & 0.34 & 0.03 & 10.00 \\
\hline $646 \mathrm{~A}$ & $6 \mathrm{H}$ & 3 & 74 & 49.24 & 4.50 & & & \\
\hline $646 \mathrm{~A}$ & $6 \mathrm{H}$ & 3 & 80 & 49.30 & 2.70 & 0.31 & 0.03 & 8.92 \\
\hline $646 \mathrm{~A}$ & $6 \mathrm{H}$ & 3 & 120 & 49.70 & 0.00 & 0.29 & 0.03 & 8.86 \\
\hline $646 A^{\star}$ & $6 \mathrm{H}$ & 3 & 147 & 49.97 & 1.75 & & & \\
\hline $646 \mathrm{~A}$ & $6 \mathrm{H}$ & 4 & 10 & 50.10 & 0.00 & 0.39 & 0.05 & 8.55 \\
\hline $646 \mathrm{~A}$ & $6 \mathrm{H}$ & 4 & 50 & 50.50 & 0.00 & 0.26 & 0.03 & 8.49 \\
\hline $646 A^{\star}$ & $6 \mathrm{H}$ & 4 & 56 & 50.56 & 3.33 & & & \\
\hline $646 A^{\star}$ & $6 \mathrm{H}$ & 4 & 75 & 50.75 & 12.67 & & & \\
\hline $646 \mathrm{~A}$ & $6 \mathrm{H}$ & 4 & 91 & 50.91 & 10.00 & 0.27 & 0.03 & 8.32 \\
\hline $646 \mathrm{~A}$ & $6 \mathrm{H}$ & 4 & 131 & 51.31 & 8.00 & 0.21 & 0.02 & 8.80 \\
\hline $646 A^{\star}$ & $6 \mathrm{H}$ & 5 & 0 & 51.50 & 10.67 & & & \\
\hline $646 \mathrm{~A}$ & $6 \mathrm{H}$ & 5 & 20 & 51.70 & 10.00 & 0.33 & 0.03 & 10.97 \\
\hline $646 \mathrm{~A}$ & $6 \mathrm{H}$ & 5 & 63 & 52.13 & 1.00 & 0.24 & 0.02 & 12.03 \\
\hline $646 \mathrm{~A}$ & $6 \mathrm{H}$ & 5 & 90 & 52.40 & 12.17 & & & \\
\hline $646 \mathrm{~A}$ & $6 \mathrm{H}$ & 5 & 101 & 52.51 & 9.00 & 0.42 & 0.04 & 10.43 \\
\hline $646 A^{\star}$ & $6 \mathrm{H}$ & 5 & 113 & 52.63 & 12.67 & & & \\
\hline $646 \mathrm{~A}$ & $6 \mathrm{H}$ & 5 & 141 & 52.91 & 8.00 & 0.42 & 0.04 & 10.65 \\
\hline $646 \mathrm{~A}$ & $6 \mathrm{H}$ & 6 & 31 & 53.31 & 7.00 & 0.33 & 0.04 & 9.20 \\
\hline $646 \mathrm{~A}$ & $6 \mathrm{H}$ & 6 & 70 & 53.70 & 6.00 & 0.36 & 0.03 & 11.20 \\
\hline $646 \mathrm{~A}$ & $6 \mathrm{H}$ & 6 & 113 & 54.13 & 9.00 & 0.45 & 0.04 & 11.02 \\
\hline $646 \mathrm{~A}$ & $6 \mathrm{H}$ & 6 & 149 & 54.49 & 6.00 & 0.37 & 0.04 & 9.85 \\
\hline $646 \mathrm{~A}$ & $6 \mathrm{H}$ & 7 & 35 & 54.85 & 0.00 & 0.19 & 0.03 & 7.11 \\
\hline
\end{tabular}


Appendix A (continued)

\begin{tabular}{|c|c|c|c|c|c|c|c|c|}
\hline \multicolumn{4}{|c|}{$\begin{array}{l}\text { Core, section } \\
\text { depth }(\mathrm{cm})\end{array}$} & \multirow{2}{*}{$\begin{array}{c}\begin{array}{c}\text { Depth } \\
\text { (mbsf) }\end{array} \\
55.15\end{array}$} & \multirow{2}{*}{$\begin{array}{c}\begin{array}{c}\mathrm{CaCO}_{3} \\
(\%)\end{array} \\
5.00\end{array}$} & \multirow{2}{*}{$\begin{array}{c}\begin{array}{c}\text { TOC } \\
(\%)\end{array} \\
0.37\end{array}$} & \multirow{2}{*}{$\begin{array}{c}\begin{array}{c}N \\
(\%)\end{array} \\
0.04\end{array}$} & \multirow{2}{*}{$\begin{array}{c}\begin{array}{l}\mathrm{C} / \mathrm{N} \\
(\%)\end{array} \\
9.57\end{array}$} \\
\hline $646 \mathrm{~A}$ & $7 \mathrm{H}$ & 1 & 5 & & & & & \\
\hline $646 A$ & $7 \mathrm{H}$ & 1 & 45 & 55.55 & 27.00 & 0.47 & 0.04 & 11.66 \\
\hline $646 \mathrm{~A}$ & $7 \mathrm{H}$ & 1 & 49 & 55.59 & 15.17 & & & \\
\hline $646 A$ & $7 \mathrm{H}$ & 2 & 95 & 57.55 & 6.00 & 0.25 & 0.03 & 9.42 \\
\hline $646 \mathrm{~A}$ & $7 \mathrm{H}$ & 2 & 135 & 57.95 & 5.00 & 0.23 & 0.03 & 8.72 \\
\hline $646 \mathrm{~A}$ & $7 \mathrm{H}$ & 3 & 13 & 58.23 & 7.00 & 0.21 & 0.03 & 6.93 \\
\hline $646 \mathrm{~A}$ & $7 \mathrm{H}$ & 3 & 54 & 58.64 & 6.00 & 0.20 & 0.03 & 7.60 \\
\hline $646 \mathrm{~A}$ & $7 \mathrm{H}$ & 3 & 74 & 58.84 & 2.83 & & & \\
\hline $646 \mathrm{~A}$ & $7 \mathrm{H}$ & 3 & 74 & 58.84 & 2.83 & & & \\
\hline $646 \mathrm{~A}$ & $7 \mathrm{H}$ & 3 & 94 & 59.04 & 17.00 & 0.26 & 0.04 & 7.22 \\
\hline $646 \mathrm{~A}$ & $7 \mathrm{H}$ & 3 & 94 & 59.04 & 17.00 & 0.26 & 0.04 & 7.22 \\
\hline $646 \mathrm{~A}$ & $7 \mathrm{H}$ & 3 & 138 & 59.48 & 16.00 & 0.23 & 0.02 & 10.53 \\
\hline $646 \mathrm{~A}$ & $7 \mathrm{H}$ & 3 & 138 & 59.48 & 16.00 & 0.23 & 0.02 & 10.53 \\
\hline $646 A^{*}$ & $7 \mathrm{H}$ & 4 & 15 & 59.75 & 15.08 & & & \\
\hline $646 A^{*}$ & $7 \mathrm{H}$ & 4 & 33 & 59.93 & 14.75 & & & \\
\hline $646 \mathrm{~A}$ & $7 \mathrm{H}$ & 4 & 36 & 59.96 & 9.00 & 0.22 & 0.02 & 11.32 \\
\hline $646 \mathrm{~A}$ & $7 \mathrm{H}$ & 4 & 36 & 59.96 & 9.00 & 0.22 & 0.02 & 11.32 \\
\hline $646 A^{*}$ & $7 \mathrm{H}$ & 4 & 54 & 60.14 & 4.17 & & & \\
\hline $646 \mathrm{~A}$ & $7 \mathrm{H}$ & 4 & 75 & 60.35 & 6.00 & 0.31 & 0.03 & 9.19 \\
\hline $646 \mathrm{~A}$ & $7 \mathrm{H}$ & 4 & 75 & 60.35 & 6.00 & 0.31 & 0.03 & 9.19 \\
\hline $646 \mathrm{~A}$ & $7 \mathrm{H}$ & 4 & 115 & 60.75 & 7.00 & 0.20 & 0.02 & 8.64 \\
\hline $646 \mathrm{~A}$ & $7 \mathrm{H}$ & 4 & 115 & 60.75 & 7.00 & 0.20 & 0.02 & 8.64 \\
\hline $646 A^{*}$ & $7 \mathrm{H}$ & 4 & 128 & 60.88 & 6.17 & & & \\
\hline $646 \mathrm{~A}$ & $7 \mathrm{H}$ & 5 & 4 & 61.14 & 7.00 & 0.32 & 0.03 & 10.60 \\
\hline $646 \mathrm{~A}$ & $7 \mathrm{H}$ & 5 & 4 & 61.14 & 7.00 & 0.32 & 0.03 & 10.60 \\
\hline $646 A^{*}$ & $7 \mathrm{H}$ & 5 & 36 & 61.46 & 6.75 & & & \\
\hline $646 \mathrm{~A}$ & $7 \mathrm{H}$ & 5 & 47 & 61.57 & 7.00 & 0.25 & 0.02 & 9.98 \\
\hline $646 \mathrm{~A}$ & $7 \mathrm{H}$ & 5 & 47 & 61.57 & 7.00 & 0.25 & 0.02 & 9.98 \\
\hline $646 \mathrm{~A}$ & $7 \mathrm{H}$ & 5 & 84 & 61.94 & 20.00 & 0.29 & 0.03 & 8.58 \\
\hline $646 \mathrm{~A}$ & $7 \mathrm{H}$ & 5 & 84 & 61.94 & 20.00 & 0.29 & 0.03 & 8.58 \\
\hline $646 A^{*}$ & $7 \mathrm{H}$ & 5 & 87 & 61.97 & 21.00 & & & \\
\hline $646 \mathrm{~A}$ & $7 \mathrm{H}$ & 5 & 125 & 62.35 & 5.00 & 0.36 & 0.03 & 12.89 \\
\hline $646 \mathrm{~A}$ & $7 \mathrm{H}$ & 5 & 125 & 62.35 & 5.00 & 0.36 & 0.03 & 12.89 \\
\hline $646 A^{*}$ & $7 \mathrm{H}$ & 6 & 4 & 62.64 & 5.83 & & & \\
\hline $646 \mathrm{~A}$ & $7 \mathrm{H}$ & 6 & 14 & 62.74 & 9.00 & 0.21 & 0.03 & 6.99 \\
\hline $646 \mathrm{~A}$ & $7 \mathrm{H}$ & 6 & 14 & 62.74 & 9.00 & 0.21 & 0.03 & 6.99 \\
\hline $646 A^{*}$ & $7 \mathrm{H}$ & 6 & 44 & 63.04 & 24.08 & & & \\
\hline $646 \mathrm{~A}$ & $7 \mathrm{H}$ & 6 & 54 & 63.14 & 6.00 & 0.20 & 0.03 & 7.60 \\
\hline $646 \mathrm{~A}$ & $7 \mathrm{H}$ & 6 & 54 & 63.14 & 4.00 & 0.22 & 0.03 & 6.90 \\
\hline $646 \mathrm{~A}$ & $7 \mathrm{H}$ & 6 & 94 & 63.54 & 26.00 & 0.16 & 0.02 & 7.28 \\
\hline $646 \mathrm{~A}$ & $7 \mathrm{H}$ & 6 & 137 & 63.97 & 6.00 & 0.22 & 0.02 & 8.90 \\
\hline $646 \mathrm{~A}$ & $7 \mathrm{H}$ & 7 & 13 & 64.23 & 1.25 & & & \\
\hline $646 \mathrm{~A}$ & $7 \mathrm{H}$ & 7 & 23 & 64.33 & 0.00 & 0.24 & 0.02 & 10.56 \\
\hline $646 \mathrm{~A}$ & $7 \mathrm{H}$ & 7 & 56 & 64.66 & 0.00 & 0.25 & 0.03 & 9.96 \\
\hline $646 \mathrm{~A}$ & $8 \mathrm{H}$ & 1 & 75 & 65.55 & 1.58 & & & \\
\hline $646 \mathrm{~A}$ & $8 \mathrm{H}$ & 3 & 75 & 68.55 & 2.42 & & & \\
\hline $646 \mathrm{~A}$ & $8 \mathrm{H}$ & 5 & 75 & 71.55 & 8.08 & & & \\
\hline $646 \mathrm{~A}$ & $9 \mathrm{H}$ & 1 & 75 & 75.15 & 12.00 & & & \\
\hline $646 \mathrm{~A}$ & $9 \mathrm{H}$ & 3 & 75 & 78.15 & 30.00 & & & \\
\hline $646 \mathrm{~A}$ & $9 \mathrm{H}$ & 5 & 75 & 81.15 & 14.00 & & & \\
\hline $646 \mathrm{~A}$ & $10 \mathrm{H}$ & 1 & 131 & 85.41 & 1.67 & & & \\
\hline $646 \mathrm{~A}$ & $10 \mathrm{H}$ & 3 & 73 & 87.83 & 4.50 & & & \\
\hline $646 \mathrm{~A}$ & $10 \mathrm{H}$ & 5 & 74 & 90.84 & 2.08 & & & \\
\hline $646 \mathrm{~A}$ & $11 \mathrm{H}$ & 1 & 75 & 94.55 & 1.08 & & & \\
\hline $646 \mathrm{~A}$ & $11 \mathrm{H}$ & 3 & 75 & 97.55 & 4.92 & & & \\
\hline $646 \mathrm{~A}$ & $11 \mathrm{H}$ & 5 & 75 & 100.55 & 4.17 & & & \\
\hline $646 \mathrm{~B}$ & $1 \mathrm{H}$ & 1 & 39 & 0.39 & 0.80 & 0.36 & 0.03 & 10.64 \\
\hline
\end{tabular}


Appendix A (continued)

\begin{tabular}{|c|c|c|c|c|c|c|c|c|}
\hline \multicolumn{4}{|c|}{$\begin{array}{l}\text { Core, section } \\
\text { depth }(\mathrm{cm})\end{array}$} & \multirow{2}{*}{$\begin{array}{c}\begin{array}{c}\text { Depth } \\
\text { (mbsf) }\end{array} \\
0.80\end{array}$} & \multirow{2}{*}{$\begin{array}{c}\begin{array}{c}\mathrm{CaCO}_{3} \\
(\%)\end{array} \\
5.60\end{array}$} & \multirow{2}{*}{$\begin{array}{c}\begin{array}{c}\text { TOC } \\
(\%)\end{array} \\
0.31\end{array}$} & \multirow{2}{*}{$\begin{array}{c}\begin{array}{c}N \\
(\%)\end{array} \\
0.02\end{array}$} & \multirow{2}{*}{$\begin{array}{c}\begin{array}{c}\mathrm{C} / \mathrm{N} \\
(\%)\end{array} \\
13.70\end{array}$} \\
\hline $646 B$ & $1 \mathrm{H}$ & 1 & 80 & & & & & \\
\hline $646 \mathrm{~B}$ & $1 \mathrm{H}$ & 1 & 88 & 0.88 & 5.60 & & & \\
\hline $646 \mathrm{~B}$ & $1 \mathrm{H}$ & 1 & 120 & 1.20 & 3.00 & 0.30 & 0.03 & 11.85 \\
\hline $646 \mathrm{~B}$ & $1 \mathrm{H}$ & 2 & 8 & 1.58 & 0.00 & 0.40 & 0.03 & 13.36 \\
\hline $646 \mathrm{~B}$ & $1 \mathrm{H}$ & 2 & 50 & 2.00 & 3.00 & 0.37 & 0.04 & 10.40 \\
\hline $646 \mathrm{~B}$ & $1 \mathrm{H}$ & 2 & 90 & 2.40 & 1.00 & 0.35 & 0.04 & 9.14 \\
\hline $646 \mathrm{~B}$ & $1 \mathrm{H}$ & 2 & 130 & 2.80 & 8.00 & 0.43 & 0.04 & 11.07 \\
\hline $646 \mathrm{~B}$ & $1 \mathrm{H}$ & 3 & 20 & 3.20 & 8.00 & 0.41 & 0.04 & 10.20 \\
\hline $646 B^{*}$ & $1 \mathrm{H}$ & 3 & 51 & 3.51 & 15.00 & & & \\
\hline $646 \mathrm{~B}$ & $1 \mathrm{H}$ & 3 & 60 & 3.60 & 11.00 & 0.56 & 0.04 & 12.65 \\
\hline $646 \mathrm{~B}$ & $1 \mathrm{H}$ & 3 & 70 & 3.70 & 6.00 & & & \\
\hline $646 \mathrm{~B}$ & $1 \mathrm{H}$ & 3 & 100 & 4.00 & 3.00 & 0.36 & 0.03 & 10.43 \\
\hline $646 B$ & $1 \mathrm{H}$ & 3 & 140 & 4.40 & 15.00 & 0.24 & 0.02 & 10.50 \\
\hline $646 \mathrm{~B}$ & $1 \mathrm{H}$ & 4 & 30 & 4.80 & 29.00 & 0.29 & 0.02 & 12.32 \\
\hline $646 B^{*}$ & $1 \mathrm{H}$ & 4 & 60 & 5.10 & 3.08 & & & \\
\hline $646 B^{*}$ & $1 \mathrm{H}$ & 4 & 82 & 5.32 & 7.58 & & & \\
\hline $646 B^{\star}$ & $1 \mathrm{H}$ & 4 & 116 & 5.66 & 8.83 & & & \\
\hline $646 B^{\star}$ & $1 \mathrm{H}$ & 5 & 10 & 6.10 & 10.42 & & & \\
\hline $646 B^{\star}$ & $1 \mathrm{H}$ & 5 & 50 & 6.50 & 5.00 & & & \\
\hline $646 \mathrm{~B}$ & $1 \mathrm{H}$ & 5 & 75 & 6.75 & 6.67 & & & \\
\hline $646 B^{\star}$ & $1 \mathrm{H}$ & 5 & 94 & 6.94 & 1.83 & & & \\
\hline $646 \mathrm{~B}$ & $1 \mathrm{H}$ & 5 & 120 & 7.20 & 0.00 & 0.46 & 0.05 & 9.29 \\
\hline $646 B^{*}$ & $1 \mathrm{H}$ & 5 & 148 & 7.48 & 0.92 & & & \\
\hline $646 B^{\star}$ & $1 \mathrm{H}$ & 6 & 60 & 8.10 & 5.58 & & & \\
\hline $646 \mathrm{~B}$ & $1 \mathrm{H}$ & 6 & 90 & 8.40 & 1.30 & 0.48 & 0.04 & 11.69 \\
\hline $646 \mathrm{~B}$ & $1 \mathrm{H}$ & 6 & 130 & 8.80 & 12.70 & 0.30 & 0.03 & 9.93 \\
\hline $646 B^{*}$ & $1 \mathrm{H}$ & 6 & 137 & 8.87 & 5.83 & & & \\
\hline $646 \mathrm{~B}$ & $2 \mathrm{H}$ & 1 & 60 & 9.60 & 11.80 & 0.38 & 0.04 & 8.90 \\
\hline $646 B$ & $2 \mathrm{H}$ & 1 & 100 & 10.00 & 0.00 & 0.40 & 0.04 & 9.18 \\
\hline $646 \mathrm{~B}$ & $2 \mathrm{H}$ & 1 & 113 & 10.13 & 0.00 & & & \\
\hline $646 \mathrm{~B}$ & $2 \mathrm{H}$ & 1 & 140 & 10.40 & 4.00 & 0.34 & 0.04 & 8.30 \\
\hline $646 B$ & $2 \mathrm{H}$ & 2 & 30 & 10.80 & 0.00 & 0.50 & 0.05 & 10.66 \\
\hline $646 \mathrm{~B}$ & $2 \mathrm{H}$ & 2 & 70 & 11.20 & 7.00 & 0.47 & 0.04 & 10.64 \\
\hline $646 B$ & $2 \mathrm{H}$ & 2 & 110 & 11.60 & 10.00 & 0.34 & 0.04 & 9.37 \\
\hline $646 \mathrm{~B}$ & $2 \mathrm{H}$ & 3 & 0 & 12.00 & 10.00 & 0.25 & 0.03 & 8.90 \\
\hline $646 \mathrm{~B}$ & $2 \mathrm{H}$ & 3 & 39 & 12.39 & 0.00 & 0.39 & 0.05 & 8.40 \\
\hline $646 B^{\star}$ & $2 \mathrm{H}$ & 3 & 50 & 12.50 & 1.33 & & & \\
\hline $646 B^{\star}$ & $2 \mathrm{H}$ & 3 & 70 & 12.70 & 1.83 & & & \\
\hline $646 B$ & $2 \mathrm{H}$ & 3 & 79 & 12.79 & 0.00 & 0.51 & 0.06 & 9.25 \\
\hline $646 \mathrm{~B}$ & $2 \mathrm{H}$ & 3 & 84 & 12.84 & 5.00 & & & \\
\hline $646 B^{\star}$ & $2 \mathrm{H}$ & 3 & 90 & 12.90 & 7.17 & & & \\
\hline $646 \mathrm{~B}$ & $2 \mathrm{H}$ & 3 & 121 & 13.21 & 25.00 & 0.44 & 0.04 & 11.58 \\
\hline $646 B^{\star}$ & $2 \mathrm{H}$ & 3 & 130 & 13.30 & 23.08 & & & \\
\hline $646 \mathrm{~B}$ & $2 \mathrm{H}$ & 4 & 9 & 13.59 & 26.00 & 0.30 & 0.03 & 11.68 \\
\hline $646 B^{\star}$ & $2 \mathrm{H}$ & 4 & 30 & 13.80 & 23.25 & & & \\
\hline $646 B$ & $2 \mathrm{H}$ & 4 & 50 & 14.00 & 12.00 & 0.43 & 0.03 & 15.44 \\
\hline $646 B^{*}$ & $2 \mathrm{H}$ & 4 & 51 & 14.01 & 1.67 & & & \\
\hline $646 \mathrm{~B}$ & $2 \mathrm{H}$ & 4 & 90 & 14.40 & 18.00 & 0.28 & 0.02 & 12.23 \\
\hline $646 B$ & $3 \mathrm{H}$ & 1 & 2 & 14.42 & 5.00 & 0.54 & 0.04 & 12.70 \\
\hline $646 \mathrm{~B}$ & $2 \mathrm{H}$ & 4 & 130 & 14.80 & 6.40 & 0.38 & 0.04 & 10.19 \\
\hline $646 B$ & $3 \mathrm{H}$ & 1 & 42 & 14.82 & 2.00 & & & \\
\hline $646 \mathrm{~B}$ & $2 \mathrm{H}$ & 5 & 20 & 15.20 & 35.00 & 0.27 & 0.03 & 10.58 \\
\hline $646 \mathrm{~B}$ & $3 \mathrm{H}$ & 1 & 82 & 15.22 & 0.00 & 0.39 & 0.04 & 9.05 \\
\hline $646 \mathrm{~B}$ & $3 \mathrm{H}$ & 1 & 93 & 15.33 & 0.00 & & & \\
\hline $646 \mathrm{~B}$ & $2 \mathrm{H}$ & 5 & 60 & 15.60 & 0.00 & 0.30 & 0.03 & 10.88 \\
\hline $646 \mathrm{~B}$ & $3 \mathrm{H}$ & 1 & 122 & 15.62 & 0.00 & 0.44 & 0.05 & 8.69 \\
\hline $646 \mathrm{~B}$ & $2 \mathrm{H}$ & 5 & 67 & 15.67 & 7.00 & & & \\
\hline
\end{tabular}


Appendix A (continued)

\begin{tabular}{|c|c|c|c|c|c|c|c|c|}
\hline \multicolumn{4}{|c|}{$\begin{array}{c}\text { Core, section } \\
\text { depth }(\mathrm{cm})\end{array}$} & \multirow{2}{*}{$\begin{array}{l}\begin{array}{c}\text { Depth } \\
\text { (mbsf) }\end{array} \\
16.00\end{array}$} & \multirow{2}{*}{$\begin{array}{c}\begin{array}{c}\mathrm{CaCO}_{3} \\
(\%)\end{array} \\
3.00\end{array}$} & \multirow{2}{*}{$\begin{array}{c}\begin{array}{c}\text { TOC } \\
(\%)\end{array} \\
0.39\end{array}$} & \multirow{2}{*}{$\begin{array}{c}\begin{array}{c}N \\
(\%)\end{array} \\
0.04\end{array}$} & \multirow{2}{*}{$\begin{array}{c}\begin{array}{c}\mathrm{C} / \mathrm{N} \\
(\%)\end{array} \\
10.24\end{array}$} \\
\hline $646 \mathrm{~B}$ & $2 \mathrm{H}$ & 5 & 100 & & & & & \\
\hline 646B & $3 \mathrm{H}$ & 2 & 12 & 16.02 & 3.00 & 0.53 & 0.05 & 10.51 \\
\hline $646 B^{\star}$ & $3 \mathrm{H}$ & 2 & 20 & 16.10 & 5.42 & & & \\
\hline $646 B$ & $2 \mathrm{H}$ & 5 & 140 & 16.40 & 7.00 & 0.27 & 0.03 & 9.32 \\
\hline $646 \mathrm{~B}$ & $3 \mathrm{H}$ & 2 & 52 & 16.42 & 3.00 & 0.48 & 0.05 & 8.76 \\
\hline $646 B^{\star}$ & $3 \mathrm{H}$ & 2 & 60 & 16.50 & 3.42 & & & \\
\hline $646 \mathrm{~B}$ & $2 \mathrm{H}$ & 6 & 30 & 16.80 & 6.00 & 0.27 & 0.04 & 6.44 \\
\hline $646 B$ & $3 \mathrm{H}$ & 2 & 92 & 16.82 & 0.00 & 0.61 & 0.06 & 10.91 \\
\hline $646 B^{\star}$ & $3 \mathrm{H}$ & 2 & 120 & 17.10 & 4.67 & & & \\
\hline $646 B^{\star}$ & $2 \mathrm{H}$ & 6 & 60 & 17.10 & 3.08 & & & \\
\hline $646 \mathrm{~B}$ & $3 \mathrm{H}$ & 2 & 132 & 17.22 & 1.00 & 0.45 & 0.04 & 11.11 \\
\hline $646 B^{*}$ & $3 \mathrm{H}$ & 3 & 20 & 17.60 & 3.17 & & & \\
\hline $646 \mathrm{~B}$ & $3 \mathrm{H}$ & 3 & 22 & 17.62 & 0.00 & 0.46 & 0.04 & 10.39 \\
\hline $646 \mathrm{~B}$ & $3 \mathrm{H}$ & 3 & 62 & 18.02 & 0.00 & 0.27 & 0.03 & 8.56 \\
\hline $646 \mathrm{~B}$ & $3 \mathrm{H}$ & 3 & 74 & 18.14 & 0.00 & & & \\
\hline $646 \mathrm{~B}$ & $3 \mathrm{H}$ & 3 & 102 & 18.42 & 0.00 & & & \\
\hline $646 \mathrm{~B}$ & $3 \mathrm{H}$ & 3 & 142 & 18.82 & 0.00 & 0.33 & 0.03 & 10.07 \\
\hline $646 \mathrm{~B}$ & $3 \mathrm{H}$ & 4 & 32 & 19.22 & 4.00 & 0.27 & 0.03 & 8.04 \\
\hline $646 B^{\star}$ & $3 \mathrm{H}$ & 4 & 40 & 19.30 & 5.50 & & & \\
\hline $646 B^{\star}$ & $3 \mathrm{H}$ & 4 & 60 & 19.50 & 5.58 & & & \\
\hline $646 B$ & $3 \mathrm{H}$ & 4 & 72 & 19.62 & 9.00 & 0.39 & 0.04 & 10.59 \\
\hline $646 B^{\star}$ & $3 \mathrm{H}$ & 4 & 100 & 19.90 & 21.08 & & & \\
\hline $646 B$ & $3 \mathrm{H}$ & 4 & 112 & 20.02 & 12.00 & 0.32 & 0.03 & 11.06 \\
\hline $646 B^{\star}$ & $3 \mathrm{H}$ & 4 & 122 & 20.12 & 14.33 & & & \\
\hline $646 \mathrm{~B}$ & $3 \mathrm{H}$ & 5 & 2 & 20.42 & 3.00 & 0.35 & 0.03 & 11.15 \\
\hline $646 \mathrm{~B}$ & $3 \mathrm{H}$ & 5 & 42 & 20.82 & 12.00 & 0.41 & 0.04 & 10.48 \\
\hline $646 \mathrm{~B}$ & $3 \mathrm{H}$ & 5 & 58 & 20.98 & 8.00 & & & \\
\hline $646 B$ & $3 \mathrm{H}$ & 5 & 87 & 21.27 & 6.00 & 0.29 & 0.03 & 11.18 \\
\hline $646 B^{*}$ & $3 \mathrm{H}$ & 5 & 100 & 21.40 & 2.58 & & & \\
\hline $646 \mathrm{~B}$ & $3 \mathrm{H}$ & 6 & 7 & 21.97 & 0.00 & 0.39 & 0.05 & 7.26 \\
\hline $646 \mathrm{~B}$ & $3 \mathrm{H}$ & 6 & 47 & 22.37 & 2.00 & & & \\
\hline $646 \mathrm{~B}$ & $3 \mathrm{H}$ & 6 & 87 & 22.77 & 3.00 & 0.27 & 0.03 & 8.59 \\
\hline $646 \mathrm{~B}$ & $3 \mathrm{H}$ & 6 & 127 & 23.17 & 3.00 & 0.25 & 0.03 & 7.97 \\
\hline $646 \mathrm{~B}$ & $3 \mathrm{H}$ & 7 & 17 & 23.57 & 5.00 & 0.26 & 0.03 & 9.26 \\
\hline $646 B^{\star}$ & $3 \mathrm{H}$ & 7 & 40 & 23.80 & 12.42 & & & \\
\hline $646 \mathrm{~B}$ & $4 \mathrm{H}$ & 1 & 2 & 24.12 & 8.00 & 0.47 & 0.03 & 13.85 \\
\hline $646 B^{\star}$ & $4 \mathrm{H}$ & 1 & 33 & 24.43 & 12.00 & & & \\
\hline $646 \mathrm{~B}$ & $4 \mathrm{H}$ & 1 & 40 & 24.50 & 12.00 & 0.34 & 0.03 & 10.38 \\
\hline $646 \mathrm{~B}$ & $4 \mathrm{H}$ & 1 & 50 & 24.60 & 8.30 & & & \\
\hline $646 B^{\star}$ & $4 \mathrm{H}$ & 1 & 73 & 24.83 & 5.50 & & & \\
\hline 646B & $4 \mathrm{H}$ & 1 & 80 & 24.90 & 1.00 & 0.41 & 0.04 & 10.62 \\
\hline $646 B^{\star}$ & $4 \mathrm{H}$ & 1 & 93 & 25.03 & 7.25 & & & \\
\hline 646B & $4 \mathrm{H}$ & 1 & 120 & 25.30 & 0.00 & & & \\
\hline $646 B^{\star}$ & $4 \mathrm{H}$ & 2 & 0 & 25.60 & 0.75 & & & \\
\hline $646 \mathrm{~B}$ & $4 \mathrm{H}$ & 2 & 9 & 25.69 & 0.00 & 0.47 & 0.05 & 10.08 \\
\hline $646 \mathrm{~B}$ & $4 \mathrm{H}$ & 2 & 50 & 26.10 & 10.00 & 0.23 & 0.02 & 10.32 \\
\hline $646 B$ & $4 \mathrm{H}$ & 2 & 91 & 26.51 & 10.00 & 0.29 & 0.03 & 10.19 \\
\hline $646 B^{\star}$ & $4 \mathrm{H}$ & 2 & 110 & 26.70 & 3.00 & & & \\
\hline $646 \mathrm{~B}$ & $4 \mathrm{H}$ & 2 & 130 & 26.90 & 5.00 & 0.34 & 0.03 & 10.39 \\
\hline $646 B$ & $4 \mathrm{H}$ & 3 & 29 & 27.39 & 4.00 & 0.30 & 0.03 & 9.68 \\
\hline $646 \mathrm{~B}$ & $4 \mathrm{H}$ & 3 & 70 & 27.80 & 6.00 & & & \\
\hline $646 \mathrm{~B}$ & $4 \mathrm{H}$ & 3 & 74 & 27.84 & 0.00 & & & \\
\hline $646 \mathrm{~B}$ & $4 \mathrm{H}$ & 3 & 109 & 28.19 & 7.00 & 0.26 & 0.03 & 9.10 \\
\hline $646 B^{*}$ & $4 \mathrm{H}$ & 3 & 126 & 28.36 & 4.25 & & & \\
\hline $646 B$ & $4 \mathrm{H}$ & 4 & 1 & 28.61 & 7.00 & 0.24 & 0.03 & 8.70 \\
\hline 646B & $4 \mathrm{H}$ & 4 & 40 & 29.00 & 19.00 & 0.28 & 0.03 & 10.05 \\
\hline $646 B^{\star}$ & $4 \mathrm{H}$ & 4 & 50 & 29.10 & 19.17 & & & \\
\hline
\end{tabular}


Appendix A (continued)

\begin{tabular}{|c|c|c|c|c|c|c|c|c|}
\hline \multicolumn{4}{|c|}{$\begin{array}{l}\text { Core, section } \\
\text { depth }(\mathrm{cm})\end{array}$} & \multirow{2}{*}{$\begin{array}{c}\begin{array}{c}\text { Depth } \\
\text { (mbsf) }\end{array} \\
29.40\end{array}$} & \multirow{2}{*}{$\begin{array}{c}\begin{array}{c}\mathrm{CaCO}_{3} \\
(\%)\end{array} \\
29.00\end{array}$} & \multirow{2}{*}{$\begin{array}{c}\begin{array}{c}\text { TOC } \\
(\%)\end{array} \\
0.24\end{array}$} & \multirow{2}{*}{$\begin{array}{c}\begin{array}{c}\mathrm{N} \\
(\%)\end{array} \\
0.03\end{array}$} & \multirow{2}{*}{$\begin{array}{c}\begin{array}{c}\mathrm{C} / \mathrm{N} \\
(\%)\end{array} \\
8.65\end{array}$} \\
\hline $646 B$ & $4 \mathrm{H}$ & 4 & 80 & & & & & \\
\hline $646 \mathrm{~B}$ & $4 \mathrm{H}$ & 4 & 120 & 29.80 & 24.00 & 0.39 & 0.03 & 11.69 \\
\hline $646 B^{*}$ & $4 \mathrm{H}$ & 4 & 130 & 29.90 & 18.92 & & & \\
\hline $646 B$ & $4 \mathrm{H}$ & 5 & 10 & 30.20 & 17.00 & 0.27 & 0.03 & 8.01 \\
\hline $646 \mathrm{~B}$ & $4 \mathrm{H}$ & 5 & 25 & 30.35 & 25.42 & & & \\
\hline $646 B$ & $5 \mathrm{H}$ & 1 & 0 & 33.80 & 9.00 & & & \\
\hline $646 B$ & $5 \mathrm{H}$ & 1 & 40 & 34.20 & 12.00 & 0.57 & 0.06 & 10.01 \\
\hline $646 \mathrm{~B}$ & $5 \mathrm{H}$ & 1 & 74 & 34.54 & 11.30 & & & \\
\hline $646 B$ & $5 \mathrm{H}$ & 1 & 80 & 34.60 & 16.00 & 0.50 & 0.05 & 10.82 \\
\hline $646 \mathrm{~B}$ & $5 \mathrm{H}$ & 1 & 120 & 35.00 & 0.00 & 0.59 & 0.03 & 20.62 \\
\hline $646 \mathrm{~B}$ & $5 \mathrm{H}$ & 2 & 10 & 35.40 & 0.00 & 0.41 & 0.03 & 12.89 \\
\hline $646 B$ & $5 \mathrm{H}$ & 2 & 51 & 35.81 & 0.00 & 0.38 & 0.03 & 12.24 \\
\hline $646 \mathrm{~B}$ & $5 \mathrm{H}$ & 2 & 91 & 36.21 & 0.00 & 0.47 & 0.04 & 11.24 \\
\hline $646 B$ & $5 \mathrm{H}$ & 2 & 131 & 36.61 & 0.00 & 0.24 & 0.02 & 11.56 \\
\hline $646 B$ & $5 \mathrm{H}$ & 3 & 29 & 37.09 & 22.00 & 0.43 & 0.03 & 17.16 \\
\hline $646 \mathrm{~B}$ & $5 \mathrm{H}$ & 3 & 70 & 37.50 & 19.00 & 0.55 & 0.03 & 16.72 \\
\hline $646 B$ & $5 \mathrm{H}$ & 3 & 74 & 37.54 & 22.00 & & & \\
\hline $646 \mathrm{~B}$ & $5 \mathrm{H}$ & 3 & 110 & 37.90 & 32.00 & 0.53 & 0.02 & 21.43 \\
\hline $646 \mathrm{~B}$ & $5 \mathrm{H}$ & 3 & 149 & 38.29 & 5.00 & 0.55 & 0.04 & 14.66 \\
\hline $646 B$ & $6 \mathrm{H}$ & 1 & 95 & 44.35 & 14.00 & 0.92 & 0.05 & 19.50 \\
\hline $646 B$ & $6 \mathrm{H}$ & 1 & 99 & 44.39 & 10.20 & & & \\
\hline $646 B$ & $6 \mathrm{H}$ & 1 & 145 & 44.85 & 6.00 & 0.56 & 0.04 & 14.30 \\
\hline $646 B$ & $6 \mathrm{H}$ & 2 & 30 & 45.20 & 10.00 & 0.90 & 0.05 & 19.50 \\
\hline $646 \mathrm{~B}$ & $6 \mathrm{H}$ & 2 & 71 & 45.61 & 9.00 & 0.24 & 0.03 & 7.52 \\
\hline $646 \mathrm{~B}$ & $6 \mathrm{H}$ & 2 & 110 & 46.00 & 11.00 & 0.22 & 0.03 & 7.86 \\
\hline $646 \mathrm{~B}$ & $6 \mathrm{H}$ & 3 & 1 & 46.41 & 13.00 & 0.27 & 0.03 & 10.40 \\
\hline $646 \mathrm{~B}$ & $6 \mathrm{H}$ & 3 & 53 & 46.93 & 30.00 & 0.28 & 0.03 & 10.65 \\
\hline $646 \mathrm{~B}$ & $6 \mathrm{H}$ & 3 & 75 & 47.15 & 14.00 & & & \\
\hline $646 \mathrm{~B}$ & $6 \mathrm{H}$ & 3 & 91 & 47.31 & 14.00 & 0.24 & 0.02 & 11.34 \\
\hline $646 \mathrm{~B}$ & $6 \mathrm{H}$ & 3 & 130 & 47.70 & 13.00 & 0.18 & 0.02 & 8.23 \\
\hline $646 \mathrm{~B}$ & $6 \mathrm{H}$ & 4 & 19 & 48.09 & 10.00 & 0.24 & 0.03 & 9.40 \\
\hline $646 B^{*}$ & $6 \mathrm{H}$ & 4 & 30 & 48.20 & 9.42 & & & \\
\hline $646 \mathrm{~B}$ & $6 \mathrm{H}$ & 4 & 58 & 48.48 & 11.00 & 0.38 & 0.03 & 12.24 \\
\hline $646 \mathrm{~B}$ & $6 \mathrm{H}$ & 4 & 100 & 48.90 & 9.00 & 0.19 & 0.02 & 8.41 \\
\hline $646 B$ & $6 \mathrm{H}$ & 5 & 29 & 49.69 & 7.00 & 0.33 & 0.04 & 8.80 \\
\hline $646 B^{*}$ & $6 \mathrm{H}$ & 5 & 60 & 50.00 & 12.92 & & & \\
\hline $646 \mathrm{~B}$ & $6 \mathrm{H}$ & 5 & 70 & 50.10 & 8.00 & 0.23 & 0.03 & 9.00 \\
\hline $646 \mathrm{~B}$ & $6 \mathrm{H}$ & 5 & 75 & 50.15 & 6.90 & & & \\
\hline $646 \mathrm{~B}$ & $6 \mathrm{H}$ & 5 & 110 & 50.50 & 4.00 & 0.22 & 0.03 & 7.21 \\
\hline $646 \mathrm{~B}$ & $6 \mathrm{H}$ & 5 & 149 & 50.89 & 8.00 & 0.35 & 0.03 & 10.42 \\
\hline $646 \mathrm{~B}$ & $6 \mathrm{H}$ & 6 & 40 & 51.30 & 0.00 & 0.37 & 0.04 & 9.57 \\
\hline $646 \mathrm{~B}$ & $6 \mathrm{H}$ & 6 & 80 & 51.70 & 5.00 & 0.40 & 0.03 & 14.50 \\
\hline $646 \mathrm{~B}$ & $6 \mathrm{H}$ & 6 & 120 & 52.10 & 10.00 & 0.31 & 0.04 & 8.67 \\
\hline $646 B$ & $9 \mathrm{H}$ & 1 & 85 & 73.25 & 13.00 & & & \\
\hline $646 \mathrm{~B}$ & $9 \mathrm{H}$ & 3 & 69 & 76.09 & 0.00 & & & \\
\hline $646 \mathrm{~B}$ & $9 \mathrm{H}$ & 5 & 74 & 79.14 & 0.00 & & & \\
\hline $646 B$ & $10 \mathrm{H}$ & 1 & 95 & 82.95 & 11.00 & & & \\
\hline $646 B$ & $10 \mathrm{H}$ & 3 & 74 & 85.74 & 17.00 & & & \\
\hline $646 \mathrm{~B}$ & $10 \mathrm{H}$ & 5 & 74 & 88.74 & 6.00 & & & \\
\hline $646 \mathrm{~B}$ & $11 \mathrm{H}$ & 1 & 104 & 92.74 & 1.10 & & & \\
\hline $646 \mathrm{~B}$ & $11 \mathrm{H}$ & 3 & 74 & 95.44 & 0.00 & & & \\
\hline $646 \mathrm{~B}$ & $11 \mathrm{H}$ & 5 & 69 & 98.39 & 0.00 & & & \\
\hline $646 \mathrm{~B}$ & $12 \mathrm{H}$ & 1 & 71 & 102.11 & 5.20 & & & \\
\hline $646 \mathrm{~B}$ & $12 \mathrm{H}$ & 3 & 62 & 105.02 & 5.00 & & & \\
\hline $646 \mathrm{~B}$ & $12 \mathrm{H}$ & 5 & 87 & 108.27 & 10.00 & & & \\
\hline $646 \mathrm{~B}$ & $13 \mathrm{H}$ & 1 & 75 & 111.85 & 16.00 & & & \\
\hline $646 \mathrm{~B}$ & $13 \mathrm{H}$ & 3 & 75 & 114.85 & 6.00 & & & \\
\hline
\end{tabular}


Appendix A (continued)

\begin{tabular}{|c|c|c|c|c|c|c|c|c|}
\hline \multicolumn{4}{|c|}{$\begin{array}{l}\text { Core, section } \\
\text { depth }(\mathrm{cm})\end{array}$} & \multirow{2}{*}{$\begin{array}{r}\begin{array}{r}\text { Depth } \\
\text { (mbsf) }\end{array} \\
117.85\end{array}$} & \multirow{2}{*}{$\begin{array}{c}\begin{array}{c}\mathrm{CaCO}_{3} \\
(\%)\end{array} \\
15.00\end{array}$} & \multirow[t]{2}{*}{$\begin{array}{l}\text { TOC } \\
(\%)\end{array}$} & \multirow[t]{2}{*}{$\begin{array}{c}\mathrm{N} \\
(\%)\end{array}$} & \multirow[t]{2}{*}{$\begin{array}{l}\mathrm{C} / \mathrm{N} \\
(\%)\end{array}$} \\
\hline $646 \mathrm{~B}$ & $13 \mathrm{H}$ & 5 & 75 & & & & & \\
\hline $646 \mathrm{~B}$ & $14 \mathrm{H}$ & 1 & 75 & 121.55 & 10.00 & & & \\
\hline $646 \mathrm{~B}$ & $14 \mathrm{H}$ & 3 & 71 & 124.51 & 0.00 & & & \\
\hline $646 B$ & $14 \mathrm{H}$ & 5 & 76 & 127.56 & 7.30 & & & \\
\hline $646 \mathrm{~B}$ & $16 \mathrm{X}$ & 1 & 28 & 140.38 & 10.92 & & & \\
\hline $646 \mathrm{~B}$ & $17 X$ & 1 & 10 & 149.90 & 16.68 & & & \\
\hline $646 \mathrm{~B}$ & $18 \mathrm{X}$ & 1 & 129 & 160.39 & 0.00 & & & \\
\hline $646 B$ & $18 x$ & 3 & 75 & 162.85 & 0.00 & & & \\
\hline $646 \mathrm{~B}$ & $20 x$ & 1 & 74 & 179.24 & 10.00 & & & \\
\hline $646 \mathrm{~B}$ & $20 x$ & 3 & 75 & 182.25 & 5.00 & & & \\
\hline $646 \mathrm{~B}$ & $20 x$ & 5 & 69 & 185.19 & 21.00 & & & \\
\hline $646 \mathrm{~B}$ & $21 x$ & 1 & 98 & 189.18 & 0.00 & & & \\
\hline $646 \mathrm{~B}$ & $21 x$ & 3 & 60 & 191.80 & 0.00 & & & \\
\hline $646 \mathrm{~B}$ & $21 x$ & 4 & 70 & 193.40 & 0.00 & & & \\
\hline $646 \mathrm{~B}$ & $22 \mathrm{X}$ & 1 & 90 & 198.70 & 0.00 & & & \\
\hline $646 B$ & $22 x$ & 6 & 75 & 206.05 & 0.00 & & & \\
\hline $646 \mathrm{~B}$ & $23 x$ & 1 & 73 & 208.23 & 0.00 & & & \\
\hline $646 B$ & $23 x$ & 2 & 69 & 209.69 & 9.00 & & & \\
\hline $646 \mathrm{~B}$ & $23 x$ & 3 & 73 & 211.23 & 0.00 & & & \\
\hline $646 \mathrm{~B}$ & $26 x$ & 1 & 84 & 236.94 & 6.40 & & & \\
\hline $646 \mathrm{~B}$ & $26 x$ & 3 & 83 & 239.93 & 8.00 & & & \\
\hline $646 B$ & $26 x$ & 5 & 75 & 242.85 & 0.00 & & & \\
\hline $646 B$ & $29 X$ & 1 & 71 & 266.01 & 0.00 & & & \\
\hline $646 \mathrm{~B}$ & $29 X$ & 5 & 77 & 272.07 & 9.00 & & & \\
\hline $646 B$ & $30 x$ & 1 & 72 & 275.72 & 12.00 & & & \\
\hline $646 \mathrm{~B}$ & $30 x$ & 3 & 79 & 278.79 & 0.30 & & & \\
\hline $646 \mathrm{~B}$ & $30 x$ & 5 & 81 & 281.81 & 0.00 & & & \\
\hline $646 \mathrm{~B}$ & $31 x$ & 1 & 76 & 285.46 & 7.00 & & & \\
\hline $646 \mathrm{~B}$ & $31 x$ & 3 & 76 & 288.46 & 9.00 & & & \\
\hline $646 B$ & $31 x$ & 5 & 56 & 291.26 & 0.80 & & & \\
\hline $646 B$ & $32 \mathrm{x}$ & 1 & 74 & 295.14 & 0.00 & & & \\
\hline $646 B$ & $32 \mathrm{x}$ & 3 & 60 & 298.00 & 1.00 & & & \\
\hline $646 \mathrm{~B}$ & $33 x$ & 2 & 96 & 306.56 & 4.10 & & & \\
\hline $646 B$ & $33 x$ & 4 & 104 & 309.64 & 0.00 & & & \\
\hline $646 B$ & $35 x$ & 2 & 75 & 325.65 & 3.40 & & & \\
\hline $646 \mathrm{~B}$ & $36 x$ & 3 & 91 & 337.01 & 5.00 & & & \\
\hline $646 \mathrm{~B}$ & $36 x$ & 5 & 77 & 339.87 & 0.00 & & & \\
\hline $646 B$ & $37 x$ & 1 & 69 & 343.49 & 3.00 & & & \\
\hline $646 B$ & $37 x$ & 3 & 85 & 346.65 & 13.00 & & & \\
\hline $646 \mathrm{~B}$ & $37 x$ & 5 & 71 & 349.51 & 3.00 & & & \\
\hline $646 \mathrm{~B}$ & $38 x$ & 1 & 30 & 352.70 & 8.00 & & & \\
\hline $646 B$ & $38 \mathrm{x}$ & 3 & 68 & 356.08 & 3.60 & & & \\
\hline $646 \mathrm{~B}$ & $38 \mathrm{x}$ & 5 & 84 & 359.24 & 12.00 & & & \\
\hline $646 \mathrm{~B}$ & $39 x$ & 1 & 70 & 362.70 & 18.00 & & & \\
\hline $646 B$ & $39 x$ & 3 & 70 & 365.70 & 3.00 & & & \\
\hline $646 B$ & $39 x$ & 5 & 70 & 368.70 & 6.00 & & & \\
\hline $646 \mathrm{~B}$ & $40 x$ & 1 & 75 & 372.45 & 8.17 & & & \\
\hline $646 \mathrm{~B}$ & $40 x$ & 3 & 85 & 375.55 & 4.00 & & & \\
\hline $646 \mathrm{~B}$ & $40 x$ & 6 & 101 & 380.21 & 12.00 & & & \\
\hline $646 B$ & $41 X$ & 2 & 79 & 383.59 & 21.00 & & & \\
\hline $646 B$ & $41 X$ & 4 & 76 & 386.56 & 9.00 & & & \\
\hline $646 B$ & $42 X$ & 2 & 94 & 393.44 & 3.00 & & & \\
\hline $646 B$ & $42 X$ & 5 & 98 & 397.98 & 15.00 & & & \\
\hline $646 B$ & $43 x$ & 3 & 36 & 404.06 & 8.60 & & & \\
\hline $646 B$ & $46 x$ & 1 & 67 & 430.47 & 8.80 & & & \\
\hline $646 B$ & $47 X$ & 1 & 64 & 440.14 & 15.00 & & & \\
\hline $646 \mathrm{~B}$ & $48 x$ & 1 & 100 & 450.10 & 28.00 & & & \\
\hline
\end{tabular}


F. R. HALL ET AL.

Appendix A (continued)

\begin{tabular}{|c|c|c|c|c|c|c|c|c|}
\hline \multicolumn{4}{|c|}{$\begin{array}{l}\text { Core, section } \\
\text { depth }(\mathrm{cm})\end{array}$} & \multirow{2}{*}{$\begin{array}{c}\begin{array}{c}\text { Depth } \\
\text { (mbsf) }\end{array} \\
460.80\end{array}$} & \multirow{2}{*}{$\begin{array}{c}\mathrm{CaCO}_{3} \\
(\%)\end{array}$} & \multirow[t]{2}{*}{$\begin{array}{l}\text { TOC } \\
(\%)\end{array}$} & \multirow[t]{2}{*}{$\begin{array}{l}\mathrm{N} \\
(\%)\end{array}$} & \multirow[t]{2}{*}{$\begin{array}{l}\mathrm{C} / \mathrm{N} \\
(\%)\end{array}$} \\
\hline $646 \mathrm{~B}$ & $49 x$ & 2 & 50 & & & & & \\
\hline $646 \mathrm{~B}$ & $50 x$ & 3 & 33 & 471.83 & 21.00 & & & \\
\hline $646 \mathrm{~B}$ & $51 x$ & 1 & 124 & 479.34 & 1.70 & & & \\
\hline $646 \mathrm{~B}$ & $55 x$ & 2 & 98 & 518.98 & 4.00 & & & \\
\hline $646 \mathrm{~B}$ & $56 x$ & 1 & 139 & 528.09 & 4.58 & & & \\
\hline $646 B$ & $57 X$ & 3 & 76 & 539.56 & 7.00 & & & \\
\hline $646 B$ & $58 x$ & 1 & 146 & 546.96 & 0.00 & & & \\
\hline $646 \mathrm{~B}$ & $60 x$ & 2 & 25 & 566.15 & 0.00 & & & \\
\hline $646 \mathrm{~B}$ & $61 x$ & 1 & 127 & 575.27 & 0.00 & & & \\
\hline $646 \mathrm{~B}$ & $62 x$ & 1 & 54 & 584.14 & 21.67 & & & \\
\hline $646 \mathrm{~B}$ & $65 x$ & 1 & 140 & 613.90 & 7.00 & & & \\
\hline $646 \mathrm{~B}$ & $65 x$ & 4 & 143 & 618.43 & 16.00 & & & \\
\hline $646 B$ & $66 x$ & 3 & 28 & 625.48 & 6.50 & & & \\
\hline $646 \mathrm{~B}$ & $67 x$ & 2 & 38 & 633.68 & 0.00 & & & \\
\hline $646 \mathrm{~B}$ & $68 \mathrm{x}$ & 2 & 107 & 643.87 & 4.60 & & & \\
\hline $646 \mathrm{~B}$ & $69 x$ & 1 & 105 & 651.95 & 22.58 & & & \\
\hline $646 \mathrm{~B}$ & $71 x$ & 1 & 118 & 671.38 & 5.00 & & & \\
\hline $646 \mathrm{~B}$ & $71 x$ & 5 & 95 & 677.15 & 4.00 & & & \\
\hline 646B & $73 x$ & 1 & 94 & 690.24 & 19.00 & & & \\
\hline $646 \mathrm{~B}$ & $73 x$ & 2 & 66 & 691.46 & 33.00 & & & \\
\hline $646 \mathrm{~B}$ & $74 \mathrm{X}$ & 3 & 59 & 702.59 & 4.00 & & & \\
\hline $646 B$ & $75 x$ & 2 & 50 & 710.60 & 0.50 & & & \\
\hline $646 B$ & $75 x$ & 4 & 96 & 714.06 & 0.00 & & & \\
\hline $646 \mathrm{~B}$ & $76 \mathrm{X}$ & 2 & 93 & 720.73 & 1.10 & & & \\
\hline $646 \mathrm{~B}$ & $77 x$ & 4 & 133 & 733.73 & 4.00 & & & \\
\hline $646 \mathrm{~B}$ & $78 x$ & 2 & 1 & 739.21 & 0.60 & & & \\
\hline $646 B$ & $79 x$ & 1 & 128 & 748.68 & 0.00 & & & \\
\hline
\end{tabular}

$\mathrm{TOC}=$ total organic carbon; $\mathrm{C} / \mathrm{N}=$ ratio of carbon to nitrogen.

Samples with * also were measured for magnetic susceptibility. 
Appendix B

Rock-magnetic data collected for Site 646

\begin{tabular}{|c|c|c|c|c|c|c|c|c|c|c|}
\hline \multicolumn{3}{|c|}{$\begin{array}{l}\text { Core, section } \\
\text { depth }(\mathrm{cm})\end{array}$} & \multirow{2}{*}{$\begin{array}{l}\begin{array}{c}\text { Depth } \\
\text { (mbsf) }\end{array} \\
0.00\end{array}$} & \multirow{2}{*}{$\begin{array}{c}\begin{array}{c}\text { Mass } \\
(\mathrm{g})\end{array} \\
6.88\end{array}$} & \multirow{2}{*}{$\begin{array}{c}\begin{array}{c}X \\
\left(10^{-6} \mathrm{~m}^{3} / \mathrm{kg}\right)\end{array} \\
71.94\end{array}$} & \multirow{2}{*}{$\begin{array}{c}\begin{array}{c}X_{\mathrm{ARM}} \\
\left(10^{-6} \mathrm{~m}^{3} / \mathrm{kg}\right)\end{array} \\
7518.33\end{array}$} & \multirow{2}{*}{$\frac{X_{\text {ARM }} / X}{104.50}$} & \multirow{2}{*}{$\begin{array}{c}\begin{array}{c}\text { SIRM } \\
(\mathrm{mA} / \mathrm{m} \cdot \mathrm{kg})\end{array} \\
29.89\end{array}$} & \multirow{2}{*}{$\begin{array}{c}\begin{array}{c}\text { HIRM } \\
(\mathrm{mA} / \mathrm{m} \cdot \mathrm{kg})\end{array} \\
5.99\end{array}$} & \multirow{2}{*}{$\frac{S}{.997}$} \\
\hline $646 A$ & $1 \mathrm{H} 1$ & 0 & & & & & & & & \\
\hline $646 \mathrm{~A}$ & $1 \mathrm{H} 1$ & 110 & 0.10 & 6.14 & 79.18 & 8739.95 & 110.38 & 31.05 & 111.74 & 0.956 \\
\hline $646 \mathrm{~A}$ & $1 \mathrm{H} 1$ & 147 & 0.47 & 6.11 & 72.37 & 7882.50 & 108.91 & 28.26 & 73.66 & 0.968 \\
\hline $646 \mathrm{~A}$ & $1 \mathrm{H} 1$ & 92 & 0.92 & 6.98 & 116.09 & 12886.06 & 111.00 & 43.95 & 73.80 & 0.977 \\
\hline $646 A$ & $1 \mathrm{H} 1$ & 1110 & 1.10 & 6.98 & 145.43 & 14494.75 & 99.67 & 50.24 & 56.24 & 0.984 \\
\hline $646 \mathrm{~A}$ & $1 \mathrm{H} 1$ & 1127 & 1.27 & 7.09 & 184.63 & 14916.44 & 80.79 & 58.86 & 65.70 & 0.984 \\
\hline $646 \mathrm{~A}$ & $1 \mathrm{H} 1$ & 1148 & 1.48 & 6.89 & 180.69 & 15809.82 & 87.50 & 59.71 & 80.62 & 0.981 \\
\hline $646 \mathrm{~A}$ & $1 \mathrm{H} 1$ & 1148 & 1.48 & 7.30 & 176.22 & 15752.05 & 89.39 & 60.13 & 74.04 & 0.982 \\
\hline $646 \mathrm{~A}$ & $1 \mathrm{H} 2$ & 7 & 1.57 & 7.21 & 185.74 & 16968.16 & 91.35 & 64.49 & 94.14 & 0.979 \\
\hline $646 \mathrm{~A}$ & $1 \mathrm{H} 2$ & 53 & 2.03 & 6.40 & 177.25 & 15795.58 & 89.11 & 51.60 & 71.41 & 0.982 \\
\hline $646 \mathrm{~A}$ & 1H 2 & 2110 & 2.60 & 8.88 & 114.88 & 5351.23 & 46.58 & 22.08 & 25.83 & 0.979 \\
\hline $646 \mathrm{~A}$ & $1 \mathrm{H} 2$ & 2130 & 2.80 & 6.87 & 131.11 & 16336.92 & 124.60 & 41.60 & 36.49 & 0.988 \\
\hline $646 \mathrm{~A}$ & $1 \mathrm{H} 2$ & 2142 & 2.92 & 6.64 & 110.49 & 15291.06 & 138.39 & 36.15 & 92.77 & 0.966 \\
\hline $646 \mathrm{~A}$ & $1 \mathrm{H} 2$ & 2142 & 2.92 & 7.08 & 111.97 & 15375.61 & 137.32 & 39.26 & 97.47 & 0.965 \\
\hline $646 \mathrm{~A}$ & $1 \mathrm{H} 3$ & 5 & 3.05 & 7.54 & 102.97 & 12305.35 & 119.51 & 34.05 & 19.12 & 0.992 \\
\hline $646 \mathrm{~A}$ & $1 \mathrm{H} 3$ & 30 & 3.30 & 6.79 & 103.43 & 13053.67 & 126.21 & 33.66 & 78.75 & 0.968 \\
\hline $646 \mathrm{~A}$ & $1 \mathrm{H} 3$ & 46 & 3.46 & 6.85 & 126.18 & 15843.63 & 125.57 & 44.59 & 101.76 & 0.969 \\
\hline $646 \mathrm{~A}$ & $1 \mathrm{H} 3$ & 77 & 3.77 & 6.48 & 147.54 & 18983.42 & 128.67 & 52.49 & 131.15 & 0.968 \\
\hline $646 \mathrm{~A}$ & $1 \mathrm{H} 3$ & 89 & 3.89 & 6.07 & 147.57 & 18717.14 & 126.84 & 48.64 & 130.57 & 0.967 \\
\hline $646 \mathrm{~A}$ & $1 \mathrm{H} 3$ & 3106 & 4.06 & 6.26 & 151.92 & 19689.50 & 129.61 & 53.16 & 157.39 & 0.963 \\
\hline $646 \mathrm{~A}$ & $1 \mathrm{H} 3$ & 3128 & 4.28 & 6.44 & 177.13 & 20362.88 & 114.96 & 60.20 & 167.55 & 0.964 \\
\hline $646 \mathrm{~A}$ & $1 \mathrm{H} 3$ & 3128 & 4.28 & 6.72 & 177.04 & 20802.49 & 117.50 & 64.64 & 152.96 & 0.968 \\
\hline $646 \mathrm{~A}$ & $1 \mathrm{H} 4$ & 5 & 4.55 & 6.74 & 138.12 & 17391.63 & 125.92 & 52.59 & 82.95 & 0.979 \\
\hline $646 \mathrm{~A}$ & $1 \mathrm{H} 4$ & 19 & 4.69 & 6.86 & 123.80 & 15518.31 & 125.35 & 48.49 & 82.12 & 0.977 \\
\hline $646 \mathrm{~A}$ & $1 \mathrm{H} 4$ & 25 & 4.75 & 6.10 & 94.74 & 12223.21 & 129.02 & 34.90 & 158.94 & 0.944 \\
\hline $646 \mathrm{~A}$ & $1 \mathrm{H} 4$ & 25 & 4.75 & 6.41 & 96.43 & 12505.00 & 129.68 & 37.02 & 127.04 & 0.956 \\
\hline $646 \mathrm{~A}$ & $2 \mathrm{H} 1$ & 0 & 5.00 & 7.35 & 179.13 & 14841.88 & 82.86 & 62.80 & 97. & 0.977 \\
\hline $646 \mathrm{~A}$ & $2 \mathrm{H} 1$ & 17 & 5.17 & 5.82 & 162.54 & 14546.31 & 89.49 & 43.49 & 118.17 & 0.968 \\
\hline $646 \mathrm{~A}$ & $2 \mathrm{H} 1$ & 36 & 5.36 & 7.09 & 185.16 & 16513.63 & 89.18 & 60.32 & 100.15 & 0.976 \\
\hline $646 A$ & $2 \mathrm{H} 1$ & 63 & 5.63 & 4.81 & 189.62 & 16780.89 & 88.50 & 41.18 & 117.66 & 0.973 \\
\hline $646 \mathrm{~A}$ & $2 \mathrm{H} 1$ & 77 & 5.77 & 6.82 & 192.31 & 16531.89 & 85.96 & 57.59 & 107.51 & 0.975 \\
\hline $646 A$ & $2 \mathrm{H} 1$ & 92 & 5.92 & 6.92 & 173.92 & 16087.66 & 92.50 & 56.58 & 92.60 & 0.977 \\
\hline $646 \mathrm{~A}$ & $2 \mathrm{H} 1$ & 1115 & 6.15 & 7.24 & 164.32 & 17754.87 & 108.05 & 53.64 & 72.07 & 0.981 \\
\hline $646 \mathrm{~A}$ & $2 \mathrm{H} 1$ & 1115 & 6.15 & 6.81 & 165.66 & 17572.26 & 106.07 & 51.57 & 80.27 & 0.979 \\
\hline $646 \mathrm{~A}$ & $2 \mathrm{H} 2$ & 0 & 6.50 & 6.76 & 124.51 & 14094.91 & 113.20 & 38.33 & 68.49 & 0.976 \\
\hline $646 \mathrm{~A}$ & $2 \mathrm{H} 2$ & 18 & 6.68 & 6.30 & 103.29 & 14261.19 & 138.06 & 30.18 & 52.23 & 0.978 \\
\hline $646 \mathrm{~A}$ & $2 \mathrm{H} 2$ & 35 & 6.85 & 6.73 & 107.15 & 14431.37 & 134.69 & 32.41 & 53.77 & 0.978 \\
\hline $646 \mathrm{~A}$ & $2 \mathrm{H} 2$ & 57 & 7.07 & 6.95 & 136.84 & 17337.23 & 126.70 & 50.39 & 92.22 & 0.975 \\
\hline $646 \mathrm{~A}$ & $2 \mathrm{H} 2$ & 2115 & 7.65 & 6.38 & 107.12 & 17083.06 & 159.48 & 31.49 & 107.03 & 0.957 \\
\hline $646 \mathrm{~A}$ & $2 \mathrm{H} 2$ & 2137 & 7.87 & 7.42 & 152.21 & 17230.02 & 113.20 & 45.57 & 86.01 & 0.972 \\
\hline $646 \mathrm{~A}$ & $2 \mathrm{H} 2$ & 2137 & 7.87 & 7.17 & 162.07 & 19575.21 & 120.78 & 46.89 & 96.84 & 0.970 \\
\hline $646 \mathrm{~A}$ & $2 \mathrm{H} 3$ & 0 & 8.00 & 7.32 & 138.16 & 16093.83 & 116.49 & 43.70 & 71.73 & 0.976 \\
\hline $646 \mathrm{~A}$ & $2 \mathrm{H} 3$ & 28 & 8.28 & 6.89 & 135.11 & 17880.77 & 132.34 & 45.97 & 165.61 & 0.950 \\
\hline $646 \mathrm{~A}$ & $2 \mathrm{H} 3$ & 71 & 8.71 & 7.02 & 129.03 & 17914.89 & 138.84 & 45.45 & 152.33 & 0.953 \\
\hline $646 \mathrm{~A}$ & $2 \mathrm{H} 3$ & 96 & 8.96 & 7.01 & 105.20 & 13489.88 & 128.23 & 33.04 & 72.35 & 0.969 \\
\hline $646 \mathrm{~A}$ & $2 \mathrm{H} 3$ & 3113 & 9.13 & 6.69 & 119.43 & 14186.78 & 118.79 & 34.52 & 92.55 & 0.964 \\
\hline $646 \mathrm{~A}$ & $2 \mathrm{H} 3$ & 3135 & 9.35 & 6.52 & 134.88 & 16951.71 & 125.68 & 44.88 & 124.64 & 0.964 \\
\hline $646 \mathrm{~A}$ & $2 \mathrm{H} 3$ & 3135 & 9.35 & 7.19 & 126.33 & 15364.90 & 121.63 & 44.14 & 101.04 & 0.967 \\
\hline $646 \mathrm{~A}$ & $2 \mathrm{H} 4$ & 0 & 9.50 & 6.91 & 118.17 & 13991.52 & 118.40 & 40.65 & 27.71 & 0.991 \\
\hline $646 \mathrm{~A}$ & $2 \mathrm{H} 4$ & 15 & 9.65 & 6.22 & 102.20 & 11069.82 & 108.32 & 29.16 & 24.73 & 0.989 \\
\hline $646 \mathrm{~A}$ & $2 \mathrm{H} 4$ & 34 & 9.84 & 7.19 & 121.78 & 15242.07 & 125.16 & 45.91 & 51.48 & 0.984 \\
\hline $646 \mathrm{~A}$ & $2 \mathrm{H} 4$ & 57 & 10.07 & 6.60 & 141.62 & 16011.31 & 113.06 & 53.82 & 88.59 & 0.978 \\
\hline $646 A$ & $2 \mathrm{H} 4$ & 97 & 10.47 & 7.22 & 131.20 & 17276.31 & 131.68 & 50.30 & 84.16 & 0.976 \\
\hline $646 \mathrm{~A}$ & $2 \mathrm{H} 4$ & 4115 & 10.65 & 6.36 & 128.99 & 15402.48 & 119.41 & 40.48 & 55.79 & 0.982 \\
\hline $646 \mathrm{~A}$ & $2 \mathrm{H} 4$ & 4134 & 10.84 & 6.81 & 168.80 & 21132.65 & 125.20 & 62.23 & 86.27 & 0.981 \\
\hline $646 \mathrm{~A}$ & $2 \mathrm{H} 4$ & 4134 & 10.84 & 7.45 & 162.39 & 20515.33 & 126.33 & 66.05 & 89.40 & 0.980 \\
\hline
\end{tabular}


Appendix B (continued)

\begin{tabular}{|c|c|c|c|c|c|c|c|c|c|c|c|}
\hline \multicolumn{4}{|c|}{$\begin{array}{l}\text { Core, section } \\
\text { depth }(\mathrm{cm})\end{array}$} & \multirow{2}{*}{$\begin{array}{c}\begin{array}{c}\text { Depth } \\
\text { (mbsf) }\end{array} \\
11.03\end{array}$} & \multirow{2}{*}{$\begin{array}{c}\begin{array}{c}\text { Mass } \\
(\mathrm{g})\end{array} \\
7.03\end{array}$} & \multirow{2}{*}{$\frac{\begin{array}{c}X \\
\left(10^{-6} \mathrm{~m}^{3} / \mathrm{kg}\right)\end{array}}{178.35}$} & \multirow{2}{*}{$\begin{array}{c}\begin{array}{c}X_{\mathrm{ARM}} \\
\left(10^{-6} \mathrm{~m}^{3} / \mathrm{kg}\right)\end{array} \\
21323.72\end{array}$} & \multirow{2}{*}{$\frac{X_{\mathrm{ARM}} / X}{119.56}$} & \multirow{2}{*}{$\begin{array}{c}\begin{array}{c}\text { SIRM } \\
(\mathrm{mA} / \mathrm{m} \cdot \mathrm{kg})\end{array} \\
6 \subseteq .31\end{array}$} & \multirow{2}{*}{$\begin{array}{c}\begin{array}{c}\text { HIRM } \\
(\mathrm{mA} / \mathrm{m} \cdot \mathrm{kg})\end{array} \\
106.77\end{array}$} & \multirow{2}{*}{$\frac{S}{0.978}$} \\
\hline $646 \mathrm{~A}$ & $2 \mathrm{H}$ & 5 & 3 & & & & & & & & \\
\hline $646 \mathrm{~A}$ & $2 \mathrm{H}$ & 5 & 15 & 11.15 & 7.12 & 142.04 & 17900.25 & 126.02 & $5<.62$ & 99.05 & 0.974 \\
\hline $646 \mathrm{~A}$ & $2 \mathrm{H}$ & 5 & 34 & 11.34 & 6.89 & 135.11 & 17166.20 & 127.05 & 5]. .31 & 97.96 & 0.974 \\
\hline $646 \mathrm{~A}$ & $2 \mathrm{H}$ & 5 & 45 & 11.45 & 7.13 & 118.76 & 15595.69 & 131.32 & 48.78 & 97.55 & 0.971 \\
\hline $646 \mathrm{~A}$ & $2 \mathrm{H}$ & 5 & 67 & 11.67 & 6.85 & 125.99 & 15788.98 & 125.31 & 50.04 & 120.16 & 0.967 \\
\hline $646 \mathrm{~A}$ & $2 \mathrm{H}$ & 5 & 94 & 11.94 & 6.19 & 110.61 & 14493.92 & 131.04 & 41.35 & 117.94 & 0.965 \\
\hline $646 \mathrm{~A}$ & $2 \mathrm{H}$ & 5 & 94 & 11.94 & 6.73 & 112.93 & 14598.44 & 129.26 & 45.50 & 117.05 & 0.965 \\
\hline $646 \mathrm{~A}$ & $2 \mathrm{H}$ & 6 & 4 & 12.54 & 5.57 & 148.18 & 16798.53 & 113.36 & 44.65 & 110.49 & 0.972 \\
\hline $646 \mathrm{~A}$ & $2 \mathrm{H}$ & 6 & 34 & 12.84 & 6.69 & 148.73 & 15682.67 & 105.45 & 54.01 & 127.85 & 0.968 \\
\hline $646 \mathrm{~A}$ & $2 \mathrm{H}$ & 6 & 57 & 13.07 & 6.51 & 160.17 & 17108.97 & 106.82 & 57.36 & 115.84 & 0.974 \\
\hline $646 \mathrm{~A}$ & $2 \mathrm{H}$ & 6 & 92 & 13.42 & 5.90 & 131.16 & 15906.23 & 121.27 & 36.74 & 74.62 & 0.976 \\
\hline $646 \mathrm{~A}$ & $2 \mathrm{H}$ & 61 & 113 & 13.63 & 6.18 & 156.12 & 20152.14 & 129.08 & 50.01 & 102.24 & 0.975 \\
\hline $646 \mathrm{~A}$ & $2 \mathrm{H}$ & 61 & 147 & 13.97 & 5.13 & 152.81 & 18650.27 & 122.05 & 37.96 & 88.57 & 0.976 \\
\hline $646 \mathrm{~A}$ & $2 \mathrm{H}$ & 61 & 147 & 13.97 & 5.89 & 140.99 & 16699.16 & 118.45 & 39.74 & 75.69 & 0.978 \\
\hline $646 \mathrm{~A}$ & $2 \mathrm{H}$ & 7 & 0 & 14.00 & 6.46 & 140.02 & 16422.67 & 117.29 & 42.88 & 74.95 & 0.977 \\
\hline $646 \mathrm{~A}$ & $2 \mathrm{H}$ & 7 & 20 & 14.20 & 6.54 & 141.76 & 17924.10 & 126.44 & 47.24 & 91.07 & 0.975 \\
\hline $646 \mathrm{~A}$ & $2 \mathrm{H}$ & 7 & 31 & 14.31 & 6.24 & 137.51 & 17111.80 & 124.44 & 43.41 & 88.31 & 0.975 \\
\hline $646 \mathrm{~A}$ & $2 \mathrm{H}$ & 7 & 31 & 14.31 & 5.85 & 138.08 & 17799.25 & 128.90 & 40.84 & 98.71 & 0.972 \\
\hline $646 \mathrm{~A}$ & $3 \mathrm{H}$ & 1 & 26 & 16.76 & 7.56 & 197.08 & 15903.24 & 80.69 & 72.28 & 22.91 & 0.995 \\
\hline $646 \mathrm{~A}$ & $3 \mathrm{H}$ & 1 & 68 & 17.18 & 7.89 & 181.04 & 18089.53 & 99.92 & 67.21 & 49.35 & 0.988 \\
\hline $646 \mathrm{~A}$ & $3 \mathrm{H}$ & 1 & 84 & 17.34 & 7.16 & 215.99 & 18950.72 & 87.74 & 68.44 & 70.03 & 0.985 \\
\hline $646 \mathrm{~A}$ & $3 \mathrm{H}$ & 11 & 120 & 17.70 & 8.48 & 125.04 & 11905.49 & 95.22 & 44.39 & 57.50 & 0.978 \\
\hline $646 \mathrm{~A}$ & $3 \mathrm{H}$ & 11 & 146 & 17.96 & 6.58 & 114.55 & 14477.78 & 126.38 & 34.03 & 54.60 & 0.979 \\
\hline $646 \mathrm{~A}$ & $3 \mathrm{H}$ & 11 & 146 & 17.96 & 6.66 & 106.20 & 13515.78 & 127.27 & 32.05 & 55.80 & 0.977 \\
\hline $646 \mathrm{~A}$ & $3 \mathrm{H}$ & 2 & 7 & 18.07 & 6.30 & 112.27 & 13843.62 & 123.31 & 31.08 & 66.48 & 0.973 \\
\hline $646 \mathrm{~A}$ & $3 \mathrm{H}$ & 2 & 28 & 18.28 & 6.91 & 107.45 & 15132.65 & 140.84 & 33.92 & 64.13 & 0.974 \\
\hline $646 \mathrm{~A}$ & $3 \mathrm{H}$ & 2 & 66 & 18.66 & 6.55 & 85.93 & 12342.25 & 143.64 & 27.10 & 56.77 & 0.973 \\
\hline $646 A$ & $3 \mathrm{H}$ & 2 & 97 & 18.97 & 6.75 & 156.34 & 19768.10 & 126.45 & 51.99 & 97.00 & 0.975 \\
\hline $646 A$ & $3 \mathrm{H}$ & 21 & 132 & 19.32 & 7.88 & 128.98 & 14553.41 & 112.84 & 44.52 & 80.03 & 0.972 \\
\hline $646 \mathrm{~A}$ & $3 \mathrm{H}$ & 3 & 8 & 19.58 & 6.27 & 157.09 & 19199.87 & 122.23 & 45.05 & 114.04 & 0.968 \\
\hline $646 \mathrm{~A}$ & $3 \mathrm{H}$ & 3 & 46 & 19.96 & 7.22 & 142.68 & 15443.91 & 108.24 & 40.99 & 87.12 & 0.969 \\
\hline $646 \mathrm{~A}$ & $3 \mathrm{H}$ & 3 & 72 & 20.22 & 6.79 & 122.48 & 16380.13 & 133.73 & 42.85 & 61.35 & 0.981 \\
\hline $646 \mathrm{~A}$ & $3 \mathrm{H}$ & 31 & 107 & 20.57 & 6.48 & 145.02 & 18761.44 & 129.38 & 51.67 & 162.04 & 0.959 \\
\hline $646 \mathrm{~A}$ & $3 \mathrm{H}$ & 31 & 137 & 20.87 & 6.76 & 126.56 & 16196.92 & 127.98 & 46.77 & 126.81 & 0.963 \\
\hline $646 A$ & $3 \mathrm{H}$ & 31 & 137 & 20.87 & 6.11 & 127.48 & 16980.77 & 133.20 & 42.22 & 142.03 & 0.959 \\
\hline $646 \mathrm{~A}$ & $3 \mathrm{H}$ & 4 & 34 & 21.34 & 6.73 & 124.88 & 14951.06 & 119.72 & 47.93 & 74.07 & 0.979 \\
\hline $646 A$ & $3 \mathrm{H}$ & 4 & 53 & 21.53 & 6.67 & 175.92 & 20927.16 & 118.96 & 60.69 & 137.52 & 0.970 \\
\hline $646 A$ & $3 \mathrm{H}$ & 4 & 85 & 21.85 & 6.47 & 152.23 & 19515.66 & 128.20 & 48.80 & 42.78 & 0.989 \\
\hline $646 A$ & $3 \mathrm{H}$ & 41 & 105 & 22.05 & 6.32 & 154.65 & 19818.04 & 128.15 & 50.48 & 30.21 & 0.992 \\
\hline $646 \mathrm{~A}$ & $3 \mathrm{H}$ & 41 & 146 & 22.46 & 6.36 & 155.45 & 19036.61 & 122.46 & 54.50 & 47.00 & 0.989 \\
\hline $646 \mathrm{~A}$ & $3 \mathrm{H}$ & 41 & 146 & 22.46 & 7.22 & 161.99 & 19510.64 & 120.44 & 65.42 & 58.20 & 0.987 \\
\hline $646 \mathrm{~A}$ & $3 \mathrm{H}$ & 5 & 0 & 22.50 & 6.37 & 173.95 & 20928.03 & 120.31 & 61.45 & 67.68 & 0.986 \\
\hline $646 \mathrm{~A}$ & $3 \mathrm{H}$ & 5 & 78 & 23.28 & 5.17 & 172.53 & 15291.34 & 88.63 & 46.88 & 74.87 & 0.983 \\
\hline $646 \mathrm{~A}$ & $4 \mathrm{H}$ & 1 & 4 & 26.24 & 6.68 & 146.50 & 17690.06 & 120.75 & & & \\
\hline $646 A$ & $4 \mathrm{H}$ & 1 & 20 & 26.40 & 6.01 & 134.62 & 17612.81 & 130.84 & $40 \quad 59$ & 49.50 & 0.985 \\
\hline $646 \mathrm{~A}$ & $4 \mathrm{H}$ & 1 & 44 & 26.64 & 6.64 & 112.57 & 12742.55 & 113.19 & 3501 & 48.16 & 0.982 \\
\hline $646 A$ & $4 \mathrm{H}$ & 1 & 70 & 26.90 & 7.03 & 110.80 & 12684.58 & 114.49 & 38.95 & 48.58 & 0.982 \\
\hline $646 \mathrm{~A}$ & $4 \mathrm{H}$ & 1 & 90 & 27.10 & 6.97 & 125.81 & 14014.33 & 111.39 & 41.24 & 74.24 & 0.975 \\
\hline $646 \mathrm{~A}$ & $4 \mathrm{H}$ & 11 & 108 & 27.28 & 7.06 & 117.09 & 13059.67 & 111.54 & 38.12 & 45.17 & 0.983 \\
\hline $646 \mathrm{~A}$ & $4 \mathrm{H}$ & 11 & 146 & 27.66 & 6.72 & 143.76 & 17503.07 & 121.75 & 46.14 & 29.02 & 0.992 \\
\hline $646 \mathrm{~A}$ & $4 \mathrm{H}$ & 11 & 146 & 27.66 & 7.07 & 143.75 & 17631.83 & 122.65 & 49.26 & 51.30 & 0.985 \\
\hline $646 A$ & $4 \mathrm{H}$ & 2 & 0 & 27.70 & 6.22 & 121.59 & 16064.65 & 132.12 & 39.46 & 17.32 & 0.995 \\
\hline $646 \mathrm{~A}$ & $4 \mathrm{H}$ & 2 & 20 & 27.90 & 6.04 & 113.36 & 14540.63 & 128.27 & 37.61 & 48.13 & 0.985 \\
\hline $646 \mathrm{~A}$ & $4 \mathrm{H}$ & 2 & 42 & 28.12 & 7.01 & 119.18 & 14494.91 & 121.63 & 45.28 & 47.11 & 0.985 \\
\hline $646 \mathrm{~A}$ & $4 \mathrm{H}$ & 2 & 79 & 28.49 & 6.82 & 137.42 & 15377.29 & 111.90 & $4 \epsilon . .22$ & 49.84 & 0.985 \\
\hline $646 \mathrm{~A}$ & $4 \mathrm{H}$ & 21 & 100 & 28.70 & 6.68 & 103.44 & 13185.50 & 127.47 & 32.36 & 27.29 & 0.989 \\
\hline $646 \mathrm{~A}$ & $4 \mathrm{H}$ & 21 & 146 & 29.16 & 6.55 & 99.35 & 12402.85 & 124.84 & 32.98 & 34.66 & 0.986 \\
\hline
\end{tabular}




\begin{tabular}{|c|c|c|c|c|c|c|c|c|c|c|c|}
\hline \multicolumn{4}{|c|}{$\begin{array}{l}\text { Core, section } \\
\text { depth }(\mathrm{cm})\end{array}$} & \multirow{2}{*}{$\begin{array}{r}\begin{array}{r}\text { Depth } \\
\text { (mbsf) }\end{array} \\
29.16\end{array}$} & \multirow{2}{*}{$\begin{array}{c}\begin{array}{c}\text { Mass } \\
(\mathrm{g})\end{array} \\
7.18\end{array}$} & \multirow{2}{*}{$\begin{array}{c}\begin{array}{c}X \\
\left(10^{-6} \mathrm{~m}^{3} / \mathrm{kg}\right)\end{array} \\
100.26\end{array}$} & \multirow{2}{*}{$\begin{array}{c}\begin{array}{c}X_{\mathrm{ARM}} \\
\left(10^{-6} \mathrm{~m}^{3} / \mathrm{kg}\right)\end{array} \\
12587.13\end{array}$} & \multirow{2}{*}{$\frac{X_{\mathrm{ARM}} / X}{125.55}$} & \multirow{2}{*}{$\begin{array}{c}\begin{array}{c}\text { SIRM } \\
(\mathrm{mA} / \mathrm{m} \cdot \mathrm{kg})\end{array} \\
36.54\end{array}$} & \multirow{2}{*}{$\begin{array}{c}\begin{array}{c}\text { HIRM } \\
(\mathrm{mA} / \mathrm{m} \cdot \mathrm{kg})\end{array} \\
38.92\end{array}$} & \multirow{2}{*}{$\frac{S}{0.985}$} \\
\hline $646 \mathrm{~A}$ & $4 \mathrm{H}$ & 21 & 146 & & & & & & & & \\
\hline $646 \mathrm{~A}$ & $4 \mathrm{H}$ & 3 & 0 & 29.20 & 6.26 & 100.14 & 11766.10 & 117.49 & 31.35 & 66.01 & 0.974 \\
\hline $646 \mathrm{~A}$ & $4 \mathrm{H}$ & 3 & 20 & 29.40 & 6.72 & 111.61 & 11702.67 & 104.86 & 36.31 & 45.27 & 0.983 \\
\hline $646 \mathrm{~A}$ & $4 \mathrm{H}$ & 3 & 51 & 29.71 & 6.84 & 128.75 & 13041.27 & 101.29 & 42.50 & 21.64 & 0.993 \\
\hline $646 \mathrm{~A}$ & $4 \mathrm{H}$ & 3 & 70 & 29.90 & 7.30 & 167.62 & 14930.30 & 89.07 & 57.40 & 82.57 & 0.979 \\
\hline $646 \mathrm{~A}$ & $4 \mathrm{H}$ & 31 & 105 & 30.25 & 6.81 & 150.90 & 20319.84 & 134.66 & 50.19 & 26.16 & 0.993 \\
\hline $646 A$ & $4 \mathrm{H}$ & 31 & 122 & 30.42 & 7.19 & 179.27 & 18904.50 & 105.45 & 63.00 & 102.16 & 0.977 \\
\hline $646 \mathrm{~A}$ & $4 \mathrm{H}$ & 31 & 144 & 30.64 & 6.92 & 139.06 & 14971.53 & 107.66 & 48.82 & 96.70 & 0.973 \\
\hline $646 \mathrm{~A}$ & $4 \mathrm{H}$ & 31 & 144 & 30.64 & 6.53 & 150.45 & 15938.81 & 105.94 & 48.73 & 113.65 & 0.970 \\
\hline $646 \mathrm{~A}$ & $4 \mathrm{H}$ & 4 & 0 & 30.70 & 7.87 & 126.43 & 13108.59 & 103.69 & 50.29 & 94.57 & 0.970 \\
\hline $646 \mathrm{~A}$ & $4 \mathrm{H}$ & 4 & 28 & 30.98 & 6.75 & 119.86 & 12603.76 & 105.16 & 41.89 & 99.67 & 0.968 \\
\hline $646 A$ & $4 \mathrm{H}$ & 4 & 51 & 31.21 & 7.02 & 124.73 & 13463.15 & 107.94 & 44.92 & 101.87 & 0.968 \\
\hline $646 \mathrm{~A}$ & $4 \mathrm{H}$ & 4 & 80 & 31.50 & 6.28 & 101.02 & 13128.54 & 129.96 & 36.06 & 100.13 & 0.965 \\
\hline $646 \mathrm{~A}$ & $4 \mathrm{H}$ & 41 & 119 & 31.89 & 7.11 & 142.94 & 11698.44 & 81.84 & 42.43 & 84.83 & 0.972 \\
\hline $646 A$ & $4 \mathrm{H}$ & 41 & 148 & 32.18 & 7.13 & 162.81 & 15747.57 & 96.73 & 53.46 & 97.52 & 0.974 \\
\hline $646 A$ & $4 \mathrm{H}$ & 41 & 148 & 32.18 & 7.28 & 167.91 & 16352.71 & 97.39 & 56.12 & 111.10 & 0.971 \\
\hline $646 \mathrm{~A}$ & $4 \mathrm{H}$ & 5 & 1 & 32.21 & 7.37 & 158.87 & 15500.16 & 97.57 & 54.11 & 89.66 & 0.976 \\
\hline $646 \mathrm{~A}$ & $4 \mathrm{H}$ & 5 & 19 & 32.39 & 7.25 & 146.94 & 16066.71 & 109.34 & 48.03 & 80.55 & 0.976 \\
\hline $646 \mathrm{~A}$ & $4 \mathrm{H}$ & 5 & 41 & 32.61 & 6.69 & 119.99 & 13877.31 & 115.65 & 34.13 & 53.43 & 0.979 \\
\hline $646 A$ & $4 \mathrm{H}$ & 5 & 56 & 32.76 & 7.53 & 98.10 & 12505.26 & 127.47 & 32.36 & 42.37 & 0.980 \\
\hline $646 \mathrm{~A}$ & $4 \mathrm{H}$ & 5 & 80 & 33.00 & 7.24 & 115.04 & 13679.07 & 118.90 & 35.89 & 51.92 & 0.979 \\
\hline $646 \mathrm{~A}$ & $4 \mathrm{H}$ & 53 & 100 & 33.20 & 6.83 & 112.75 & 15051.83 & 133.49 & 37.64 & 91.97 & 0.967 \\
\hline $646 \mathrm{~A}$ & $4 \mathrm{H}$ & 51 & 132 & 33.52 & 7.06 & 175.81 & 21125.46 & 120.16 & 59.34 & 66.45 & 0.984 \\
\hline $646 \mathrm{~A}$ & $4 \mathrm{H}$ & 53 & 132 & 33.52 & 7.41 & 167.84 & 20102.88 & 119.77 & 57.01 & 108.01 & 0.972 \\
\hline $646 \mathrm{~A}$ & $4 \mathrm{H}$ & 6 & 0 & 33.70 & 6.91 & 156.17 & 20208.68 & 129.40 & 49.15 & 63.12 & 0.982 \\
\hline $646 \mathrm{~A}$ & $4 \mathrm{H}$ & 6 & 25 & 33.95 & 6.28 & 167.84 & 16763.35 & 99.88 & 50.30 & 32.55 & 0.992 \\
\hline $646 A$ & $4 \mathrm{H}$ & 6 & 42 & 34.12 & 7.20 & 148.49 & 20145.17 & 135.67 & 49.89 & 81.13 & 0.977 \\
\hline $646 \mathrm{~A}$ & $4 \mathrm{H}$ & 6 & 60 & 34.30 & 6.48 & 160.14 & 18921.19 & 118.16 & 44.65 & 31.19 & 0.991 \\
\hline $646 \mathrm{~A}$ & $4 \mathrm{H}$ & 6 & 79 & 34.49 & 7.11 & 147.18 & 16840.71 & 114.42 & 43.59 & 36.78 & 0.988 \\
\hline $646 \mathrm{~A}$ & $4 \mathrm{H}$ & 63 & 100 & 34.70 & 6.01 & 127.09 & 15721.49 & 123.70 & 35.71 & 42.35 & 0.986 \\
\hline $646 \mathrm{~A}$ & $4 \mathrm{H}$ & 63 & 113 & 34.83 & 6.65 & 122.61 & 12821.82 & 104.58 & 36.75 & 65.58 & 0.976 \\
\hline $646 A$ & $4 \mathrm{H}$ & 67 & 113 & 34.83 & 6.47 & 121.94 & 12433.50 & 101.96 & 33.93 & 45.74 & 0.983 \\
\hline $646 \mathrm{~A}$ & $5 \mathrm{H}:$ & 1 & 0 & 35.80 & 7.26 & 122.69 & 12101.45 & 98.64 & 43.98 & 14.88 & 0.995 \\
\hline $646 \mathrm{~A}$ & $5 \mathrm{H}$ & 1 & 20 & 36.00 & 7.55 & 120.47 & 13191.14 & 109.50 & 49.32 & 80.52 & 0.975 \\
\hline $646 A$ & $5 \mathrm{H}$ & 1 & 40 & 36.20 & 7.55 & 100.67 & 10585.49 & 105.15 & 37.63 & 48.33 & 0.981 \\
\hline $646 \mathrm{~A}$ & $5 \mathrm{H}:$ & 1 & 60 & 36.40 & 7.20 & 110.10 & 11257.89 & 102.25 & 40.90 & 82.70 & 0.971 \\
\hline $646 A$ & $5 \mathrm{H}$ & 1 & 96 & 36.76 & 7.02 & 158.91 & 17783.90 & 111.91 & 63.78 & 104.46 & 0.977 \\
\hline $646 \mathrm{~A}$ & $5 \mathrm{H}$ & 13 & 148 & 37.28 & 6.24 & 152.61 & 17794.30 & 116.60 & 55.53 & 130.66 & 0.971 \\
\hline $646 \mathrm{~A}$ & $5 \mathrm{H}$ & 11 & 148 & 37.28 & 7.40 & 153.98 & 18254.45 & 118.55 & 69.38 & 116.13 & 0.975 \\
\hline $646 \mathrm{~A}$ & $5 \mathrm{H}$ & 3 & 0 & 37.30 & 6.91 & 115.45 & 14016.29 & 121.41 & 59.70 & 119.33 & 0.972 \\
\hline $646 \mathrm{~A}$ & $5 \mathrm{H}$ & 3 & 20 & 37.50 & 7.43 & 111.93 & 14077.28 & 125.77 & 67.02 & 143.75 & 0.968 \\
\hline $646 A$ & $5 \mathrm{H}$ & 3 & 40 & 37.89 & 6.43 & 120.94 & 15425.69 & 127.55 & 53.17 & 127.17 & 0.969 \\
\hline $646 \mathrm{~A}$ & $5 \mathrm{H}$ & 3 & 60 & 38.02 & 7.70 & 151.08 & 17283.82 & 114.40 & 64.61 & 98.08 & 0.977 \\
\hline $646 \mathrm{~A}$ & $5 \mathrm{H}$ & 3 & 84 & 38.40 & 7.82 & 142.82 & 16384.51 & 114.72 & 47.64 & 81.39 & 0.973 \\
\hline $646 \mathrm{~A}$ & $5 \mathrm{H}$ & 3 & 100 & 38.61 & 7.51 & 147.04 & 17477.53 & 118.86 & 52.84 & 201.39 & 0.943 \\
\hline $646 \mathrm{~A}$ & $5 \mathrm{H}$ & 3 & 130 & 38.61 & 7.56 & 148.89 & 16177.45 & 108.65 & 47.12 & 154.60 & 0.950 \\
\hline $646 \mathrm{~A}$ & $5 \mathrm{H} 3$ & 3 & 130 & 38.80 & 7.44 & 145.72 & 16316.46 & 111.97 & $4 C .53$ & 97.43 & 0.964 \\
\hline $646 \mathrm{~A}$ & $5 \mathrm{H} 2$ & 2 & 0 & 39.00 & 6.55 & 150.18 & 18344.78 & 122.15 & 44.23 & 170.00 & 0.950 \\
\hline $646 \mathrm{~A}$ & $5 \mathrm{H} 2$ & 2 & 20 & 39.20 & 6.60 & 156.27 & 17670.17 & 113.07 & 44.08 & 198.06 & 0.941 \\
\hline $646 \mathrm{~A}$ & $5 \mathrm{H} 2$ & 2 & 59 & 39.40 & 6.85 & 134.25 & 13714.19 & 102.16 & 62.23 & 225.56 & 0.950 \\
\hline $646 \mathrm{~A}$ & $5 \mathrm{H} 2$ & 2 & 72 & 39.64 & 7.73 & 149.84 & 15005.66 & 100.14 & 59.94 & 210.26 & 0.946 \\
\hline $646 \mathrm{~A}$ & $5 \mathrm{H} 2$ & 21 & 110 & 39.80 & 7.89 & 123.08 & 14153.15 & 114.99 & 61.68 & 193.92 & 0.950 \\
\hline $646 A$ & $5 \mathrm{H} 2$ & 21 & 131 & 40.10 & 7.64 & 133.69 & 16307.16 & 121.98 & 63.95 & 85.64 & 0.980 \\
\hline $646 \mathrm{~A}$ & $5 \mathrm{H} 2$ & 21 & 131 & 40.10 & 7.34 & 127.00 & 15844.89 & 124.77 & 63.23 & 234.45 & 0.946 \\
\hline $646 A$ & $5 \mathrm{H}$ & 4 & 0 & 40.30 & 7.65 & 141.39 & 15116.76 & 106.91 & 65.13 & 238.06 & 0.944 \\
\hline $646 \mathrm{~A}$ & $5 \mathrm{H}$ & 4 & 20 & 40.50 & 6.74 & 130.29 & 14034.56 & 107.72 & 51.88 & 224.78 & 0.942 \\
\hline $646 \mathrm{~A}$ & $5 \mathrm{H}$ & 4 & 39 & 40.69 & 7.71 & 115.36 & 12551.41 & 108.80 & 50.58 & 73.19 & 0.978 \\
\hline
\end{tabular}


Appendix B (continued)

\begin{tabular}{|c|c|c|c|c|c|c|c|c|c|c|}
\hline \multicolumn{3}{|c|}{$\begin{array}{l}\text { Core, section } \\
\text { depth }(\mathrm{cm})\end{array}$} & \multirow{2}{*}{$\begin{array}{r}\begin{array}{r}\text { Depth } \\
\text { (mbsf) }\end{array} \\
40.89\end{array}$} & \multirow{2}{*}{$\begin{array}{c}\begin{array}{c}\text { Mass } \\
(\mathrm{g})\end{array} \\
8.04\end{array}$} & \multirow{2}{*}{$\frac{\begin{array}{c}X \\
\left(10^{-6} \mathrm{~m}^{3} / \mathrm{kg}\right)\end{array}}{126.25}$} & \multirow{2}{*}{$\begin{array}{c}\begin{array}{c}X_{\mathrm{ARM}} \\
\left(10^{-6} \mathrm{~m}^{3} / \mathrm{kg}\right)\end{array} \\
13734.41\end{array}$} & \multirow{2}{*}{$\frac{X_{\mathrm{ARM}} / X}{108.78}$} & \multirow{2}{*}{$\begin{array}{c}\begin{array}{c}\text { SIRM } \\
(\mathrm{mA} / \mathrm{m} \cdot \mathrm{kg})\end{array} \\
53.98\end{array}$} & \multirow{2}{*}{$\begin{array}{c}\begin{array}{c}\text { HIRM } \\
(\mathrm{mA} / \mathrm{m} \cdot \mathrm{kg})\end{array} \\
185.80\end{array}$} & \multirow{2}{*}{$\frac{S}{0.945}$} \\
\hline $646 \mathrm{~A}$ & $5 \mathrm{H} 4$ & 59 & & & & & & & & \\
\hline $646 \mathrm{~A}$ & $5 \mathrm{H} 4$ & 80 & 41.10 & 6.87 & 130.20 & 14537.75 & 111.66 & 49.46 & 213.07 & 0.941 \\
\hline $646 \mathrm{~A}$ & $5 \mathrm{H} 4$ & 99 & 41.29 & 6.74 & 139.23 & 15817.40 & 113.60 & 50.17 & 214.63 & 0.942 \\
\hline $646 \mathrm{~A}$ & $5 \mathrm{H} 4$ & 4124 & 41.54 & 7.36 & 145.94 & 16848.17 & 115.45 & 52.24 & 180.23 & 0.949 \\
\hline $646 A$ & $5 \mathrm{H} 4$ & 4145 & 41.75 & 6.38 & 149.45 & 18056.39 & 120.82 & 47.13 & 60.62 & 0.984 \\
\hline $646 A$ & $5 \mathrm{H} 4$ & 4145 & 41.75 & 6.46 & 143.32 & 16958.63 & 118.32 & 45.21 & 174.47 & 0.950 \\
\hline $646 \mathrm{~A}$ & $5 \mathrm{H} 5$ & 0 & 41.80 & 6.96 & 144.94 & 17731.08 & 122.33 & 51.14 & 174.52 & 0.952 \\
\hline $646 \mathrm{~A}$ & $5 \mathrm{H} 5$ & 540 & 42.20 & 6.99 & 169.66 & 21497.51 & 126.71 & 60.72 & 48.04 & 0.989 \\
\hline $646 \mathrm{~A}$ & $5 \mathrm{H} 5$ & $5 \quad 59$ & 42.39 & 7.04 & 171.85 & 20302.70 & 118.14 & 66.45 & 300.39 & 0.936 \\
\hline $646 \mathrm{~A}$ & $5 \mathrm{H} 5$ & 589 & 42.69 & 6.65 & 159.63 & 19595.16 & 122.75 & 54.63 & 205.82 & 0.950 \\
\hline $646 \mathrm{~A}$ & $5 \mathrm{H} 5$ & 89 & 42.69 & 7.05 & 161.45 & 19987.70 & 123.80 & 58.06 & 212.90 & 0.948 \\
\hline $646 \mathrm{~A}$ & $5 \mathrm{H} 6$ & 0 & 43.30 & 6.52 & 135.26 & 16456.52 & 121.66 & 48.21 & 175.66 & 0.952 \\
\hline $646 \mathrm{~A}$ & $5 \mathrm{H}$ & 20 & 43.50 & 6.85 & 160.11 & 18662.29 & 116.56 & 59.38 & 132.88 & 0.969 \\
\hline $646 \mathrm{~A}$ & $5 \mathrm{H} 6$ & 40 & 43.70 & 7.04 & 158.11 & 17063.66 & 107.93 & 52.47 & 85.43 & 0.977 \\
\hline $646 \mathrm{~A}$ & $5 \mathrm{H} 6$ & 60 & 43.90 & 7.30 & 149.38 & 16077.13 & 107.63 & 50.13 & 36.95 & 0.989 \\
\hline $646 \mathrm{~A}$ & $5 \mathrm{H} 6$ & 80 & 44.10 & 7.47 & 98.89 & 12632.94 & 127.75 & 40.48 & 50.03 & 0.982 \\
\hline $646 \mathrm{~A}$ & $5 \mathrm{H}$ & 6126 & 44.56 & 7.38 & 196.78 & 18178.97 & 92.38 & 68.93 & 55.82 & 0.988 \\
\hline $646 \mathrm{~A}$ & $5 \mathrm{H} 6$ & 6126 & 44.56 & 6.87 & 202.25 & 18421.44 & 91.08 & 66.73 & 51.79 & 0.989 \\
\hline $646 \mathrm{~A}$ & $6 \mathrm{H} \mathrm{I}$ & 0 & 45.50 & 7.83 & 123.38 & 13938.32 & 112.97 & 49.43 & 74.21 & 0.976 \\
\hline $646 \mathrm{~A}$ & $6 \mathrm{H}$ & 30 & 45.80 & 6.65 & 115.24 & 13954.55 & 121.09 & 41.18 & 44.99 & 0.985 \\
\hline $646 \mathrm{~A}$ & $6 \mathrm{H} 2$ & 2 & 47.02 & 7.54 & 107.13 & 12557.48 & 117.21 & 34.49 & 39.49 & 0.983 \\
\hline $646 \mathrm{~A}$ & $6 \mathrm{H} 2$ & 55 & 47.55 & 6.86 & 163.72 & 21516.29 & 131.42 & 57.95 & 48.80 & 0.988 \\
\hline $646 \mathrm{~A}$ & $6 \mathrm{H} 2$ & 74 & 47.74 & 7.25 & 139.66 & 18298.22 & 131.02 & 47.42 & 54.30 & 0.983 \\
\hline $646 \mathrm{~A}$ & $6 \mathrm{H} 2$ & 2117 & 48.17 & 7.30 & 172.44 & 23244.30 & 134.80 & 59.27 & 37.42 & 0.991 \\
\hline $646 \mathrm{~A}$ & $6 \mathrm{H} 2$ & 2117 & 48.17 & 7.94 & 173.41 & 23016.06 & 132.73 & 65.87 & 48.11 & 0.988 \\
\hline $646 \mathrm{~A}$ & $6 \mathrm{H} 3$ & 310 & 48.60 & 7.02 & 122.76 & 16917.92 & 137.81 & 41.32 & 43.93 & 0.985 \\
\hline $646 \mathrm{~A}$ & $6 \mathrm{H} 3$ & 34 & 48.84 & 8.18 & 163.26 & 19500.01 & 119.44 & 56.76 & 58.77 & 0.983 \\
\hline $646 \mathrm{~A}$ & $6 \mathrm{H} 3$ & 55 & 49.05 & 6.97 & 166.36 & 22117.04 & 132.94 & 50.86 & 32.46 & 0.991 \\
\hline $646 \mathrm{~A}$ & $6 \mathrm{H} 3$ & 88 & 49.38 & 6.75 & 139.59 & 17738.51 & 127.08 & 43.51 & 44.74 & 0.986 \\
\hline $646 \mathrm{~A}$ & $6 \mathrm{H} 3$ & 3108 & 49.58 & 7.36 & 161.13 & 18649.63 & 115.74 & 50.85 & 37.47 & 0.989 \\
\hline $646 \mathrm{~A}$ & $6 \mathrm{H} 3$ & 3147 & 49.97 & 7.32 & 154.46 & 19105.26 & 123.69 & 55.56 & 66.34 & 0.983 \\
\hline $646 A$ & $6 \mathrm{H} 3$ & 3147 & 49.97 & 7.31 & 149.52 & 18455.82 & 123.44 & & & \\
\hline $646 \mathrm{~A}$ & $6 \mathrm{H} 4$ & 0 & 50.00 & 7.09 & 153.27 & 19818.06 & 129.30 & 51.16 & 12.97 & 0.996 \\
\hline $646 \mathrm{~A}$ & $6 \mathrm{H} 4$ & 56 & 50.56 & 7.67 & 124.32 & 14374.19 & 115.62 & 44.39 & 51.01 & 0.982 \\
\hline $646 \mathrm{~A}$ & $6 \mathrm{H} 4$ & 75 & 50.75 & 7.21 & 146.89 & 18356.87 & 124.97 & 53.40 & 96.93 & 0.974 \\
\hline $646 \mathrm{~A}$ & $6 \mathrm{H} 4$ & 96 & 50.96 & 7.24 & 111.92 & 15051.26 & 134.48 & 43.97 & 74.98 & 0.975 \\
\hline $646 A$ & $6 \mathrm{H} 5$ & 0 & 51.50 & 6.74 & 181.17 & 15341.17 & 84.68 & 56.39 & 94.97 & 0.977 \\
\hline $646 \mathrm{~A}$ & $6 \mathrm{H} 5$ & $5 \quad 17$ & 51.67 & 8.06 & 163.19 & 14960.19 & 91.67 & 6231 & 95.75 & 0.975 \\
\hline $646 \mathrm{~A}$ & $6 \mathrm{H} 5$ & $5 \quad 37$ & 51.87 & 7.76 & 192.33 & 16682.32 & 86.74 & $68 \cdot 58$ & 86.46 & 0.980 \\
\hline $646 \mathrm{~A}$ & $6 \mathrm{H} 5$ & 94 & 52.44 & 7.15 & 167.80 & 17351.27 & 103.41 & 57.86 & 11.09 & 0.997 \\
\hline $646 \mathrm{~A}$ & $6 \mathrm{H} 5$ & 5113 & 52.63 & 7.11 & 165.74 & 18534.66 & 111.83 & 57.27 & 5.60 & 0.999 \\
\hline $646 \mathrm{~A}$ & $6 \mathrm{H} 5$ & 5113 & 52.63 & 7.53 & 167.17 & 18716.26 & 111.96 & 61.14 & 14.83 & 0.996 \\
\hline $646 \mathrm{~A}$ & $6 \mathrm{H} 6$ & 0 & 53.00 & 7.66 & 196.64 & 17268.79 & 87.82 & 62.49 & 70.39 & 0.983 \\
\hline $646 \mathrm{~A}$ & $6 \mathrm{H} 6$ & 17 & 53.17 & 7.34 & 205.22 & 18680.25 & 91.03 & 61.21 & 46.37 & 0.989 \\
\hline $646 \mathrm{~A}$ & $6 \mathrm{H} 6$ & 57 & 53.57 & 7.23 & 164.55 & 17403.23 & 105.76 & 55.30 & 62.82 & 0.984 \\
\hline $646 \mathrm{~A}$ & $6 \mathrm{H} 6$ & 89 & 53.89 & 7.77 & 192.89 & 17860.05 & 92.59 & 61.74 & 40.13 & 0.990 \\
\hline $646 \mathrm{~A}$ & $6 \mathrm{H} 6$ & 6116 & 54.16 & 7.91 & 149.93 & 17239.01 & 114.98 & 54.42 & 10.19 & 0.997 \\
\hline $646 A$ & $6 \mathrm{H} 6$ & 6116 & 54.16 & 6.71 & 148.28 & 17100.03 & 115.32 & 44.41 & 2.87 & 0.999 \\
\hline $646 \mathrm{~A}$ & $6 \mathrm{H} 7$ & 0 & 54.50 & 7.30 & 128.04 & 15518.17 & 121.20 & 41.49 & 5.78 & 0.998 \\
\hline $646 \mathrm{~A}$ & $7 \mathrm{H} 1$ & 0 & 55.10 & 7.93 & 132.44 & 16556.78 & 125.01 & 51.77 & 46.75 & 0.986 \\
\hline $646 \mathrm{~A}$ & $7 \mathrm{H} 1$ & 26 & 55.36 & 7.53 & 103.94 & 13562.00 & 130.48 & 36.95 & 57.62 & 0.977 \\
\hline $646 \mathrm{~A}$ & $7 \mathrm{H} 2$ & 10 & 56.70 & 6.94 & 123.82 & 16571.11 & 133.83 & 40.41 & 70.59 & 0.976 \\
\hline $646 \mathrm{~A}$ & $7 \mathrm{H} 2$ & 2125 & 57.85 & 5.96 & 161.88 & 19050.28 & 117.68 & 47.85 & 88.88 & 0.978 \\
\hline $646 \mathrm{~A}$ & $7 \mathrm{H} 3$ & 5 & 58.15 & 6.89 & 99.74 & 11743.77 & 117.75 & 32.05 & 43.44 & 0.981 \\
\hline $646 \mathrm{~A}$ & $7 \mathrm{H} 3$ & 25 & 58.35 & 6.81 & 162.89 & 17972.76 & 110.34 & 56.53 & 77.61 & 0.981 \\
\hline $646 \mathrm{~A}$ & $7 \mathrm{H} 3$ & 44 & 58.54 & 6.73 & 173.04 & 19177.99 & 110.83 & 62.26 & 107.08 & 0.977 \\
\hline $646 \mathrm{~A}$ & $7 \mathrm{H} 3$ & 387 & 58.97 & 6.89 & 103.93 & 14010.18 & 134.80 & 38.92 & 78.91 & 0.972 \\
\hline
\end{tabular}




\begin{tabular}{|c|c|c|c|c|c|c|c|c|c|c|}
\hline \multicolumn{3}{|c|}{$\begin{array}{l}\text { Core, section } \\
\text { depth }(\mathrm{cm})\end{array}$} & \multirow{2}{*}{$\begin{array}{r}\begin{array}{r}\text { Depth } \\
\text { (mbsf) }\end{array} \\
59.15\end{array}$} & \multirow{2}{*}{$\begin{array}{c}\begin{array}{c}\text { Mass } \\
(\mathrm{g})\end{array} \\
7.16\end{array}$} & \multirow{2}{*}{$\begin{array}{c}\begin{array}{c}X \\
\left(10^{-6} \mathrm{~m}^{3} / \mathrm{kg}\right)\end{array} \\
117.91\end{array}$} & \multirow{2}{*}{$\begin{array}{c}\begin{array}{c}X_{\text {ARM }} \\
\left(10^{-6} \mathrm{~m}^{3} / \mathrm{kg}\right)\end{array} \\
13713.82\end{array}$} & \multirow{2}{*}{$\frac{X_{\mathrm{ARM}} / X}{116.31}$} & \multirow{2}{*}{$\begin{array}{c}\begin{array}{c}\text { SIRM } \\
(\mathrm{mA} / \mathrm{m} \cdot \mathrm{kg})\end{array} \\
41.88\end{array}$} & \multirow{2}{*}{$\begin{array}{c}\begin{array}{c}\text { HIRM } \\
(\mathrm{mA} / \mathrm{m} \cdot \mathrm{kg})\end{array} \\
66.00\end{array}$} & \multirow{2}{*}{$\frac{S}{0.977}$} \\
\hline $646 \mathrm{~A}$ & $7 \mathrm{H} 3$ & 3105 & & & & & & & & \\
\hline $646 A$ & $7 \mathrm{H} 3$ & 3145 & 59.55 & 8.20 & 219.54 & 16269.15 & 74.10 & 72.09 & 45.12 & 0.990 \\
\hline $646 \mathrm{~A}$ & $7 \mathrm{H} 3$ & 3145 & 59.55 & 7.43 & 220.65 & 17242.00 & 78.14 & 64.54 & 40.59 & 0.991 \\
\hline $646 \mathrm{~A}$ & $7 \mathrm{H} 4$ & 15 & 59.75 & 7.32 & 175.23 & 17853.44 & 101.89 & 59.69 & 52.82 & 0.987 \\
\hline $646 \mathrm{~A}$ & $7 \mathrm{H} 4$ & 33 & 59.93 & 8.22 & 147.33 & 14870.73 & 100.93 & 53.65 & 42.41 & 0.987 \\
\hline $646 \mathrm{~A}$ & $7 \mathrm{H} 4$ & 54 & 60.14 & 6.17 & 103.64 & 13439.16 & 129.67 & 26.04 & 42.02 & 0.980 \\
\hline $646 \mathrm{~A}$ & $7 \mathrm{H} 4$ & 78 & 60.38 & 6.87 & 161.65 & 16886.84 & 104.46 & 43.66 & 50.17 & 0.984 \\
\hline $646 \mathrm{~A}$ & $7 \mathrm{H} 4$ & 4128 & 60.88 & 5.03 & 160.09 & 18455.85 & 115.28 & 34.62 & 68.00 & 0.980 \\
\hline $646 \mathrm{~A}$ & $7 \mathrm{H} 4$ & 4128 & 60.88 & 6.62 & 159.79 & 16841.54 & 105.40 & 48.80 & 62.39 & 0.983 \\
\hline $646 \mathrm{~A}$ & $7 \mathrm{H} 5$ & 14 & 61.24 & 5.92 & 168.28 & 21126.11 & 125.54 & 50.31 & 86.91 & 0.980 \\
\hline $646 \mathrm{~A}$ & $7 \mathrm{H} 5$ & 36 & 61.46 & 7.40 & 151.77 & 19368.97 & 127.62 & 63.14 & 112.73 & 0.974 \\
\hline $646 \mathrm{~A}$ & $7 \mathrm{H} 5$ & 54 & 61.64 & 6.76 & 132.32 & 15860.74 & 119.87 & 48.82 & 143.77 & 0.960 \\
\hline $646 \mathrm{~A}$ & $7 \mathrm{H} 5$ & 66 & 61.76 & 6.76 & 136.04 & 15922.25 & 117.05 & 49.62 & 86.35 & 0.976 \\
\hline $646 \mathrm{~A}$ & $7 \mathrm{H} 5$ & $5 \quad 87$ & 61.97 & 5.48 & 124.71 & 15744.78 & 126.25 & 33.37 & 113.93 & 0.963 \\
\hline $646 A$ & $7 \mathrm{H} 5$ & 5106 & 62.16 & 6.35 & 141.46 & 15435.44 & 109.12 & 43.68 & 113.88 & 0.967 \\
\hline $646 \mathrm{~A}$ & $7 \mathrm{H} 5$ & 5106 & 62.16 & 6.82 & 140.00 & 15141.32 & 108.16 & 47.77 & 96.65 & 0.972 \\
\hline $646 A$ & $7 \mathrm{H} 6$ & 4 & 62.64 & 6.91 & 114.54 & 14408.88 & 125.80 & 39.17 & 65.24 & 0.977 \\
\hline $646 \mathrm{~A}$ & $7 \mathrm{H} 6$ & 44 & 63.04 & 7.67 & 137.26 & 15129.59 & 110.23 & 45.19 & 63.82 & 0.978 \\
\hline $646 \mathrm{~A}$ & $7 \mathrm{H} 6$ & 65 & 63.25 & 6.08 & 107.03 & 13931.71 & 130.16 & 33.02 & 77.39 & 0.972 \\
\hline $646 \mathrm{~A}$ & $7 \mathrm{H} 6$ & 86 & 63.46 & 5.73 & 125.41 & 14175.59 & 113.03 & 32.88 & 99.26 & 0.965 \\
\hline $646 \mathrm{~A}$ & $7 \mathrm{H} 6$ & 6113 & 63.73 & 6.92 & 188.99 & 18903.22 & 100.02 & 60.00 & 98.91 & 0.977 \\
\hline $646 \mathrm{~A}$ & $7 \mathrm{H} 6$ & 6142 & 64.02 & 7.35 & 191.95 & 14897.45 & 77.61 & 57.14 & 73.82 & 0.981 \\
\hline $646 \mathrm{~A}$ & $7 \mathrm{H} 6$ & 6142 & 64.02 & 7.58 & 215.46 & 14908.65 & 69.20 & 65.34 & & 0.981 \\
\hline $646 \mathrm{~A}$ & $7 \mathrm{H} 7$ & 0 & 64.82 & 7.41 & 114.78 & 13065.67 & 113.83 & 41.60 & 72.13 & 0.974 \\
\hline $646 \mathrm{~A}$ & $8 \mathrm{H} 1$ & 37 & 65.17 & 7.10 & 94.84 & 13139.11 & 138.54 & 31.97 & 50.65 & 0.978 \\
\hline $646 \mathrm{~A}$ & $8 \mathrm{H} 1$ & 79 & 65.59 & 7.30 & 139.22 & 17350.45 & 124.62 & 52.84 & & \\
\hline $646 \mathrm{~A}$ & $8 \mathrm{H} 1$ & 1102 & 65.82 & 7.23 & 164.20 & 19364.28 & 117.93 & 60.72 & 102.73 & 0.976 \\
\hline $646 \mathrm{~A}$ & $8 \mathrm{H} 1$ & 1115 & 65.95 & 7.20 & 162.97 & 18313.79 & 112.38 & 53.89 & 95.46 & 0.974 \\
\hline $646 \mathrm{~A}$ & $8 \mathrm{H} 1$ & 1115 & 65.95 & 7.52 & 151.86 & 18590.79 & 122.42 & 56.53 & 98.46 & 0.974 \\
\hline $646 \mathrm{~A}$ & $8 \mathrm{H} 2$ & 7 & 66.37 & 7.26 & 103.13 & 12255.15 & 118.83 & 33.16 & 54.84 & 0.976 \\
\hline $646 \mathrm{~A}$ & $8 \mathrm{H} 2$ & 259 & 66.89 & 7.05 & 151.11 & 16756.83 & 110.89 & 49.24 & 73.18 & 0.979 \\
\hline $646 \mathrm{~A}$ & $8 \mathrm{H} 2$ & 2126 & 67.56 & 6.56 & 215.25 & 25161.82 & 116.89 & 78.25 & 167.06 & 0.972 \\
\hline $646 A$ & $8 \mathrm{H} 2$ & 2145 & 67.75 & 7.06 & 231.50 & 25197.34 & 108.84 & 85.24 & 165.86 & 0.973 \\
\hline $646 \mathrm{~A}$ & $8 \mathrm{H} 2$ & 2145 & 67.75 & 7.35 & 231.60 & 25182.03 & 108.73 & 88.73 & 134.28 & 0.978 \\
\hline $646 \mathrm{~A}$ & $8 \mathrm{H} 3$ & 5 & 67.85 & 6.89 & 175.04 & 16311.42 & 93.19 & 48.15 & 89.37 & 0.974 \\
\hline $646 \mathrm{~A}$ & $8 \mathrm{H} 3$ & 20 & 68.00 & 6.68 & 159.86 & 18816.20 & 117.71 & 55.37 & 116.85 & 0.972 \\
\hline $646 \mathrm{~A}$ & $8 \mathrm{H} 3$ & 57 & 68.37 & 6.96 & 121.12 & 14905.48 & 123.07 & 45.34 & 78.68 & 0.976 \\
\hline $646 \mathrm{~A}$ & $8 \mathrm{H} 3$ & 3101 & 68.81 & 7.17 & 131.94 & 15855.78 & 120.18 & 52.95 & 145.30 & 0.961 \\
\hline $646 A$ & $8 \mathrm{H}$ & 3146 & 69.26 & 6.90 & 178.25 & 15093.40 & 84.68 & 57.10 & 149.64 & 0.964 \\
\hline $646 \mathrm{~A}$ & $8 \mathrm{H} 3$ & 3146 & 69.26 & 7.37 & 178.98 & 15045.04 & 84.06 & 61.74 & 134.30 & 0.968 \\
\hline $646 \mathrm{~A}$ & $8 \mathrm{H}$ & 6 & 69.36 & 7.57 & 177.57 & 14428.32 & 81.25 & 57.08 & 119.20 & 0.968 \\
\hline $646 \mathrm{~A}$ & $8 \mathrm{H}$ & 46 & 69.76 & 8.12 & 237.02 & 17129.68 & 72.27 & 79.00 & 143.92 & 0.970 \\
\hline $646 A$ & $8 \mathrm{H}$ & 76 & 70.06 & 7.54 & 138.46 & 16209.86 & 117.07 & 53.03 & 115.70 & 0.967 \\
\hline $646 \mathrm{~A}$ & $8 \mathrm{H}$ & 90 & 70.20 & 6.65 & 157.55 & 16348.28 & 103.76 & 49.53 & 112.49 & 0.970 \\
\hline $646 \mathrm{~A}$ & $8 \mathrm{H}$ & 4107 & 70.37 & 5.32 & 172.62 & 16263.18 & 94.21 & 37.37 & 52.86 & 0.985 \\
\hline $646 \mathrm{~A}$ & $8 \mathrm{H}$ & 4107 & 70.37 & 4.82 & 163.94 & 16521.24 & 100.77 & 35.77 & 72.13 & 0.981 \\
\hline $646 \mathrm{~A}$ & $8 \mathrm{H} 5$ & 0 & 70.80 & 6.63 & 162.39 & 21621.68 & 133.15 & 59.79 & 101.71 & 0.977 \\
\hline $646 \mathrm{~A}$ & $8 \mathrm{H} 5$ & 34 & 71.14 & 7.74 & 117.67 & 13728.53 & 116.66 & 46.28 & 73.14 & 0.976 \\
\hline $646 \mathrm{~A}$ & $8 \mathrm{H} 5$ & 590 & 71.70 & 7.69 & 111.09 & 11360.12 & 102.26 & 54.15 & 72.39 & 0.979 \\
\hline $646 \mathrm{~A}$ & $8 \mathrm{H}$ & 5113 & 71.93 & 6.59 & 154.60 & 20075.34 & 129.85 & 56.49 & 122.79 & 0.971 \\
\hline $646 \mathrm{~A}$ & $8 \mathrm{H}$ & 5145 & 72.25 & 6.07 & 184.61 & 22735.21 & 123.15 & 58.50 & 138.31 & 0.971 \\
\hline $646 \mathrm{~A}$ & $8 \mathrm{H}$ & 5145 & 72.25 & 6.11 & 187.11 & 22833.27 & 122.03 & 58.79 & 97.08 & 0.980 \\
\hline $646 \mathrm{~A}$ & $9 \mathrm{H}$ & 111 & 74.51 & 7.25 & 125.11 & 15560.39 & 124.38 & 55.50 & 154.06 & 0.960 \\
\hline $646 \mathrm{~A}$ & $9 \mathrm{H}$ & 142 & 74.82 & 7.68 & 125.30 & 13569.80 & 108.30 & 52.10 & 90.47 & 0.973 \\
\hline $646 \mathrm{~A}$ & $9 \mathrm{H}$ & 69 & 75.09 & 8.59 & 126.21 & 12272.07 & 97.23 & 56.13 & 135.50 & 0.959 \\
\hline $646 \mathrm{~A}$ & $9 \mathrm{H}$ & 93 & 75.33 & 7.84 & 142.93 & 16742.83 & 117.14 & 55.25 & 115.65 & 0.967 \\
\hline $646 \mathrm{~A}$ & $9 \mathrm{H}$ & 1116 & 75.56 & 7.57 & 168.94 & 21408.40 & 126.72 & 68.14 & 93.02 & 0.979 \\
\hline
\end{tabular}


Appendix B (continued)

\begin{tabular}{|c|c|c|c|c|c|c|c|c|c|c|c|}
\hline \multicolumn{4}{|c|}{$\begin{array}{l}\text { Core, section } \\
\text { depth }(\mathrm{cm})\end{array}$} & \multirow{2}{*}{$\begin{array}{c}\begin{array}{c}\text { Depth } \\
\text { (mbsf) }\end{array} \\
75.86\end{array}$} & \multirow{2}{*}{$\begin{array}{c}\begin{array}{c}\text { Mass } \\
(\mathrm{g})\end{array} \\
7.69\end{array}$} & \multirow{2}{*}{$\frac{\begin{array}{c}X \\
\left(10^{-6} \mathrm{~m}^{3} / \mathrm{kg}\right)\end{array}}{178.56}$} & \multirow{2}{*}{$\begin{array}{c}\begin{array}{c}X_{\mathrm{ARM}} \\
\left(10^{-6} \mathrm{~m}^{3} / \mathrm{kg}\right)\end{array} \\
24288.87\end{array}$} & \multirow{2}{*}{$\frac{X_{\mathrm{ARM}} / X}{136.03}$} & \multirow{2}{*}{$\begin{array}{c}\begin{array}{c}\text { SIRM } \\
(\mathrm{mA} / \mathrm{m} \cdot \mathrm{kg})\end{array} \\
77.70\end{array}$} & \multirow{2}{*}{$\begin{array}{c}\begin{array}{c}\text { HIRM } \\
(\mathrm{mA} / \mathrm{m} \cdot \mathrm{kg})\end{array} \\
91.16\end{array}$} & \multirow{2}{*}{$\frac{S}{0.982}$} \\
\hline $646 \mathrm{~A}$ & $9 \mathrm{H}$ & 1 & 146 & & & & & & & & \\
\hline $646 \mathrm{~A}$ & $9 \mathrm{H}$ & 1 & 146 & 75.86 & 6.60 & 184.44 & 24755.79 & 134.22 & 65.77 & 89.45 & 0.982 \\
\hline $646 A$ & $9 \mathrm{H}$ & 2 & 0 & 75.90 & 7.10 & 176.41 & 23829.89 & 135.08 & 69.62 & 99.83 & 0.980 \\
\hline $646 \mathrm{~A}$ & $9 \mathrm{H}$ & 2 & 20 & 76.10 & 7.09 & 210.86 & 28642.68 & 135.84 & 86.11 & 136.84 & 0.977 \\
\hline $646 \mathrm{~A}$ & $9 \mathrm{H}$ & 2 & 62 & 76.52 & 6.27 & 142.86 & 19193.46 & 134.35 & 47.28 & 83.00 & 0.978 \\
\hline $646 A$ & $9 \mathrm{H}$ & 2 & 84 & 76.74 & 7.85 & 111.22 & 14310.57 & 128.66 & 41.36 & 62.74 & 0.976 \\
\hline $646 \mathrm{~A}$ & $9 \mathrm{H}$ & 2 & 101 & 76.91 & 7.16 & 120.89 & 15889.50 & 131.44 & 41.61 & 68.20 & 0.977 \\
\hline $646 \mathrm{~A}$ & $9 \mathrm{H}$ & 2 & 120 & 77.10 & 6.88 & 119.97 & 15628.29 & 130.27 & 42.87 & 85.63 & 0.973 \\
\hline $646 \mathrm{~A}$ & $9 \mathrm{H}$ & 2 & 140 & 77.30 & 7.25 & 111.07 & 12558.48 & 113.07 & 37.76 & 71.10 & 0.973 \\
\hline $646 \mathrm{~A}$ & $9 \mathrm{H}$ & 2 & 140 & 77.30 & 7.68 & 114.67 & 13023.29 & 113.57 & 41.47 & 80.13 & 0.970 \\
\hline $646 \mathrm{~A}$ & $9 \mathrm{H}$ & 3 & 0 & 77.40 & 7.79 & 114.82 & 12355.75 & 107.61 & 41.42 & 77.55 & 0.971 \\
\hline $646 \mathrm{~A}$ & $9 \mathrm{H}$ & 3 & 23 & 77.63 & 7.28 & 173.95 & 13854.30 & 79.65 & 45.77 & 77.19 & 0.975 \\
\hline $646 \mathrm{~A}$ & $9 \mathrm{H}$ & 3 & 40 & 77.80 & 7.34 & 137.95 & 17099.12 & 123.95 & 56.58 & 85.00 & 0.978 \\
\hline $646 \mathrm{~A}$ & $9 \mathrm{H}$ & 3 & 60 & 78.00 & 7.74 & 202.24 & 17518.31 & 86.62 & 64.67 & 99.69 & 0.976 \\
\hline $646 \mathrm{~A}$ & $9 \mathrm{H}$ & 3 & 85 & 78.25 & 7.49 & 107.85 & 11747.99 & 108.93 & 32.86 & 56.07 & 0.974 \\
\hline $646 \mathrm{~A}$ & $9 \mathrm{H}$ & 3 & 141 & 78.81 & 7.69 & 167.12 & 16492.74 & 98.69 & 62.78 & 135.51 & 0.967 \\
\hline $646 \mathrm{~A}$ & $9 \mathrm{H}$ & $3:$ & 141 & 78.81 & 8.00 & 173.84 & 16785.49 & 96.56 & 66.69 & 147.46 & 0.965 \\
\hline $646 \mathrm{~A}$ & $9 \mathrm{H}$ & 4 & 5 & 78.95 & 7.57 & 168.44 & 16657.76 & 98.89 & 61.06 & 127.12 & 0.968 \\
\hline $646 \mathrm{~A}$ & $9 \mathrm{H}$ & 4 & 20 & 79.10 & 8.52 & 187.26 & 16991.94 & 90.74 & 79.04 & 120.51 & 0.974 \\
\hline $646 A$ & $9 \mathrm{H}$ & 4 & 41 & 79.31 & 7.44 & 158.55 & 15836.91 & 99.88 & 57.48 & 78.33 & 0.980 \\
\hline $646 \mathrm{~A}$ & $9 \mathrm{H}$ & 4 & 61 & 79.51 & 8.10 & 145.48 & 15855.98 & 108.99 & 62.92 & 98.21 & 0.975 \\
\hline $646 \mathrm{~A}$ & $9 \mathrm{H}$ & 4 & 84 & 79.74 & 7.23 & 138.14 & 17254.15 & 124.90 & 51.41 & 73.92 & 0.979 \\
\hline $646 A$ & $9 \mathrm{H}$ & 4 & 101 & 79.91 & 7.42 & 111.74 & 15950.71 & 142.74 & 44.19 & 55.49 & 0.981 \\
\hline $646 A$ & $9 \mathrm{H}$ & 4 & 120 & 80.10 & 4.79 & 110.15 & 14769.83 & 134.08 & 25.13 & 53.67 & 0.980 \\
\hline $646 A$ & $9 \mathrm{H}$ & 4 & 120 & 80.10 & 7.25 & 117.66 & 15610.81 & 132.68 & 43.16 & 53.81 & 0.982 \\
\hline $646 A$ & $9 \mathrm{H}$ & 5 & 0 & 80.40 & 7.72 & 141.74 & 18137.64 & 127.97 & 48.30 & 44.86 & 0.986 \\
\hline $646 A$ & $9 \mathrm{H}$ & 5 & 33 & 80.73 & 7.17 & 153.66 & 20821.51 & 135.50 & 59.57 & 86.90 & 0.979 \\
\hline $646 \mathrm{~A}$ & $9 \mathrm{H}$ & 5 & 56 & 80.96 & 7.15 & 123.87 & 17410.83 & 140.56 & 49.70 & 104.56 & 0.970 \\
\hline $646 \mathrm{~A}$ & $9 \mathrm{H}$ & 5 & 77 & 81.17 & 6.80 & 123.60 & 17374.56 & 140.58 & 46.86 & 102.99 & 0.970 \\
\hline $646 \mathrm{~A}$ & $10 \mathrm{H}$ & 1 & 0 & 84.10 & 7.57 & 103.39 & 11346.80 & 109.75 & 37.98 & 35.90 & 0.986 \\
\hline $646 \mathrm{~A}$ & $10 \mathrm{H}$ & 2 & 40 & 85.60 & 7.03 & 118.66 & 9771.84 & 82.35 & 27.10 & 23.22 & 0.988 \\
\hline $646 \mathrm{~A}$ & $10 \mathrm{H}$ & 2 & 80 & 86.06 & 7.10 & 132.17 & 14651.79 & 110.85 & 49.75 & 60.35 & 0.983 \\
\hline $646 A$ & $10 \mathrm{H}$ & 2 & 100 & 86.20 & 7.08 & 141.24 & 15455.53 & 109.43 & 54.14 & 66.81 & 0.983 \\
\hline $646 A$ & $10 \mathrm{H}$ & 2 & 120 & 86.40 & 6.94 & 129.07 & 12463.41 & 96.57 & 39.80 & 59.16 & 0.979 \\
\hline $646 A$ & $10 \mathrm{H}$ & 2 & 146 & 86.51 & 7.17 & 113.54 & 11348.44 & 99.95 & 42.13 & 123.54 & 0.958 \\
\hline $646 \mathrm{~A}$ & $10 \mathrm{H}$ & 2 & 0 & 86.60 & 7.61 & 122.82 & 14142.49 & 115.15 & 50.65 & 105.88 & 0.968 \\
\hline $646 A$ & $10 \mathrm{H}$ & 2 & 46 & 86.80 & 7.14 & 121.05 & 12178.89 & 100.61 & 35.64 & 47.36 & 0.981 \\
\hline $646 \mathrm{~A}$ & $10 \mathrm{H}$ & 2 & 91 & 87.03 & 6.66 & 160.71 & 16553.49 & 103.00 & 56.09 & 104.67 & 0.975 \\
\hline $646 A$ & $10 \mathrm{H}$ & 3 & 0 & 87.10 & 6.77 & 156.43 & 15292.86 & 97.76 & 59.67 & 148.18 & 0.966 \\
\hline $646 \mathrm{~A}$ & $10 \mathrm{H}$ & 3 & 46 & 87.56 & 5.79 & 183.78 & 17963.96 & 97.75 & 60.01 & 156.06 & 0.970 \\
\hline $646 \mathrm{~A}$ & $10 \mathrm{H}$ & 3 & 134 & 88.44 & 4.62 & 178.92 & 20110.29 & 112.40 & 37.27 & 102.21 & 0.975 \\
\hline $646 \mathrm{~A}$ & $10 \mathrm{H}$ & 3 & 134 & 88.44 & 7.23 & 166.98 & 19633.78 & 117.58 & 60.62 & 105.87 & 0.975 \\
\hline $646 \mathrm{~A}$ & $10 \mathrm{H}$ & 4 & 3 & 88.63 & 7.77 & 126.60 & 14954.76 & 118.13 & 46.54 & 49.88 & 0.983 \\
\hline $646 \mathrm{~A}$ & $10 \mathrm{H}$ & 4 & 46 & 89.06 & 7.43 & 197.83 & 21997.10 & 111.19 & 71.35 & 32.54 & 0.993 \\
\hline $646 \mathrm{~A}$ & $10 \mathrm{H}$ & 4 & 94 & 89.54 & 7.17 & 179.77 & 19812.45 & 110.21 & 58.73 & 27.50 & 0.993 \\
\hline $646 \mathrm{~A}$ & $10 \mathrm{H}$ & 4 & 138 & 89.98 & 7.55 & 152.42 & 15774.24 & 103.49 & 47.38 & 19.53 & 0.994 \\
\hline $646 \mathrm{~A}$ & $10 \mathrm{H}$ & 4 & 138 & 89.98 & 7.75 & 151.56 & 16797.70 & 110.83 & 51.12 & 21.25 & 0.994 \\
\hline $646 \mathrm{~A}$ & $10 \mathrm{H}$ & 5 & 0 & 90.10 & 6.91 & 194.71 & 23374.11 & 120.04 & 70.83 & 47.87 & 0.991 \\
\hline $646 \mathrm{~A}$ & $10 \mathrm{H}$ & 5 & 26 & 90.36 & 6.79 & 167.26 & 20484.60 & 122.47 & 57.57 & 45.71 & 0.989 \\
\hline $646 \mathrm{~A}$ & $10 \mathrm{H}$ & 5 & 69 & 90.79 & 7.21 & 165.01 & 17684.82 & 107.18 & 55.39 & 44.69 & 0.988 \\
\hline $646 A$ & $10 \mathrm{H}$ & 5 & 146 & 91.56 & 8.09 & 197.84 & 19315.37 & 97.63 & 72.33 & 29.23 & 0.993 \\
\hline $646 \mathrm{~A}$ & $10 \mathrm{H}$ & 5 & 146 & 91.56 & 8.45 & 189.11 & 18658.08 & 98.66 & 72.36 & 30.35 & 0.993 \\
\hline $646 \mathrm{~A}$ & $10 \mathrm{H}$ & 6 & 5 & 91.65 & 6.28 & 118.23 & 15131.06 & 127.98 & 33.22 & 24.93 & 0.991 \\
\hline $646 \mathrm{~A}$ & $10 \mathrm{H}$ & 6 & 34 & 91.94 & 7.54 & 156.62 & 17121.07 & 109.32 & 54.26 & 36.34 & 0.990 \\
\hline $646 \mathrm{~A}$ & $10 \mathrm{H}$ & 6 & 109 & 92.69 & 6.54 & 189.59 & 21354.72 & 112.63 & 60.66 & 109.66 & 0.976 \\
\hline $646 \mathrm{~A}$ & $10 \mathrm{H}$ & 6 & 129 & 92.89 & 7.30 & 164.87 & 15240.87 & 92.44 & 45.91 & 30.71 & 0.990 \\
\hline $646 \mathrm{~A}$ & $11 \mathrm{H}$ & 1 & 11 & 93.91 & 7.44 & 120.73 & 15719.90 & 130.21 & 45.00 & 45.74 & 0.985 \\
\hline
\end{tabular}




\begin{tabular}{|c|c|c|c|c|c|c|c|c|c|c|c|}
\hline \multicolumn{4}{|c|}{$\begin{array}{l}\text { Core, section } \\
\text { depth }(\mathrm{cm})\end{array}$} & \multirow{2}{*}{$\begin{array}{c}\begin{array}{c}\text { Depth } \\
\text { (mbsf) }\end{array} \\
94.10\end{array}$} & \multirow{2}{*}{$\begin{array}{c}\begin{array}{c}\text { Mass } \\
(\mathrm{g})\end{array} \\
7.70\end{array}$} & \multirow{2}{*}{$\frac{\begin{array}{c}X \\
\left(10^{-6} \mathrm{~m}^{3} / \mathrm{kg}\right)\end{array}}{126.12}$} & \multirow{2}{*}{$\begin{array}{c}\begin{array}{c}X_{\mathrm{ARM}} \\
\left(10^{-6} \mathrm{~m}^{3} / \mathrm{kg}\right)\end{array} \\
16221.04\end{array}$} & \multirow{2}{*}{$\begin{array}{l}X_{\text {ARM }} / X \\
128.62\end{array}$} & \multirow{2}{*}{$\begin{array}{c}\begin{array}{c}\text { SIRM } \\
(\mathrm{mA} / \mathrm{m} \cdot \mathrm{kg})\end{array} \\
48.05\end{array}$} & \multirow{2}{*}{$\begin{array}{c}\begin{array}{c}\text { HIRM } \\
(\mathrm{mA} / \mathrm{m} \cdot \mathrm{kg})\end{array} \\
45.96\end{array}$} & \multirow{2}{*}{$\frac{S}{0.985}$} \\
\hline $646 \mathrm{~A}$ & $11 \mathrm{H}$ & 1 & 30 & & & & & & & & \\
\hline $646 \mathrm{~A}$ & $11 \mathrm{H}$ & 1 & 52 & 94.32 & 7.13 & 164.92 & 18085.69 & 109.66 & 75.00 & 105.01 & 0.980 \\
\hline $646 \mathrm{~A}$ & $11 \mathrm{H}$ & 1 & 80 & 94.60 & 7.05 & 202.79 & 19383.98 & 95.59 & 71.27 & 76.91 & 0.985 \\
\hline $646 \mathrm{~A}$ & $11 \mathrm{H}$ & 1. & 120 & 95.00 & 6.41 & 182.27 & 19127.03 & 104.94 & 58.85 & 92.20 & 0.980 \\
\hline $646 \mathrm{~A}$ & $11 \mathrm{H}$ & 2 & 0 & 95.30 & 6.94 & 115.85 & 14642.74 & 126.39 & 36.14 & 36.37 & 0.986 \\
\hline $646 A$ & $11 \mathrm{H}$ & 2 & 29 & 95.59 & 7.55 & 139.27 & 16912.38 & 121.43 & 53.64 & 79.65 & 0.978 \\
\hline $646 A$ & $11 \mathrm{H}$ & 2 & 80 & 96.10 & 7.68 & 129.06 & 16460.56 & 127.54 & 43.68 & 36.32 & 0.987 \\
\hline $646 \mathrm{~A}$ & $11 \mathrm{H}$ & 2 & 98 & 96.28 & 8.12 & 189.22 & 17756.27 & 93.84 & 56.26 & 27.60 & 0.992 \\
\hline $646 A$ & $11 \mathrm{H}$ & 2 & 131 & 96.61 & 8.17 & 100.72 & 8443.69 & 83.84 & 27.14 & 20.84 & 0.987 \\
\hline $646 \mathrm{~A}$ & $11 \mathrm{H}$ & 2 & 131 & 96.61 & 7.50 & 94.47 & 8966.83 & 94.91 & 25.71 & 7.96 & 0.995 \\
\hline $646 \mathrm{~A}$ & $11 \mathrm{H}$ & 3 & 3 & 96.83 & 7.33 & 193.50 & 18742.76 & 96.86 & 71.66 & 156.41 & 0.968 \\
\hline $646 \mathrm{~A}$ & $11 \mathrm{H}$ & 3 & 35 & 97.15 & 6.92 & 161.76 & 18512.72 & 114.45 & 59.50 & 160.72 & 0.963 \\
\hline $646 \mathrm{~A}$ & $11 \mathrm{H}$ & 3 & 68 & 97.48 & 7.06 & 161.39 & 16041.79 & 99.39 & 60.14 & 147.89 & 0.965 \\
\hline $646 \mathrm{~A}$ & $11 \mathrm{H}$ & 3 & 116 & 97.96 & 4.20 & 201.30 & 16795.59 & 83. & 41 . & & \\
\hline $646 \mathrm{~A}$ & $11 \mathrm{H}$ & 3 & 116 & 97.96 & 4.51 & 211.14 & 18178.48 & 86.09 & 44.64 & 205.09 & 0.959 \\
\hline $646 \mathrm{~A}$ & $11 \mathrm{H}$ & 4 & 0 & 98.30 & 6.48 & 119.62 & 13024.23 & 108.88 & 38.18 & 111.95 & 0.962 \\
\hline $646 \mathrm{~A}$ & $11 \mathrm{H}$ & 4 & 28 & 98.58 & 6.87 & 132.03 & 15836.46 & 119.95 & 44.27 & 106.32 & 0.967 \\
\hline $646 \mathrm{~A}$ & $11 \mathrm{H}$ & 4 & 75 & 99.05 & 7.24 & 127.71 & 15906.88 & 124.55 & 48.97 & 123.66 & 0.963 \\
\hline $646 \mathrm{~A}$ & $11 \mathrm{H}$ & 4 & 116 & 99.46 & 7.71 & 223.56 & 25889.83 & 115.81 & 98.19 & 340 . & 0.947 \\
\hline $646 \mathrm{~A}$ & $11 \mathrm{H}$ & 4 & 139 & 99.69 & 4.91 & 220.30 & 25298.85 & 114.84 & 56.93 & 201.99 & 0.965 \\
\hline $646 \mathrm{~A}$ & $11 \mathrm{H}$ & 4 & 139 & 99.69 & 6.21 & 194.21 & 24272.73 & 124.98 & 70.44 & 181.69 & 0.968 \\
\hline $646 \mathrm{~A}$ & $11 \mathrm{H}$ & 5 & 0 & 99.80 & 7.28 & 189.13 & 21167.76 & 111.92 & 75.90 & 87.27 & 0.983 \\
\hline $646 \mathrm{~A}$ & $11 \mathrm{H}$ & 5 & 26 & 100.06 & 7.46 & 142.97 & 19731.20 & 138.01 & 53.62 & 113.62 & 0.968 \\
\hline $646 \mathrm{~A}$ & $11 \mathrm{H}$ & 5 & 60 & 100.40 & 7.82 & 127.88 & 14619.13 & 114.32 & 45.76 & 101.22 & 0.965 \\
\hline $646 \mathrm{~A}$ & $11 \mathrm{H}$ & 5 & 113 & 100.93 & 7.22 & 123.89 & 13129.53 & 105.98 & 44.08 & 161.28 & 0.947 \\
\hline $646 \mathrm{~A}$ & $11 \mathrm{H}$ & 5 & 113 & 100.93 & 5.03 & 129.62 & 13899.77 & 107.23 & 33.72 & 227.71 & 0.932 \\
\hline $646 \mathrm{~A}$ & $11 \mathrm{H}$ & 6 & 0 & 101.30 & 7.85 & 198.12 & 17756.78 & 89.62 & 83.41 & 278.42 & 0.948 \\
\hline $646 \mathrm{~A}$ & $11 \mathrm{H}$ & 6 & 85 & 102.15 & 8.74 & 228.40 & 17340.71 & 75.92 & 98.24 & 334.59 & 0.940 \\
\hline $646 \mathrm{~A}$ & $11 \mathrm{H}$ & 6 & 117 & 102.47 & 7.81 & 153.94 & 15230.28 & 98.94 & 58.85 & 113.15 & 0.970 \\
\hline $646 \mathrm{~B}$ & $1 \mathrm{H}$ & 1 & 0 & 0.00 & 6.67 & 152.37 & 10130.64 & 66.49 & 47.13 & 98.04 & 0.972 \\
\hline $646 \mathrm{~B}$ & $1 \mathrm{H}$ & 1 & 10 & 0.10 & 6.86 & 118.12 & 11004.92 & 93.17 & 38.23 & 104.75 & 0.962 \\
\hline $646 \mathrm{~B}$ & $1 \mathrm{H}$ & 1 & 28 & 0.28 & 3.40 & 121.56 & 10214.68 & 84.03 & 16.54 & 66.44 & 0.973 \\
\hline $646 \mathrm{~B}$ & $1 \mathrm{H}$ & 1 & 50 & 0.50 & 7.51 & 110.24 & 9951.56 & 90.27 & 37.45 & 51.18 & 0.979 \\
\hline $646 \mathrm{~B}$ & $1 \mathrm{H}$ & 1 & 70 & 0.70 & 6.52 & 123.89 & 12736.03 & 102.80 & 36.87 & 77.55 & 0.973 \\
\hline $646 \mathrm{~B}$ & $1 \mathrm{H}$ & 1 & 90 & 0.90 & 6.10 & 93.29 & 13494.73 & 144.65 & 33.00 & 94.80 & 0.965 \\
\hline $646 \mathrm{~B}$ & $1 \mathrm{H}$ & 1 & 110 & 1.10 & 6.44 & 87.00 & 9713.55 & 111.65 & 40.20 & 113.31 & 0.964 \\
\hline $646 \mathrm{~B}$ & $1 \mathrm{H}$ & 1 & 130 & 1.30 & 7.02 & 119.19 & 10114.50 & 84.86 & 51.63 & 99.25 & 0.973 \\
\hline $646 \mathrm{~B}$ & $1 H$ & 1 & 145 & 1.45 & 6.94 & 90.51 & 11360.11 & 125.51 & 43.02 & 106.83 & 0.966 \\
\hline $646 \mathrm{~B}$ & $1 \mathrm{H}$ & 1 & 145 & 1.45 & 6.95 & 87.13 & 10708.04 & 122.90 & 40.14 & 110.10 & 0.962 \\
\hline $646 \mathrm{~B}$ & $1 \mathrm{H}$ & 2 & 0 & 1.50 & 7.34 & 93.96 & 11068.63 & 117.80 & 45.53 & 67.14 & 0.978 \\
\hline $646 \mathrm{~B}$ & $1 \mathrm{H}$ & 2 & 20 & 1.70 & 7.23 & 89.66 & 11391.15 & 127.05 & 43.71 & 105.16 & 0.965 \\
\hline $646 \mathrm{~B}$ & $1 \mathrm{H}$ & 2 & 40 & 1.90 & 5.74 & 98.93 & 11118.53 & 112.39 & 24.89 & 81.23 & 0.963 \\
\hline $646 \mathrm{~B}$ & $1 \mathrm{H}$ & 2 & 64 & 2.14 & 8.06 & 120.95 & 8120.94 & 67.14 & 33.58 & 31.37 & 0.985 \\
\hline $646 \mathrm{~B}$ & $1 \mathrm{H}$ & 2 & 80 & 2.30 & 6.82 & 129.86 & 13672.84 & 105.29 & 43.29 & 60.24 & 0.981 \\
\hline $646 \mathrm{~B}$ & $1 \mathrm{H}$ & 2 & 100 & 2.50 & 6.12 & 132.61 & 14509.64 & 109.42 & 40.48 & 74.09 & 0.978 \\
\hline $646 \mathrm{~B}$ & $1 \mathrm{H}$ & 2 & 140 & 2.90 & 7.48 & 125.80 & 12605.31 & 100.20 & 42.98 & 43.22 & 0.985 \\
\hline $646 \mathrm{~B}$ & $1 \mathrm{H}$ & 2 & 140 & 2.90 & 6.53 & 122.17 & 11992.20 & 98.16 & 36.18 & 44.82 & 0.984 \\
\hline $646 \mathrm{~B}$ & $1 \mathrm{H}$ & 3 & 0 & 3.00 & 7.06 & 111.04 & 11562.95 & 104.14 & 36.64 & 61.44 & 0.976 \\
\hline $646 \mathrm{~B}$ & $1 \mathrm{H}$ & 3 & 10 & 3.10 & 6.13 & 97.55 & 11236.44 & 115.18 & 30.78 & 66.08 & 0.974 \\
\hline $646 \mathrm{~B}$ & $1 \mathrm{H}$ & 3 & 30 & 3.30 & 5.67 & 90.18 & 9961.85 & 110.47 & 25.14 & 36.01 & 0.984 \\
\hline $646 \mathrm{~B}$ & $1 \mathrm{H}$ & 3 & 51 & 3.51 & 7.29 & 99.43 & 9965.12 & 100.22 & 34.61 & 56.34 & 0.976 \\
\hline $646 \mathrm{~B}$ & $1 \mathrm{H}$ & 3 & 72 & 3.72 & 6.88 & 74.68 & 7949.63 & 106.44 & 23.49 & 43.01 & 0.975 \\
\hline $646 \mathrm{~B}$ & $1 \mathrm{H}$ & 3 & 90 & 3.90 & 7.41 & 92.91 & 9247.66 & 99.54 & 30.01 & 4.01 & 0.998 \\
\hline $646 \mathrm{~B}$ & $1 \mathrm{H}$ & 3 & 110 & 4.10 & 7.60 & 124.64 & 11657.13 & 93.53 & 41.49 & 85.43 & 0.969 \\
\hline $646 \mathrm{~B}$ & $1 \mathrm{H}$ & 3 & 130 & 4.30 & 6.19 & 100.87 & 11701.89 & 116.01 & 34.05 & 81.65 & 0.970 \\
\hline $646 \mathrm{~B}$ & $1 \mathrm{H}$ & 3 & 130 & 4.30 & 6.33 & 110.74 & 12738.85 & 115.03 & 37.79 & 90.67 & 0.970 \\
\hline $646 \mathrm{~B}$ & $1 \mathrm{H}$ & $3:$ & 148 & 4.48 & 7.01 & 127.24 & 13444.72 & 105.66 & 47.62 & 47.95 & 0.986 \\
\hline
\end{tabular}


Appendix B (continued)

\begin{tabular}{|c|c|c|c|c|c|c|c|c|c|c|c|}
\hline \multicolumn{4}{|c|}{$\begin{array}{l}\text { Core, section } \\
\text { depth }(\mathrm{cm})\end{array}$} & \multirow{2}{*}{$\begin{array}{l}\begin{array}{l}\text { Depth } \\
\text { (mbsf) }\end{array} \\
4.50\end{array}$} & \multirow{2}{*}{$\begin{array}{c}\begin{array}{c}\text { Mass } \\
(\mathrm{g})\end{array} \\
6.26\end{array}$} & \multirow{2}{*}{$\frac{\begin{array}{c}X \\
\left(10^{-6} \mathrm{~m}^{3} / \mathrm{kg}\right)\end{array}}{114.19}$} & \multirow{2}{*}{$\begin{array}{c}\begin{array}{c}X_{\mathrm{ARM}} \\
\left(10^{-6} \mathrm{~m}^{3} / \mathrm{kg}\right)\end{array} \\
10989.32\end{array}$} & \multirow{2}{*}{$\frac{X_{\mathrm{ARM}} / X}{96.24}$} & \multirow{2}{*}{$\begin{array}{c}\begin{array}{c}\text { SIRM } \\
(\mathrm{mA} / \mathrm{m} \cdot \mathrm{kg})\end{array} \\
37.80\end{array}$} & \multirow{2}{*}{$\begin{array}{c}\begin{array}{c}\text { HIRM } \\
(\mathrm{mA} / \mathrm{m} \cdot \mathrm{kg})\end{array} \\
89.32\end{array}$} & \multirow{2}{*}{$\frac{S}{0.970}$} \\
\hline 646B & $1 \mathrm{H}$ & 4 & 0 & & & & & & & & \\
\hline $646 B$ & $1 \mathrm{H}$ & 4 & 20 & 4.70 & 6.73 & 114.80 & 12103.98 & 105.43 & 41.22 & 32.03 & 0.990 \\
\hline $646 \mathrm{~B}$ & $1 \mathrm{H}$ & 4 & 40 & 4.90 & 7.39 & 122.06 & 11890.98 & 97.42 & 48.58 & 37.38 & 0.989 \\
\hline $646 B$ & IH & 4 & 60 & 5.10 & 7.02 & 123.84 & 12340.01 & 99.65 & 42.68 & 42.95 & 0.986 \\
\hline $646 \mathrm{~B}$ & $1 \mathrm{H}$ & 4 & 82 & 5.32 & 6.66 & 90.54 & 9885.01 & 109.18 & 37.61 & 85.44 & 0.970 \\
\hline $646 \mathrm{~B}$ & $1 \mathrm{H}$ & 41 & 116 & 5.66 & 6.99 & 116.28 & 11973.31 & 102.97 & 48.20 & 63.58 & 0.982 \\
\hline $646 \mathrm{~B}$ & $1 \mathrm{H}$ & 41 & 116 & 5.66 & 7.19 & 112.87 & 11518.13 & 102.04 & 48.36 & 62.09 & 0.982 \\
\hline $646 B$ & $1 \mathrm{H}$ & 41 & 138 & 5.88 & 7.36 & 145.26 & 13804.93 & 95.04 & 61.75 & 72.97 & 0.983 \\
\hline $646 B$ & $1 \mathrm{H}$ & 5 & 10 & 6.10 & 6.60 & 128.67 & 13558.51 & 105.37 & 47.19 & 67.61 & 0.981 \\
\hline 646B & $1 \mathrm{H}$ & 5 & 30 & 6.30 & 5.88 & 142.08 & 14247.04 & 100.28 & 46.00 & 77.99 & 0.980 \\
\hline $646 B$ & $1 \mathrm{H}$ & 5 & 50 & 6.50 & 5.83 & 152.78 & 14515.55 & 95.01 & 48.74 & 83.47 & 0.980 \\
\hline $646 \mathrm{~B}$ & $1 \mathrm{H}$ & 5 & 94 & 6.94 & 6.28 & 148.43 & 15010.96 & 101.13 & 52.71 & 94.55 & 0.977 \\
\hline $646 \mathrm{~B}$ & $1 \mathrm{H}$ & 51 & 110 & 7.10 & 7.33 & 155.28 & 15983.22 & 102.93 & 62.95 & 88.45 & 0.979 \\
\hline $646 \mathrm{~B}$ & $1 \mathrm{H}$ & 51 & 130 & 7.30 & 6.49 & 146.15 & 15381.80 & 105.25 & 52.68 & 91.49 & 0.977 \\
\hline $646 \mathrm{~B}$ & $1 \mathrm{H}$ & 51 & 130 & 7.30 & 6.04 & 147.26 & 15379.67 & 104.44 & 48.12 & 92.74 & 0.977 \\
\hline $646 \mathrm{~B}$ & $1 \mathrm{H}$ & 51 & 148 & 7.48 & 6.08 & 161.79 & 15651.65 & 96.74 & 55.06 & 181.42 & 0.960 \\
\hline $646 \mathrm{~B}$ & $1 \mathrm{H}$ & 6 & 20 & 7.70 & 6.91 & 171.26 & 16407.11 & 95.80 & 64.06 & 176.41 & 0.962 \\
\hline $646 \mathrm{~B}$ & $1 \mathrm{H}$ & 6 & 36 & 7.86 & 6.59 & 158.61 & 16145.60 & 101.80 & 57.91 & 180.97 & 0.959 \\
\hline $646 \mathrm{~B}$ & $1 \mathrm{H}$ & 6 & 60 & 8.10 & 6.38 & 124.25 & 13351.04 & 107.45 & 45.41 & 161.24 & 0.955 \\
\hline $646 \mathrm{~B}$ & $1 \mathrm{H}$ & 6 & 80 & 8.30 & 6.57 & 96.18 & 10609.17 & 110.30 & 39.26 & 163.73 & 0.945 \\
\hline $646 \mathrm{~B}$ & $1 \mathrm{H}$ & 61 & 100 & 8.50 & 5.98 & 118.91 & 11795.39 & 99.20 & 37.95 & 161.45 & 0.949 \\
\hline $646 \mathrm{~B}$ & $1 \mathrm{H}$ & 61 & 120 & 8.70 & 6.52 & 127.55 & 12510.60 & 98.08 & 41.31 & 115.58 & 0.964 \\
\hline $646 \mathrm{~B}$ & $1 \mathrm{H}$ & 61 & 120 & 8.70 & 6.41 & 123.86 & 12298.43 & 99.29 & 39.29 & 113.29 & 0.963 \\
\hline $646 \mathrm{~B}$ & $1 \mathrm{H}$ & 61 & 137 & 8.87 & 6.03 & 144.38 & 14778.29 & 102.36 & 46.82 & 162.48 & 0.958 \\
\hline $646 \mathrm{~B}$ & $2 \mathrm{H}$ & 1 & 0 & 9.00 & 7.09 & 155.93 & 10384.88 & 66.60 & 52.18 & 84.14 & 0.977 \\
\hline $646 \mathrm{~B}$ & $2 \mathrm{H}$ & 1 & 20 & 9.20 & 6.64 & 115.60 & 10576.89 & 91.50 & 43.81 & 2.90 & 0.999 \\
\hline $646 \mathrm{~B}$ & $2 \mathrm{H}$ & 1 & 40 & 9.40 & 6.90 & 119.98 & 10497.00 & 87.49 & 46.68 & 70.09 & 0.979 \\
\hline $646 \mathrm{~B}$ & $2 \mathrm{H}$ & 1 & 60 & 9.60 & 6.98 & 127.61 & 10289.94 & 80.64 & 46.25 & 51.07 & 0.985 \\
\hline $646 \mathrm{~B}$ & $2 \mathrm{H}$ & 1 & 80 & 9.80 & 6.88 & 140.05 & 13012.51 & 92.91 & 54.32 & 45.40 & 0.989 \\
\hline $646 \mathrm{~B}$ & $2 \mathrm{H}$ & 1 & 102 & 10.02 & 5.30 & 120.41 & 12674.22 & 105.26 & 34.57 & 142.52 & 0.956 \\
\hline $646 \mathrm{~B}$ & $2 \mathrm{H}$ & 11 & 120 & 10.20 & 5.81 & 131.03 & 13514.65 & 103.14 & 40.39 & 138.93 & 0.960 \\
\hline $646 \mathrm{~B}$ & $2 \mathrm{H}$ & 11 & 145 & 10.45 & 6.05 & 148.26 & 15660.95 & 105.63 & 49.87 & 176.83 & 0.957 \\
\hline $646 \mathrm{~B}$ & $2 \mathrm{H}$ & 1 & 145 & 10.45 & 6.78 & 147.86 & 15241.21 & 103.08 & 56.75 & 175.83 & 0.958 \\
\hline $646 \mathrm{~B}$ & $2 \mathrm{H}$ & 2 & 0 & 10.50 & 5.82 & 162.11 & 16165.02 & 99.72 & 52.62 & 205.46 & 0.955 \\
\hline $646 \mathrm{~B}$ & $2 \mathrm{H}$ & 2 & 20 & 10.70 & 7.14 & 164.86 & 16290.84 & 98.81 & 66.25 & 206.94 & 0.955 \\
\hline $646 \mathrm{~B}$ & $2 \mathrm{H}$ & 2 & 40 & 10.90 & 6.35 & 163.81 & 15748.82 & 96.14 & 56.70 & 204.60 & 0.954 \\
\hline $646 \mathrm{~B}$ & $2 \mathrm{H}$ & 2 & 60 & 11.10 & 6.54 & 134.27 & 14535.02 & 108.25 & 51.31 & 198.99 & 0.949 \\
\hline $646 \mathrm{~B}$ & $2 \mathrm{H}$ & 2 & 90 & 11.40 & 7.14 & 102.05 & 10814.58 & 105.97 & 45.68 & 184.51 & 0.942 \\
\hline $646 \mathrm{~B}$ & $2 \mathrm{H}$ & 2 & 110 & 11.60 & 5.59 & 101.58 & 10801.32 & 106.33 & 34.32 & 191.26 & 0.938 \\
\hline $646 \mathrm{~B}$ & $2 \mathrm{H}$ & 2 & 130 & 11.80 & 7.10 & 125.63 & 12145.41 & 96.68 & 46.67 & 144.71 & 0.956 \\
\hline $646 \mathrm{~B}$ & $2 \mathrm{H}$ & 2 & 148 & 11.98 & 7.07 & 128.83 & 13355.14 & 103.67 & 45.91 & 129.43 & 0.960 \\
\hline $646 \mathrm{~B}$ & $2 \mathrm{H}$ & 3 & 10 & 12.10 & 6.21 & 134.53 & 14111.01 & 104.89 & 43.45 & 156.13 & 0.955 \\
\hline $646 \mathrm{~B}$ & $2 \mathrm{H}$ & 3 & 30 & 12.30 & 6.30 & 133.01 & 14110.63 & 106.09 & 45.91 & 176.78 & 0.951 \\
\hline $646 \mathrm{~B}$ & $2 \mathrm{H}$ & 3 & 50 & 12.50 & 6.72 & 134.79 & 14280.67 & 105.95 & 50.67 & 155.75 & 0.959 \\
\hline $646 \mathrm{~B}$ & $2 \mathrm{H}$ & 3 & 70 & 12.70 & 6.52 & 136.42 & 13500.02 & 98.96 & 45.52 & 188.32 & 0.946 \\
\hline $646 \mathrm{~B}$ & $2 \mathrm{H}$ & 3 & 90 & 12.90 & 7.20 & 114.98 & 12135.15 & 105.54 & 47.72 & 160.32 & 0.952 \\
\hline $646 \mathrm{~B}$ & $2 \mathrm{H}$ & 3 & 110 & 13.10 & 7.27 & 99.02 & 9912.70 & 100.11 & 40.90 & 131.45 & 0.953 \\
\hline $646 \mathrm{~B}$ & $2 \mathrm{H}$ & 3 & 130 & 13.30 & 6.20 & 114.08 & 8546.36 & 74.92 & 33.13 & 121.24 & 0.955 \\
\hline $646 B$ & $2 \mathrm{H}$ & 3 & 130 & 13.30 & 6.77 & 127.85 & 8969.89 & 70.16 & 39.28 & 118.08 & 0.959 \\
\hline $646 \mathrm{~B}$ & $2 \mathrm{H}$ & 4 & 8 & 13.58 & 7.30 & 143.87 & 9685.24 & 67.32 & 47.81 & 131.63 & 0.960 \\
\hline $646 B$ & $2 \mathrm{H}$ & 4 & 30 & 13.80 & 7.98 & 161.21 & 10538.12 & 65.37 & 59.10 & 119.98 & 0.968 \\
\hline $646 B$ & $2 \mathrm{H}$ & 4 & 51 & 14.01 & 7.34 & 178.34 & 11586.71 & 64.97 & 59.32 & 137.55 & 0.966 \\
\hline $646 \mathrm{~B}$ & $2 \mathrm{H}$ & 4 & 70 & 14.20 & 7.44 & 156.02 & 12379.94 & 79.35 & 54.01 & 116.60 & 0.968 \\
\hline $646 \mathrm{~B}$ & $2 \mathrm{H}$ & 4 & 91 & 14.40 & 6.79 & 194.83 & 12079.36 & 62.00 & 58.54 & 178.69 & 0.959 \\
\hline $646 \mathrm{~B}$ & $2 \mathrm{H}$ & 4 & 110 & 14.41 & 8.05 & 188.21 & 12033.93 & 63.94 & 67.70 & 157.03 & 0.963 \\
\hline $646 \mathrm{~B}$ & $2 \mathrm{H}$ & 4 & 131 & 14.50 & 7.42 & 190.81 & 12553.16 & 65.79 & 64.58 & 152.29 & 0.965 \\
\hline $646 B$ & $2 \mathrm{H}$ & 4 & 131 & 14.60 & 8.00 & 198.65 & 12898.08 & 64.93 & 72.23 & 137.14 & 0.970 \\
\hline $646 \mathrm{~B}$ & $2 \mathrm{H}$ & 5 & 10 & 14.70 & 7.75 & 159.02 & 12439.46 & 78.23 & 58.77 & 118.33 & 0.969 \\
\hline
\end{tabular}




\begin{tabular}{|c|c|c|c|c|c|c|c|c|c|c|c|}
\hline \multicolumn{4}{|c|}{$\begin{array}{l}\text { Core, section } \\
\text { depth }(\mathrm{cm})\end{array}$} & \multirow{2}{*}{$\begin{array}{r}\begin{array}{r}\text { Depth } \\
\text { (mbsf) }\end{array} \\
14.81\end{array}$} & \multirow{2}{*}{$\begin{array}{c}\begin{array}{c}\text { Mass } \\
(\mathrm{g})\end{array} \\
7.30\end{array}$} & \multirow{2}{*}{$\frac{\begin{array}{c}X \\
\left(10^{-6} \mathrm{~m}^{3} / \mathrm{kg}\right)\end{array}}{182.07}$} & \multirow{2}{*}{$\begin{array}{c}\begin{array}{c}X_{\mathrm{ARM}} \\
\left(10^{-6} \mathrm{~m}^{3} / \mathrm{kg}\right)\end{array} \\
12828.01\end{array}$} & \multirow{2}{*}{$\begin{array}{r}X_{\mathrm{ARM}} / X \\
70.45\end{array}$} & \multirow{2}{*}{$\begin{array}{c}\begin{array}{c}\text { SIRM } \\
(\mathrm{mA} / \mathrm{m} \cdot \mathrm{kg})\end{array} \\
59.21\end{array}$} & \multirow{2}{*}{$\begin{array}{c}\begin{array}{c}\text { HIRM } \\
(\mathrm{mA} / \mathrm{m} \cdot \mathrm{kg})\end{array} \\
163.10\end{array}$} & \multirow{2}{*}{$\frac{S}{0.960}$} \\
\hline $646 B$ & $2 \mathrm{H}$ & 5 & 30 & & & & & & & & \\
\hline $646 \mathrm{~B}$ & $2 \mathrm{H}$ & 5 & 50 & 14.81 & 6.16 & 195.17 & 14606.31 & 74.84 & 50.82 & 175.41 & 0.957 \\
\hline $646 \mathrm{~B}$ & $2 \mathrm{H}$ & 5 & 93 & 14.90 & 7.06 & 158.01 & 13073.87 & 82.74 & 53.71 & 94.72 & 0.975 \\
\hline $646 \mathrm{~B}$ & $2 \mathrm{H}$ & 51 & 130 & 15.10 & 6.59 & 122.39 & 13282.84 & 108.53 & 36.80 & 80.41 & 0.971 \\
\hline $646 B$ & $2 \mathrm{H}$ & 51 & 130 & 15.10 & 6.48 & 124.66 & 13591.49 & 109.03 & 37.59 & 79.68 & 0.973 \\
\hline $646 B$ & $2 \mathrm{H}$ & 6 & 0 & 15.30 & 6.51 & 105.94 & 11875.82 & 112.09 & 30.96 & 58.04 & 0.976 \\
\hline $646 \mathrm{~B}$ & $2 \mathrm{H}$ & 6 & 21 & 15.50 & 7.16 & 131.94 & 14685.51 & 111.30 & 43.21 & 89.53 & 0.970 \\
\hline $646 \mathrm{~B}$ & $2 \mathrm{H}$ & 6 & 41 & 15.50 & 5.88 & 109.60 & 13361.24 & 121.90 & 30.50 & 114.64 & 0.956 \\
\hline $646 B$ & $2 \mathrm{H}$ & 6 & 60 & 15.70 & 5.97 & 132.15 & 14665.67 & 110.98 & 34.49 & 94.93 & 0.967 \\
\hline $646 B$ & $3 \mathrm{H}$ & 1 & 0 & 15.70 & 7.68 & 151.96 & 10964.53 & 72.15 & 53.09 & 94.85 & 0.973 \\
\hline $646 \mathrm{~B}$ & $3 \mathrm{H}$ & 1 & 10 & 15.88 & 7.30 & 140.43 & 11647.79 & 82.94 & 45.99 & 24.84 & 0.992 \\
\hline $646 \mathrm{~B}$ & $3 \mathrm{H}$ & 1 & 30 & 15.90 & 6.31 & 141.56 & 13280.35 & 93.82 & 51.14 & 148.22 & 0.963 \\
\hline $646 \mathrm{~B}$ & $3 \mathrm{H}$ & 1 & 50 & 15.93 & 6.26 & 158.54 & 15122.14 & 95.38 & 52.33 & 165.90 & 0.960 \\
\hline $646 \mathrm{~B}$ & $3 \mathrm{H}$ & 1 & 70 & 16.10 & 6.95 & 126.35 & 13789.27 & 109.13 & 48.13 & 135.48 & 0.961 \\
\hline $646 \mathrm{~B}$ & $3 \mathrm{H}$ & 1 & 110 & 16.30 & 7.34 & 174.07 & 17195.65 & 98.79 & 71.83 & 183.57 & 0.962 \\
\hline $646 B$ & $3 \mathrm{H}$ & 11 & 130 & 16.30 & 7.37 & 131.76 & 13533.92 & 102.71 & 54.60 & 140.09 & 0.962 \\
\hline $646 B$ & $3 \mathrm{H}$ & 11 & 130 & 16.30 & 6.41 & 138.76 & 14144.83 & 101.94 & 49.01 & 147.81 & 0.961 \\
\hline $646 \mathrm{~B}$ & $3 \mathrm{H}$ & 11 & 148 & 16.50 & 6.97 & 129.77 & 13307.39 & 102.54 & 51.28 & 149.62 & 0.959 \\
\hline $646 B$ & $3 \mathrm{H}$ & 2 & 0 & 16.50 & 7.21 & 130.51 & 13515.39 & 103.56 & 55.03 & 157.92 & 0.959 \\
\hline $646 B$ & $3 \mathrm{H}$ & 2 & 20 & 16.71 & 6.35 & 123.85 & 13029.91 & 105.21 & 46.36 & 155.78 & 0.957 \\
\hline $646 B$ & $3 \mathrm{H}$ & 2 & 40 & 16.90 & 6.36 & 120.49 & 12882.81 & 106.92 & 45.75 & 149.81 & 0.958 \\
\hline $646 \mathrm{~B}$ & $3 \mathrm{H}$ & 2 & 60 & 16.91 & 6.50 & 123.12 & 12755.70 & 103.61 & 48.22 & 164.56 & 0.956 \\
\hline $646 B$ & $3 \mathrm{H}$ & 2 & 100 & 17.10 & 7.48 & 139.40 & 13483.70 & 96.73 & 59.79 & 162.85 & 0.959 \\
\hline $646 \mathrm{~B}$ & $3 \mathrm{H}$ & 2 & 120 & 17.10 & 6.58 & 151.21 & 14055.27 & 92.95 & 55.61 & 194.31 & 0.954 \\
\hline $646 B$ & $3 \mathrm{H}$ & 21 & 140 & 17.30 & 6.70 & 160.32 & 14118.17 & 88.06 & 60.30 & 180.82 & 0.960 \\
\hline $646 \mathrm{~B}$ & $3 \mathrm{H}$ & 2 & 140 & 17.30 & 6.40 & 160.57 & 14391.88 & 89.63 & 56.90 & 164.65 & 0.963 \\
\hline $646 B$ & $3 \mathrm{H}$ & 3 & 0 & 17.40 & 5.98 & 136.97 & 12577.10 & 91.82 & 45.48 & 138.64 & 0.964 \\
\hline $646 B$ & $3 \mathrm{H}$ & 3 & 20 & 17.60 & 5.27 & 156.62 & 14174.29 & 90.50 & 44.86 & 143.94 & 0.966 \\
\hline $646 \mathrm{~B}$ & $3 \mathrm{H}$ & 3 & 40 & 17.80 & 6.22 & 160.77 & 15733.01 & 97.86 & 54.70 & 148.33 & 0.966 \\
\hline $646 \mathrm{~B}$ & $3 \mathrm{H}$ & 3 & 60 & 18.00 & 7.36 & 129.21 & 11943.38 & 92.43 & 49.69 & 149.73 & 0.956 \\
\hline $646 \mathrm{~B}$ & $3 \mathrm{H}$ & 3 & 80 & 18.20 & 6.66 & 147.70 & 15625.24 & 105.79 & 52.31 & 132.17 & 0.966 \\
\hline $646 \mathrm{~B}$ & $3 \mathrm{H}$ & 3 & 100 & 18.40 & 6.35 & 167.77 & 16945.16 & 101.00 & 56.17 & 131.28 & 0.970 \\
\hline 646B & $3 \mathrm{H}$ & 3 & 120 & 18.60 & 7.42 & 176.76 & 17106.76 & 96.78 & 70.04 & 166.38 & 0.965 \\
\hline $646 \mathrm{~B}$ & $3 \mathrm{H}$ & 3 & 140 & 18.80 & 7.26 & 152.97 & 16111.55 & 105.33 & 58.53 & 154.40 & 0.962 \\
\hline $646 \mathrm{~B}$ & $3 \mathrm{H}$ & 3 & 140 & 18.80 & 6.12 & 165.86 & 17226.25 & 103.86 & 52.78 & 190.75 & 0.956 \\
\hline 646B & $3 \mathrm{H}$ & 4 & 0 & 18.90 & 6.99 & 120.60 & 13071.44 & 108.39 & 44.92 & 140.17 & 0.956 \\
\hline $646 B$ & $3 \mathrm{H}$ & 4 & 20 & 19.10 & 5.81 & 143.79 & 16029.37 & 111.48 & 45.20 & 158.69 & 0.959 \\
\hline $646 \mathrm{~B}$ & $3 \mathrm{H}$ & 4 & 40 & 19.30 & 6.95 & 127.80 & 14158.20 & 110.79 & 49.89 & 153.26 & 0.957 \\
\hline $646 \mathrm{~B}$ & $3 \mathrm{H}$ & 4 & 60 & 19.50 & 6.66 & 114.50 & 12854.81 & 112.27 & 44.89 & 138.00 & 0.959 \\
\hline $646 \mathrm{~B}$ & $3 \mathrm{H}$ & 4 & 81 & 19.71 & 7.64 & 133.52 & 13569.32 & 101.63 & 54.50 & 134.61 & 0.962 \\
\hline $646 \mathrm{~B}$ & $3 \mathrm{H}$ & $4:$ & 100 & 19.90 & 7.22 & 130.15 & 12503.48 & 96.07 & 49.00 & 136.11 & 0.960 \\
\hline $646 \mathrm{~B}$ & $3 \mathrm{H}$ & 4 & 122 & 20.12 & 7.93 & 195.49 & 13992.09 & 71.57 & 71.52 & 78.27 & 0.983 \\
\hline $646 \mathrm{~B}$ & $3 \mathrm{H}$ & 4 & 140 & 20.30 & 5.40 & 204.73 & 14975.34 & 73.15 & 49.22 & 155.36 & 0.966 \\
\hline $646 \mathrm{~B}$ & $3 \mathrm{H}$ & 4 & 140 & 20.30 & 7.49 & 211.67 & 15252.50 & 72.06 & 74.28 & 171.29 & 0.965 \\
\hline $646 B$ & $3 \mathrm{H}$ & 5 & 0 & 20.40 & 6.59 & 160.90 & 14783.52 & 91.88 & 51.37 & 42.61 & 0.989 \\
\hline $646 \mathrm{~B}$ & $3 \mathrm{H}$ & 5 & 40 & 20.80 & 7.28 & 94.74 & 9337.73 & 98.56 & 32.36 & 36.24 & 0.984 \\
\hline $646 \mathrm{~B}$ & $3 \mathrm{H}$ & 5 & 100 & 21.40 & 7.12 & 133.22 & 13155.31 & 98.75 & 40.56 & 19.93 & 0.993 \\
\hline $646 \mathrm{~B}$ & $3 \mathrm{H}$ & 5 & 118 & 21.58 & 7.32 & 87.70 & 9270.05 & 105.70 & 31.12 & 20.31 & 0.990 \\
\hline $646 B$ & $3 \mathrm{H}$ & 6 & 10 & 22.00 & 7.77 & 59.01 & 6639.70 & 112.51 & 21.34 & 9.39 & 0.993 \\
\hline $646 \mathrm{~B}$ & $3 \mathrm{H}$ & 6 & 30 & 22.20 & 6.68 & 118.29 & 12471.23 & 105.43 & 37.94 & 36.19 & 0.987 \\
\hline $646 \mathrm{~B}$ & $3 \mathrm{H}$ & 6 & 50 & 22.40 & 7.21 & 154.90 & 14567.81 & 94.05 & 54.63 & 50.50 & 0.987 \\
\hline $646 \mathrm{~B}$ & $3 \mathrm{H}$ & 6 & 67 & 22.57 & 6.50 & 126.40 & 12568.81 & 99.44 & 34.50 & 40.96 & 0.985 \\
\hline $646 \mathrm{~B}$ & $3 \mathrm{H}$ & 6 & 95 & 22.85 & 6.66 & 150.72 & 15073.68 & 100.01 & 45.91 & 54.62 & 0.984 \\
\hline $646 \mathrm{~B}$ & $3 \mathrm{H}$ & 67 & 114 & 23.04 & 7.28 & 152.89 & 14420.49 & 94.32 & 45.66 & 37.21 & 0.988 \\
\hline $646 \mathrm{~B}$ & $3 \mathrm{H}$ & 63 & 135 & 23.25 & 6.68 & 142.93 & 13028.65 & 91.15 & 39.28 & 37.95 & 0.987 \\
\hline $646 \mathrm{~B}$ & $3 \mathrm{H}$ & 67 & 135 & 23.25 & 6.39 & 142.54 & 13268.81 & 93.09 & 35.85 & 27.33 & 0.990 \\
\hline $646 \mathrm{~B}$ & $3 \mathrm{H}$ & 7 & 3 & 23.43 & 7.60 & 114.88 & 11129.17 & 96.87 & 40.73 & 58.95 & 0.978 \\
\hline
\end{tabular}


Appendix B (continued)

\begin{tabular}{|c|c|c|c|c|c|c|c|c|c|c|c|}
\hline \multicolumn{4}{|c|}{$\begin{array}{l}\text { Core, section } \\
\text { depth }(\mathrm{cm})\end{array}$} & \multirow{2}{*}{$\begin{array}{r}\begin{array}{r}\text { Depth } \\
\text { (mbsf) }\end{array} \\
23.60\end{array}$} & \multirow{2}{*}{$\begin{array}{c}\begin{array}{c}\text { Mass } \\
(\mathrm{g})\end{array} \\
6.71\end{array}$} & \multirow{2}{*}{$\frac{\begin{array}{c}X \\
\left(10^{-6} \mathrm{~m}^{3} / \mathrm{kg}\right)\end{array}}{123.57}$} & \multirow{2}{*}{$\begin{array}{c}\begin{array}{c}X_{\mathrm{ARM}} \\
\left(10^{-6} \mathrm{~m}^{3} / \mathrm{kg}\right)\end{array} \\
13208.18\end{array}$} & \multirow{2}{*}{$\frac{X_{\mathrm{ARM}} / X}{106.89}$} & \multirow{2}{*}{$\begin{array}{c}\begin{array}{c}\text { SIRM } \\
(\mathrm{mA} / \mathrm{m} \cdot \mathrm{kg})\end{array} \\
41.87\end{array}$} & \multirow{2}{*}{$\begin{array}{c}\begin{array}{c}\text { HIRM } \\
(\mathrm{mA} / \mathrm{m} \cdot \mathrm{kg})\end{array} \\
52.92\end{array}$} & \multirow{2}{*}{$\frac{S}{0.983}$} \\
\hline $646 B$ & $3 \mathrm{H}$ & 7 & 20 & & & & & & & & \\
\hline $646 \mathrm{~B}$ & $3 \mathrm{H}$ & 7 & 40 & 23.80 & 6.46 & 126.02 & 13335.64 & 105.82 & 40.28 & 34.39 & 0.989 \\
\hline $646 B$ & $4 \mathrm{H}$ & 1 & 0 & 24.10 & 6.27 & 169.91 & 17608.78 & 103.64 & 57.47 & 58.52 & 0.987 \\
\hline $646 \mathrm{~B}$ & $4 \mathrm{H}$ & 1 & 10 & 24.20 & 6.53 & 181.23 & 17369.58 & 95.84 & 65.40 & 80.19 & 0.984 \\
\hline $646 \mathrm{~B}$ & $4 \mathrm{H}$ & 1 & 33 & 24.43 & 6.82 & 179.97 & 17407.60 & 96.73 & 67.44 & 110.46 & 0.978 \\
\hline $646 B$ & $4 \mathrm{H}$ & 1 & 53 & 24.63 & 6.71 & 127.13 & 12565.25 & 98.84 & 43.51 & 87.86 & 0.973 \\
\hline $646 \mathrm{~B}$ & $4 \mathrm{H}$ & 1 & 73 & 24.83 & 5.88 & 103.62 & 9656.92 & 93.19 & 29.70 & 46.70 & 0.982 \\
\hline $646 \mathrm{~B}$ & $4 \mathrm{H}$ & 1 & 93 & 25.03 & 5.84 & 142.84 & 12433.53 & 87.05 & 43.33 & 113.40 & 0.969 \\
\hline $646 \mathrm{~B}$ & $4 \mathrm{H}$ & 1 & 113 & 25.23 & 5.55 & 125.18 & 12692.29 & 101.40 & 37.99 & 146.39 & 0.957 \\
\hline $646 \mathrm{~B}$ & $4 \mathrm{H}$ & 1 & 133 & 25.43 & 6.22 & 122.19 & 13257.20 & 108.49 & 41.68 & 120.08 & 0.964 \\
\hline $646 \mathrm{~B}$ & $4 \mathrm{H}$ & 1 & 133 & 25.43 & 5.62 & 120.93 & 13322.17 & 110.16 & 36.87 & 99.73 & 0.970 \\
\hline $646 \mathrm{~B}$ & $4 \mathrm{H}$ & 2 & 0 & 25.60 & 6.00 & 106.99 & 11711.06 & 109.46 & 37.35 & 71.57 & 0.977 \\
\hline $646 \mathrm{~B}$ & $4 \mathrm{H}$ & 2 & 34 & 25.94 & 6.93 & 181.83 & 12191.01 & 67.05 & 56.68 & 62.29 & 0.985 \\
\hline $646 \mathrm{~B}$ & $4 \mathrm{H}$ & 2 & 53 & 26.13 & 6.31 & 229.95 & 13296.28 & 57.82 & 58.94 & 101.41 & 0.978 \\
\hline $646 \mathrm{~B}$ & $4 \mathrm{H}$ & 2 & 94 & 26.54 & 8.69 & 361.13 & 15117.88 & 41.86 & 140.04 & 203.49 & 0.975 \\
\hline $646 \mathrm{~B}$ & $4 \mathrm{H}$ & 2 & 110 & 26.70 & 8.03 & 226.07 & 15041.27 & 66.53 & 79.58 & 73.37 & 0.985 \\
\hline $646 \mathrm{~B}$ & $4 \mathrm{H}$ & 2 & 132 & 26.92 & 6.03 & 236.26 & 16510.00 & 69.88 & 58.04 & 43.85 & 0.991 \\
\hline $646 B$ & $4 \mathrm{H}$ & 2 & 132 & 26.92 & 6.96 & 243.13 & 16776.77 & 69.00 & 72.39 & 77.55 & 0.985 \\
\hline $646 \mathrm{~B}$ & $4 \mathrm{H}$ & 3 & 24 & 27.34 & 6.77 & 142.51 & 14496.60 & 101.72 & 45.08 & 19.01 & 0.994 \\
\hline $646 \mathrm{~B}$ & $4 \mathrm{H}$ & 3 & 47 & 27.57 & 5.91 & 134.98 & 15220.99 & 112.76 & 35.21 & 17.80 & 0.994 \\
\hline $646 \mathrm{~B}$ & $4 \mathrm{H}$ & 3 & 66 & 27.76 & 6.93 & 130.34 & 14037.54 & 107.70 & 39.01 & 73.14 & 0.974 \\
\hline $646 \mathrm{~B}$ & $4 \mathrm{H}$ & 3 & 105 & 28.15 & 6.91 & 146.54 & 13044.24 & 89.02 & 41.90 & 77.12 & 0.975 \\
\hline $646 \mathrm{~B}$ & $4 \mathrm{H}$ & 3 & 126 & 28.36 & 6.75 & 157.45 & 14352.88 & 91.16 & 44.73 & 74.11 & 0.978 \\
\hline $646 \mathrm{~B}$ & $4 \mathrm{H}$ & 3 & 126 & 28.36 & 6.91 & 140.54 & 13288.40 & 94.55 & 45.65 & 73.67 & 0.978 \\
\hline $646 \mathrm{~B}$ & $4 \mathrm{H}$ & 3 & 147 & 28.57 & 6.95 & 142.26 & 13970.03 & 98.20 & 47.64 & 72.02 & 0.979 \\
\hline $646 \mathrm{~B}$ & $4 \mathrm{H}$ & 4 & 10 & 28.70 & 6.95 & 133.04 & 11474.64 & 86.25 & 45.08 & 37.75 & 0.988 \\
\hline $646 \mathrm{~B}$ & $4 \mathrm{H}$ & 4 & 32 & 28.92 & 6.61 & 106.43 & 11311.47 & 106.28 & 35.83 & 26.38 & 0.990 \\
\hline $646 \mathrm{~B}$ & $4 \mathrm{H}$ & 4 & 50 & 29.10 & 6.46 & 149.35 & 12530.73 & 83.90 & 42.51 & 53.44 & 0.984 \\
\hline $646 \mathrm{~B}$ & $4 \mathrm{H}$ & 4 & 70 & 29.30 & 7.16 & 129.31 & 13106.91 & 101.36 & 47.63 & 64.13 & 0.981 \\
\hline $646 B$ & $4 \mathrm{H}$ & 4 & 90 & 29.50 & 6.65 & 118.26 & 12068.61 & 102.05 & 42.93 & 75.53 & 0.977 \\
\hline $646 \mathrm{~B}$ & $4 \mathrm{H}$ & 4 & 110 & 29.70 & 7.42 & 127.66 & 12343.39 & 96.69 & 48.51 & 68.12 & 0.979 \\
\hline $646 \mathrm{~B}$ & $4 \mathrm{H}$ & 4 & 130 & 29.90 & 7.04 & 116.35 & 10796.17 & 92.79 & 40.93 & 65.32 & 0.978 \\
\hline $646 B$ & $4 \mathrm{H}$ & 4 & 130 & 29.90 & 6.11 & 101.98 & 9904.88 & 97.12 & 30.71 & 58.17 & 0.977 \\
\hline $646 \mathrm{~B}$ & $4 \mathrm{H}$ & 5 & 4 & 30.14 & 6.25 & 131.86 & 13263.12 & 100.59 & 38.05 & 50.87 & 0.983 \\
\hline $646 \mathrm{~B}$ & $4 \mathrm{H}$ & 5 & 21 & 30.31 & 5.48 & 86.43 & 9633.48 & 111.46 & 23.44 & 43.28 & 0.980 \\
\hline $646 \mathrm{~B}$ & $5 \mathrm{H}$ & 1 & 0 & 33.80 & 6.89 & 132.56 & 11555.39 & 87.17 & 45.16 & 48.79 & 0.985 \\
\hline $646 \mathrm{~B}$ & $5 \mathrm{H}$ & 1 & 10 & 33.90 & 7.23 & 128.23 & 10501.69 & 81.89 & 46.31 & 66.47 & 0.979 \\
\hline $646 \mathrm{~B}$ & $5 \mathrm{H}$ & 1 & 30 & 34.10 & 7.01 & 153.76 & 13779.49 & 89.61 & 47.76 & 58.23 & 0.983 \\
\hline $646 B$ & $5 \mathrm{H}$ & 1 & 50 & 34.30 & 6.43 & 125.63 & 12709.35 & 101.17 & 36.71 & 104.74 & 0.963 \\
\hline $646 \mathrm{~B}$ & $5 \mathrm{H}$ & 1 & 90 & 34.70 & 7.42 & 114.28 & 11405.24 & 99.80 & 36.81 & 10.03 & 0.996 \\
\hline $646 \mathrm{~B}$ & $5 \mathrm{H}$ & 1 & 90 & 34.70 & 6.39 & 113.05 & 11322.85 & 100.16 & 31.40 & 86.42 & 0.965 \\
\hline $646 \mathrm{~B}$ & $5 \mathrm{H}$ & 1 & 117 & 34.97 & 7.16 & 153.53 & 15765.28 & 102.69 & 53.40 & 115.61 & 0.969 \\
\hline $646 \mathrm{~B}$ & $5 \mathrm{H}$ & 1 & 144 & 35.24 & 6.15 & 149.32 & 15660.21 & 104.87 & 41.88 & 91.15 & 0.973 \\
\hline $646 B$ & $5 \mathrm{H}$ & 2 & 14 & 35.44 & 7.46 & 87.57 & 14165.50 & 161.76 & 49.02 & 78.04 & 0.976 \\
\hline $646 \mathrm{~B}$ & $5 \mathrm{H}$ & 2 & 29 & 35.59 & 6.36 & 133.53 & 14108.87 & 105.66 & 39.64 & 61.97 & 0.980 \\
\hline $646 \mathrm{~B}$ & $5 \mathrm{H}$ & 2 & 74 & 36.04 & 7.77 & 136.62 & 13161.04 & 96.33 & 44.35 & 55.32 & 0.981 \\
\hline $646 B$ & $5 \mathrm{H}$ & 2 & 90 & 36.20 & 6.62 & 133.03 & 12231.28 & 91.94 & 37.50 & 63.50 & 0.978 \\
\hline $646 \mathrm{~B}$ & $5 \mathrm{H}$ & 2 & 112 & 36.42 & 3.00 & 117.25 & 13192.63 & 112.51 & 17.37 & 102.58 & 0.965 \\
\hline $646 \mathrm{~B}$ & $5 \mathrm{H}$ & 2 & 137 & 36.67 & 6.55 & 132.72 & 11659.06 & 87.84 & 41.30 & 85.93 & 0.973 \\
\hline $646 \mathrm{~B}$ & $5 \mathrm{H}$ & 3 & 0 & 36.80 & 6.83 & 138.32 & 13414.62 & 96.98 & 43.37 & 106.37 & 0.966 \\
\hline $646 \mathrm{~B}$ & $5 \mathrm{H}$ & 3 & 20 & 37.00 & 7.07 & 138.78 & 12562.46 & 90.52 & 47.41 & 113.30 & 0.966 \\
\hline $646 \mathrm{~B}$ & $5 \mathrm{H}$ & 3 & 42 & 37.22 & 7.23 & 121.46 & 11921.99 & 98.16 & 51.56 & 132.88 & 0.963 \\
\hline $646 \mathrm{~B}$ & $5 \mathrm{H}$ & 3 & 60 & 37.40 & 6.37 & 164.87 & 14290.45 & 86.67 & 57.72 & 162.31 & 0.964 \\
\hline $646 \mathrm{~B}$ & $5 \mathrm{H}$ & 3 & 80 & 37.60 & 6.39 & 122.68 & 10963.86 & 89.37 & 38.27 & 109.62 & 0.963 \\
\hline $646 \mathrm{~B}$ & $5 \mathrm{H}$ & 3 & 100 & 37.80 & 6.34 & 97.09 & 10163.59 & 104.68 & 34.00 & 108.19 & 0.960 \\
\hline $646 \mathrm{~B}$ & $5 \mathrm{H}$ & 3 & 120 & 38.00 & 6.16 & 103.60 & 10255.82 & 98.99 & 34.72 & 136.63 & 0.952 \\
\hline $646 B$ & $5 \mathrm{H}$ & 3 & 140 & 38.20 & 6.73 & 98.00 & 11073.01 & 112.99 & 41.20 & 153.02 & 0.950 \\
\hline
\end{tabular}




\begin{tabular}{|c|c|c|c|c|c|c|c|c|c|c|c|}
\hline \multicolumn{4}{|c|}{$\begin{array}{l}\text { Core, section } \\
\text { depth }(\mathrm{cm})\end{array}$} & \multirow{2}{*}{$\begin{array}{l}\begin{array}{l}\text { Depth } \\
\text { (mbsf) }\end{array} \\
38.20\end{array}$} & \multirow{2}{*}{$\begin{array}{c}\begin{array}{c}\text { Mass } \\
(\mathrm{g})\end{array} \\
6.33\end{array}$} & \multirow{2}{*}{$\frac{\begin{array}{c}X \\
\left(10^{-6} \mathrm{~m}^{3} / \mathrm{kg}\right)\end{array}}{104.99}$} & \multirow{2}{*}{$\begin{array}{c}\begin{array}{c}X_{\mathrm{ARM}} \\
\left(10^{-6} \mathrm{~m}^{3} / \mathrm{kg}\right)\end{array} \\
11671.31\end{array}$} & \multirow{2}{*}{$\frac{X_{\mathrm{ARM}} / X}{111.17}$} & \multirow{2}{*}{$\begin{array}{c}\begin{array}{c}\text { SIRM } \\
(\mathrm{mA} / \mathrm{m} \cdot \mathrm{kg})\end{array} \\
40.86\end{array}$} & \multirow{2}{*}{$\begin{array}{c}\begin{array}{c}\text { HIRM } \\
(\mathrm{mA} / \mathrm{m} \cdot \mathrm{kg})\end{array} \\
167.12\end{array}$} & \multirow{2}{*}{$\frac{S}{0.948}$} \\
\hline $646 B$ & $5 \mathrm{H}$ & 3 & 140 & & & & & & & & \\
\hline $646 B$ & $5 \mathrm{H}$ & 4 & 12 & 38.42 & 6.07 & 112.38 & 11575.59 & 103.00 & 41.42 & 166.25 & 0.951 \\
\hline $646 \mathrm{~B}$ & $6 \mathrm{H}$ & 1 & 7 & 43.47 & 7.24 & 118.17 & 10710.18 & 90.64 & 45.28 & 33.50 & 0.989 \\
\hline $646 \mathrm{~B}$ & $6 \mathrm{H}$ & 1 & 20 & 43.60 & 7.03 & 142.60 & 12084.28 & 84.74 & 50.49 & 41.58 & 0.988 \\
\hline $646 B$ & $6 \mathrm{H}$ & 1 & 40 & 43.80 & 7.44 & 113.64 & 10409.92 & 91.60 & 43.18 & 52.47 & 0.982 \\
\hline $646 \mathrm{~B}$ & $6 \mathrm{H}$ & 1 & 60 & 44.00 & 7.45 & 163.91 & 13531.94 & 82.56 & 74.21 & 33.38 & 0.993 \\
\hline $646 \mathrm{~B}$ & $6 \mathrm{H}$ & 1 & 80 & 44.20 & 7.48 & 109.00 & 10262.69 & 94.15 & 42.80 & 34.77 & 0.988 \\
\hline $646 \mathrm{~B}$ & $6 \mathrm{H}$ & 1 & 102 & 44.42 & 7.48 & 161.07 & 15026.00 & 93.29 & 59.27 & 119.00 & 0.970 \\
\hline $646 B$ & $6 \mathrm{H}$ & 1 & 120 & 44.60 & 6.94 & 137.58 & 14133.17 & 102.73 & 48.82 & 85.46 & 0.976 \\
\hline $646 \mathrm{~B}$ & $6 \mathrm{H}$ & 1 & 140 & 44.80 & 7.11 & 134.29 & 14901.87 & 110.97 & 49.95 & 77.32 & 0.978 \\
\hline $646 B$ & $6 \mathrm{H}$ & 1 & 140 & 44.80 & 6.20 & 139.20 & 15304.95 & 109.95 & 44.63 & 98.15 & 0.973 \\
\hline $646 B$ & $6 \mathrm{H}$ & 2 & 10 & 45.00 & 7.47 & 160.95 & 16344.61 & 101.55 & 64.08 & 95.37 & 0.978 \\
\hline $646 B$ & $6 \mathrm{H}$ & 2 & 30 & 45.20 & 7.58 & 142.53 & 15522.20 & 108.90 & 57.27 & 95.20 & 0.975 \\
\hline $646 \mathrm{~B}$ & $6 \mathrm{H}$ & 2 & 50 & 45.40 & 7.30 & 155.74 & 15196.53 & 97.57 & 57.78 & 100.53 & 0.975 \\
\hline $646 B$ & $6 \mathrm{H}$ & 2 & 74 & 45.64 & 7.65 & 175.39 & 17151.44 & 97.79 & 68.84 & 104.89 & 0.977 \\
\hline $646 \mathrm{~B}$ & $6 \mathrm{H}$ & 2 & 90 & 45.80 & 7.43 & 148.29 & 14944.68 & 100.78 & 59.78 & 112.40 & 0.972 \\
\hline $646 \mathrm{~B}$ & $6 \mathrm{H}$ & 2 & 110 & 46.00 & 6.64 & 146.63 & 14041.40 & 95.76 & 52.76 & 56.08 & 0.986 \\
\hline $646 \mathrm{~B}$ & $6 \mathrm{H}$ & 2 & 130 & 46.20 & 7.41 & 175.47 & 15740.82 & 89.71 & 65.72 & 52.78 & 0.988 \\
\hline $646 \mathrm{~B}$ & $6 \mathrm{H}$ & 2 & 130 & 46.20 & 6.62 & 161.49 & 15260.78 & 94.50 & 55.88 & 51. & 0.988 \\
\hline $646 \mathrm{~B}$ & $6 \mathrm{H}$ & 2 & 148 & 46.38 & 7.44 & 156.36 & 15124.85 & 96.73 & 57.50 & 46.68 & 0.988 \\
\hline $646 \mathrm{~B}$ & $6 \mathrm{H}$ & 3 & 0 & 46.40 & 6.49 & 140.73 & 13453.05 & 95.60 & 42.38 & 38.73 & 0.988 \\
\hline $646 \mathrm{~B}$ & $6 \mathrm{H}$ & 3 & 10 & 46.50 & 6.66 & 127.14 & 13424.86 & 105.59 & 44.43 & 62.88 & 0.981 \\
\hline $646 \mathrm{~B}$ & $6 \mathrm{H}$ & 3 & 60 & 47.00 & 7.49 & 101.14 & 9476.29 & 93.69 & 39.81 & 48.72 & 0.982 \\
\hline $646 \mathrm{~B}$ & $6 \mathrm{H}$ & 3 & 77 & 47.17 & 7.62 & 221.09 & 12849.19 & 58.12 & 76.69 & 87.82 & 0.983 \\
\hline $646 \mathrm{~B}$ & $6 \mathrm{H}$ & 3 & 100 & 47.40 & 8.10 & 187.67 & 15087.16 & 80.39 & 70.20 & 69.83 & 0.984 \\
\hline $646 \mathrm{~B}$ & $6 \mathrm{H}$ & 3 & 120 & 47.60 & 7.58 & 176.84 & 13558.39 & 76.67 & 71.77 & 102.32 & 0.978 \\
\hline $646 \mathrm{~B}$ & $6 \mathrm{H}$ & 3 & 120 & 47.60 & 7.97 & 177.33 & 13582.81 & 76.60 & 76.36 & 104.32 & 0.978 \\
\hline $646 B$ & $6 \mathrm{H}$ & 3 & 140 & 47.80 & 7.66 & 166.30 & 15633.32 & 94.01 & 69.49 & 84.26 & 0.981 \\
\hline $646 \mathrm{~B}$ & $6 \mathrm{H}$ & 4 & 10 & 48.00 & 6.73 & 133.28 & 12504.57 & 93.82 & 43.67 & 26.66 & 0.992 \\
\hline $646 \mathrm{~B}$ & $6 \mathrm{H}$ & 4 & 30 & 48.20 & 6.94 & 205.10 & 18193.27 & 88.71 & 75.95 & 69.61 & 0.987 \\
\hline $646 \mathrm{~B}$ & $6 \mathrm{H}$ & 4 & 50 & 48.40 & 7.49 & 259.31 & 21327.43 & 82.25 & 110.35 & 574.59 & 0.922 \\
\hline $646 \mathrm{~B}$ & $6 \mathrm{H}$ & 4 & 70 & 48.60 & 8.10 & 230.32 & 18606.61 & 80.79 & 104.89 & 529.12 & 0.918 \\
\hline $646 \mathrm{~B}$ & $6 \mathrm{H}$ & 4 & 90 & 48.80 & 6.93 & 194.33 & 17504.19 & 90.07 & 70.19 & 172.85 & 0.966 \\
\hline $646 \mathrm{~B}$ & $6 \mathrm{H}$ & 4 & 110 & 49.00 & 7.62 & 171.46 & 14192.85 & 82.78 & 69.17 & 201.99 & 0.955 \\
\hline $646 \mathrm{~B}$ & $6 \mathrm{H}$ & 5 & 0 & 49.40 & 6.36 & 139.26 & 13543.74 & 97.26 & 43.07 & 63.82 & 0.981 \\
\hline $646 B$ & $6 \mathrm{H}$ & 5 & 20 & 49.60 & 6.38 & 114.40 & 11868.91 & 103.75 & 38.96 & 69.47 & 0.977 \\
\hline $646 \mathrm{~B}$ & $6 \mathrm{H}$ & 5 & 60 & 50.00 & 7.59 & 153.93 & 12571.26 & 81.67 & 62.24 & 68.73 & 0.983 \\
\hline $646 \mathrm{~B}$ & $6 \mathrm{H}$ & 5 & 80 & 50.20 & 7.27 & 188.70 & 15372.25 & 81.46 & 71.78 & 129.27 & 0.974 \\
\hline $\begin{array}{l}646 B \\
646 B\end{array}$ & $\begin{array}{l}6 \mathrm{H} \\
6 \mathrm{H}\end{array}$ & $\begin{array}{l}5 \\
5\end{array}$ & $\begin{array}{l}100 \\
100\end{array}$ & $\begin{array}{l}50.40 \\
50.40\end{array}$ & $\begin{array}{l}7.81 \\
7.90\end{array}$ & $\begin{array}{l}184.50 \\
184.94\end{array}$ & $\begin{array}{l}15022.13 \\
14895.36\end{array}$ & $\begin{array}{l}81.42 \\
80.54\end{array}$ & $\begin{array}{l}71.52 \\
73.75\end{array}$ & $\begin{array}{l}130.66 \\
137.95\end{array}$ & $\begin{array}{l}0.971 \\
0.970\end{array}$ \\
\hline $646 \mathrm{~B}$ & $6 \mathrm{H}$ & 5 & 143 & 50.83 & 7.11 & 156.73 & 15478.24 & 98.76 & 53.71 & 29.85 & 0.992 \\
\hline $646 \mathrm{~B}$ & $6 \mathrm{H}$ & 6 & 24 & 51.14 & 7.90 & 148.53 & 15617.65 & 105.15 & 59.37 & 86.95 & 0.977 \\
\hline $646 \mathrm{~B}$ & $6 \mathrm{H}$ & 6 & 45 & 51.35 & 7.43 & 146.26 & 15379.56 & 105.15 & 55.37 & 92.28 & 0.975 \\
\hline $646 \mathrm{~B}$ & $6 \mathrm{H}$ & 6 & 61 & 51.51 & 7.55 & 168.56 & 18252.35 & 108.29 & 65.12 & 81.10 & 0.981 \\
\hline $646 \mathrm{~B}$ & $6 \mathrm{H}$ & 6 & 81 & 51.71 & 7.47 & 154.72 & 15802.41 & 102.13 & 55.80 & 99.75 & 0.973 \\
\hline $646 \mathrm{~B}$ & $6 \mathrm{H}$ & 6 & 100 & 51.90 & 7.72 & 141.74 & 14850.81 & 104.78 & 48.51 & 63.58 & 0.980 \\
\hline $646 \mathrm{~B}$ & $6 \mathrm{H}$ & 6 & 100 & 51.90 & 7.72 & 145.32 & 16393.82 & 112.81 & 53.73 & 46.20 & 0.987 \\
\hline $646 \mathrm{~B}$ & $6 \mathrm{H}$ & 6 & 121 & 52.11 & 7.61 & 172.35 & 18061.89 & 104.80 & 61.63 & 131.98 & 0.967 \\
\hline $646 \mathrm{~B}$ & $9 \mathrm{H}$ & 1 & 20 & 72.60 & 6.12 & 136.71 & 11714.82 & 85.69 & 36.08 & 73.12 & 0.975 \\
\hline $646 \mathrm{~B}$ & $9 \mathrm{H}$ & 1 & 30 & 72.70 & 6.67 & 146.72 & 14533.50 & 99.05 & 52.83 & 109.93 & 0.972 \\
\hline $646 \mathrm{~B}$ & $9 \mathrm{H}$ & 1 & 50 & 72.90 & 7.62 & 128.10 & 11048.85 & 86.25 & 52.00 & 93.75 & 0.973 \\
\hline $646 \mathrm{~B}$ & $9 \mathrm{H}$ & 1 & 89 & 73.29 & 8.37 & 173.06 & 11790.88 & 68.13 & 67.65 & 54.38 & 0.987 \\
\hline $646 \mathrm{~B}$ & $9 \mathrm{H}$ & 2 & 0 & 73.90 & 7.73 & 212.25 & 14371.66 & 67.71 & 75.60 & 103.25 & 0.979 \\
\hline $646 \mathrm{~B}$ & $9 \mathrm{H}$ & 2 & 10 & 74.00 & 7.76 & 190.38 & 14203.10 & 74.60 & 72.13 & 90.36 & 0.981 \\
\hline $646 \mathrm{~B}$ & $9 \mathrm{H}$ & 2 & 75 & 74.65 & 5.59 & 175.52 & 17503.66 & 99.72 & 48.47 & 85.10 & 0.980 \\
\hline $646 \mathrm{~B}$ & $9 \mathrm{H}$ & 2 & 91 & 74.81 & 6.72 & 200.78 & 21749.18 & 108.32 & 80.59 & 132.23 & 0.978 \\
\hline $646 \mathrm{~B}$ & $9 \mathrm{H}$ & 2 & 130 & 75.20 & 7.14 & 167.50 & 17643.19 & 105.33 & 68.41 & 112.37 & 0.977 \\
\hline
\end{tabular}


Appendix B (continued)

\begin{tabular}{|c|c|c|c|c|c|c|c|c|c|c|c|}
\hline \multicolumn{4}{|c|}{$\begin{array}{l}\text { Core, section } \\
\text { depth }(\mathrm{cm})\end{array}$} & \multirow{2}{*}{$\begin{array}{c}\begin{array}{c}\text { Depth } \\
\text { (mbsf) }\end{array} \\
75.40\end{array}$} & \multirow{2}{*}{$\begin{array}{c}\begin{array}{c}\text { Mass } \\
(\mathrm{g})\end{array} \\
7.94\end{array}$} & \multirow{2}{*}{$\frac{\begin{array}{c}X \\
\left(10^{-6} \mathrm{~m}^{3} / \mathrm{kg}\right)\end{array}}{117.56}$} & \multirow{2}{*}{$\begin{array}{c}\begin{array}{c}X_{\mathrm{ARM}} \\
\left(10^{-6} \mathrm{~m}^{3} / \mathrm{kg}\right)\end{array} \\
11142.61\end{array}$} & \multirow{2}{*}{$\frac{X_{\mathrm{ARM}} / X}{94.78}$} & \multirow{2}{*}{$\begin{array}{c}\begin{array}{c}\text { SIRM } \\
(\mathrm{mA} / \mathrm{m} \cdot \mathrm{kg})\end{array} \\
48.15\end{array}$} & \multirow{2}{*}{$\begin{array}{c}\begin{array}{c}\text { HIRM } \\
(\mathrm{mA} / \mathrm{m} \cdot \mathrm{kg})\end{array} \\
76.05\end{array}$} & \multirow{2}{*}{$\frac{S}{0.975}$} \\
\hline $646 \mathrm{~B}$ & $9 \mathrm{H}$ & 3 & 0 & & & & & & & & \\
\hline $646 B$ & $9 \mathrm{H}$ & 3 & 20 & 75.60 & 7.68 & 116.14 & 11180.90 & 96.27 & 44.74 & 39.75 & 0.986 \\
\hline $646 B$ & $9 \mathrm{H}$ & 3 & 41 & 75.81 & 7.34 & 134.53 & 12254.91 & 91.10 & 52.38 & 77.21 & 0.978 \\
\hline $646 \mathrm{~B}$ & $9 \mathrm{H}$ & 3 & 60 & 76.00 & 7.80 & 126.27 & 12407.23 & 98.26 & 55.20 & 64.17 & 0.982 \\
\hline $646 B$ & $9 \mathrm{H}$ & 31 & 120 & 76.60 & 7.60 & 138.52 & 14486.74 & 104.58 & 56.98 & 51.28 & 0.986 \\
\hline $646 \mathrm{~B}$ & $9 \mathrm{H}$ & 31 & 140 & 76.80 & 8.38 & 139.72 & 14219.97 & 101.77 & 54.87 & 29.22 & 0.991 \\
\hline $646 \mathrm{~B}$ & $9 \mathrm{H}$ & 4 & 0 & 76.90 & 7.56 & 158.86 & 17351.64 & 109.22 & 64.59 & 56.29 & 0.987 \\
\hline $646 \mathrm{~B}$ & $9 \mathrm{H}$ & 4 & 20 & 77.10 & 7.53 & 105.77 & 11916.32 & 112.66 & 46.63 & 49.54 & 0.984 \\
\hline $646 \mathrm{~B}$ & $9 \mathrm{H}$ & 4 & 40 & 77.30 & 8.23 & 101.05 & 10360.89 & 102.53 & 45.47 & 63.85 & 0.977 \\
\hline $646 \mathrm{~B}$ & $9 \mathrm{H}$ & 4 & 80 & 77.70 & 7.43 & 159.44 & 14228.44 & 89.24 & 64.78 & 119.82 & 0.973 \\
\hline $646 B$ & $9 \mathrm{H}$ & 41 & 100 & 77.90 & 8.21 & 128.84 & 11947.22 & 92.73 & 47.06 & -13.42 & 1.005 \\
\hline $646 \mathrm{~B}$ & $9 \mathrm{H}$ & 41 & 120 & 78.10 & 7.30 & 185.86 & 16781.68 & 90.29 & 67.27 & 55.14 & 0.988 \\
\hline $646 B$ & $9 \mathrm{H}$ & 41 & 120 & 78.10 & 6.51 & 185.45 & 17857.91 & 96.29 & 61.41 & 10.19 & 0.998 \\
\hline $646 \mathrm{~B}$ & $9 \mathrm{H}$ & 41 & 140 & 78.30 & 7.12 & 146.45 & 14470.88 & 98.81 & 56.55 & 41.34 & 0.990 \\
\hline $646 \mathrm{~B}$ & $9 \mathrm{H}$ & 5 & 10 & 78.50 & 7.79 & 124.34 & 12573.14 & 101.12 & 48.53 & -1.87 & 1.001 \\
\hline $646 \mathrm{~B}$ & $9 \mathrm{H}$ & 5 & 30 & 78.70 & 7.37 & 172.50 & 15137.42 & 87.75 & 71.84 & 27.47 & 0.994 \\
\hline $646 \mathrm{~B}$ & $9 \mathrm{H}$ & 5 & 68 & 79.08 & 7.34 & 163.28 & 13914.43 & 85.22 & 54.36 & 37.48 & 0.990 \\
\hline $646 \mathrm{~B}$ & $9 \mathrm{H}$ & 5 & 90 & 79.30 & 7.31 & 126.14 & 12129.22 & 96.15 & 55.79 & 89.40 & 0.977 \\
\hline $646 \mathrm{~B}$ & $9 \mathrm{H}$ & 51 & 112 & 79.52 & 7.57 & 166.45 & 14731.85 & 88.50 & 71.72 & 100.10 & 0.979 \\
\hline $646 \mathrm{~B}$ & $9 \mathrm{H}$ & 51 & 112 & 79.52 & 6.83 & 168.12 & 15247.36 & 90.69 & 65.28 & 161.74 & 0.966 \\
\hline $646 \mathrm{~B}$ & $9 \mathrm{H}$ & 6 & 0 & 79.90 & 8.05 & 200.69 & 15358.78 & 76.53 & 87.94 & 220.33 & 0.960 \\
\hline $646 \mathrm{~B}$ & $9 \mathrm{H}$ & 6 & 20 & 80.10 & 7.97 & 123.42 & 11853.18 & 96.04 & 45.80 & 14.24 & 0.995 \\
\hline $646 \mathrm{~B}$ & $9 \mathrm{H}$ & 6 & 40 & 80.30 & 7.79 & 187.56 & 17902.57 & 95.45 & 77.58 & 38.16 & 0.992 \\
\hline $646 \mathrm{~B}$ & $9 \mathrm{H}$ & 6 & 60 & 80.50 & 7.12 & 202.73 & 18926.62 & 93.36 & 77.94 & 22.25 & 0.996 \\
\hline $646 \mathrm{~B}$ & $9 \mathrm{H}$ & 61 & 120 & 81.10 & 6.48 & 205.70 & 20352.53 & 98.94 & 81.01 & 170.68 & 0.973 \\
\hline $646 \mathrm{~B}$ & $9 \mathrm{H}$ & 63 & 120 & 81.10 & 6.91 & 203.62 & 20127.41 & 98.85 & 83.37 & 128.35 & 0.979 \\
\hline $646 \mathrm{~B}$ & $9 \mathrm{H}$ & 61 & 140 & 81.30 & 7.25 & 136.54 & 13538.04 & 99.15 & 48.22 & 34.66 & 0.990 \\
\hline $646 B$ & $9 \mathrm{H}$ & 7 & 10 & 81.50 & 7.59 & 97.99 & 9350.11 & 95.42 & 33.50 & 35.66 & 0.984 \\
\hline $646 \mathrm{~B}$ & $9 \mathrm{H}$ & 7 & 30 & 81.70 & 7.48 & 124.28 & 11196.19 & 90.09 & 44.37 & 7.28 & 0.998 \\
\hline $646 \mathrm{~B}$ & $10 \mathrm{H}$ & 1 & 0 & 82.00 & 7.35 & 153.49 & 10948.80 & 71.33 & 51.83 & 114.33 & 0.968 \\
\hline $646 \mathrm{~B}$ & $10 \mathrm{H}$ & 1 & 20 & 82.20 & 7.54 & 71.98 & 6163.24 & 85.63 & 25.23 & 37.45 & 0.978 \\
\hline $646 B$ & $10 \mathrm{H}$ & 1 & 40 & 82.40 & 7.99 & 85.53 & 7433.96 & 86.91 & 33.57 & 39.75 & 0.981 \\
\hline $646 B$ & $10 \mathrm{H}$ & 1 & 60 & 82.60 & 7.55 & 130.62 & 11235.97 & 86.02 & 48.86 & 57.21 & 0.982 \\
\hline $646 \mathrm{~B}$ & $11 \mathrm{H}$ & 3 & 70 & 95.40 & 7.95 & 141.43 & 13801.87 & 97.59 & 58.49 & 91.47 & 0.975 \\
\hline $646 \mathrm{~B}$ & $11 \mathrm{H}$ & 3 & 90 & 95.60 & 6.63 & 138.70 & 14544.63 & 104.86 & 44.06 & 38.36 & 0.988 \\
\hline $646 B$ & $11 \mathrm{H}$ & 31 & 110 & 95.80 & 7.79 & 160.78 & 14844.28 & 92.32 & 77.29 & 77.08 & 0.984 \\
\hline $646 \mathrm{~B}$ & $11 \mathrm{H}$ & 31 & 130 & 96.00 & 7.83 & 184.03 & 16176.99 & 87.90 & 86.29 & 136.40 & 0.975 \\
\hline $646 \mathrm{~B}$ & $11 \mathrm{H}$ & 31 & 130 & 96.00 & 8.53 & 192.64 & 16200.73 & 84.10 & 91.28 & 177.54 & 0.967 \\
\hline $646 \mathrm{~B}$ & $12 \mathrm{H}$ & 1 & 40 & 101.80 & 7.82 & 136.07 & 15220.12 & 111.85 & 53.14 & 47.61 & 0.986 \\
\hline $646 B$ & $12 \mathrm{H}$ & 1 & 63 & 102.03 & 7.93 & 119.92 & 11054.33 & 92.18 & 44.75 & 66.00 & 0.977 \\
\hline $646 \mathrm{~B}$ & $12 \mathrm{H}$ & 1 & 81 & 102.21 & 7.88 & 135.83 & 12508.61 & 92.09 & 50.28 & 41.10 & 0.987 \\
\hline $646 \mathrm{~B}$ & $12 \mathrm{H}$ & 11 & 120 & 102.60 & 7.29 & 97.71 & 8474.99 & 86.74 & 30.23 & 11.26 & 0.995 \\
\hline $646 \mathrm{~B}$ & $12 \mathrm{H}$ & 11 & 120 & 102.60 & 6.56 & 107.44 & 8940.47 & 83.22 & 29.31 & 28.87 & 0.987 \\
\hline $646 \mathrm{~B}$ & $12 \mathrm{H}$ & 11 & 140 & 102.80 & 7.47 & 176.42 & 11805.01 & 66.92 & 60.64 & 84.85 & 0.979 \\
\hline $646 \mathrm{~B}$ & $12 \mathrm{H}$ & 2 & 6 & 102.96 & 8.85 & 198.45 & 13991.43 & 70.50 & 91.16 & 259.63 & 0.950 \\
\hline $646 \mathrm{~B}$ & $12 \mathrm{H}$ & 2 & 63 & 103.53 & 8.78 & 94.15 & 6279.40 & 66.70 & 32.88 & 16.47 & 0.991 \\
\hline $646 \mathrm{~B}$ & $12 \mathrm{H}$ & 21 & 105 & 103.95 & 7.45 & 129.00 & 11325.08 & 87.79 & 45.61 & 24.61 & 0.992 \\
\hline $646 \mathrm{~B}$ & $12 \mathrm{H}$ & 21 & 105 & 103.95 & 7.17 & 137.54 & 11489.98 & 83.54 & 46.94 & 83.03 & 0.975 \\
\hline $646 \mathrm{~B}$ & $12 \mathrm{H}$ & 21 & 145 & 104.35 & 7.59 & 166.18 & 13307.15 & 80.08 & 69.02 & 47.26 & 0.990 \\
\hline $646 \mathrm{~B}$ & $12 \mathrm{H}$ & 3 & 0 & 104.40 & 7.71 & 166.69 & 13440.91 & 80.63 & 72.10 & 61.07 & 0.987 \\
\hline $646 \mathrm{~B}$ & $12 \mathrm{H}$ & 3 & 20 & 104.60 & 7.55 & 175.55 & 15589.37 & 88.80 & 79.52 & 43.20 & 0.992 \\
\hline $646 \mathrm{~B}$ & $12 \mathrm{H}$ & 3 & 40 & 104.80 & 7.14 & 164.16 & 14762.36 & 89.93 & 67.59 & 183.53 & 0.961 \\
\hline $646 \mathrm{~B}$ & $12 \mathrm{H}$ & 3 & 60 & 105.00 & 8.04 & 130.32 & 12222.18 & 93.79 & 57.17 & 96.09 & 0.973 \\
\hline $646 \mathrm{~B}$ & $12 \mathrm{H}$ & 3 & 80 & 105.20 & 8.25 & 145.27 & 15001.07 & 103.26 & 67.63 & 112.21 & 0.973 \\
\hline $646 \mathrm{~B}$ & $12 \mathrm{H}$ & 3 & 100 & 105.40 & 7.58 & 213.47 & 18885.82 & 88.47 & 90.84 & 66.27 & 0.989 \\
\hline $646 B$ & $12 \mathrm{H}$ & 4 & 10 & 106.00 & 7.69 & 152.42 & 14804.35 & 97.13 & 65.45 & 123.59 & 0.971 \\
\hline $646 \mathrm{~B}$ & $12 \mathrm{H}$ & 4 & 30 & 106.20 & 7.50 & 129.65 & 13860.13 & 106.91 & 50.12 & 73.22 & 0.978 \\
\hline
\end{tabular}




\begin{tabular}{|c|c|c|c|c|c|c|c|c|c|c|c|}
\hline \multicolumn{4}{|c|}{$\begin{array}{l}\text { Core, section } \\
\text { depth }(\mathrm{cm})\end{array}$} & \multirow{2}{*}{$\begin{array}{r}\begin{array}{r}\text { Depth } \\
\text { (mbsf) }\end{array} \\
106.43\end{array}$} & \multirow{2}{*}{$\begin{array}{c}\begin{array}{c}\text { Mass } \\
(\mathrm{g})\end{array} \\
6.90\end{array}$} & \multirow{2}{*}{$\begin{array}{c}\begin{array}{c}X \\
\left(10^{-6} \mathrm{~m}^{3} / \mathrm{kg}\right)\end{array} \\
142.56\end{array}$} & \multirow{2}{*}{$\begin{array}{c}\begin{array}{c}X_{\mathrm{ARM}} \\
\left(10^{-6} \mathrm{~m}^{3} / \mathrm{kg}\right)\end{array} \\
13451.86\end{array}$} & \multirow{2}{*}{$\frac{X_{\mathrm{ARM}} / X}{94.36}$} & \multirow{2}{*}{$\begin{array}{c}\begin{array}{c}\text { SIRM } \\
(\mathrm{mA} / \mathrm{m} \cdot \mathrm{kg})\end{array} \\
50.29\end{array}$} & \multirow{2}{*}{$\begin{array}{c}\begin{array}{c}\text { HIRM } \\
(\mathrm{mA} / \mathrm{m} \cdot \mathrm{kg})\end{array} \\
168.59\end{array}$} & \multirow{2}{*}{$\frac{S}{0.954}$} \\
\hline $646 \mathrm{~B}$ & $12 \mathrm{H}$ & 4 & 53 & & & & & & & & \\
\hline $646 B$ & $12 \mathrm{H}$ & 4 & 68 & 106.58 & 7.49 & 187.52 & 18689.91 & 99.67 & 82.32 & 135.36 & 0.975 \\
\hline $646 B$ & $12 \mathrm{H}$ & 4 & 108 & 106.98 & 7.86 & 153.28 & 17244.30 & 112.50 & 69.22 & 29.73 & 0.993 \\
\hline $646 \mathrm{~B}$ & $12 \mathrm{H}$ & 4 & 108 & 106.98 & 6.63 & 154.05 & 17640.81 & 114.51 & 60.31 & 171.19 & 0.962 \\
\hline $646 \mathrm{~B}$ & $12 \mathrm{H}$ & 4 & 130 & 107.20 & 7.60 & 145.79 & 14704.44 & 100.86 & 60.93 & 67.84 & 0.983 \\
\hline $646 \mathrm{~B}$ & $12 \mathrm{H}$ & 4 & 148 & 107.38 & 7.87 & 134.89 & 13017.44 & 96.51 & 58.25 & 39.61 & 0.989 \\
\hline $646 B$ & $12 \mathrm{H}$ & 5 & 20 & 107.60 & 7.33 & 135.57 & 12850.06 & 94.79 & 54.59 & 46.43 & 0.988 \\
\hline $646 B$ & $12 \mathrm{H}$ & 5 & 40 & 107.80 & 7.54 & 151.95 & 14033.53 & 92.35 & 62.16 & 60.10 & 0.985 \\
\hline $646 B$ & $12 \mathrm{H}$ & 5 & 81 & 108.21 & 8.03 & 166.77 & 14033.74 & 84.15 & 70.26 & 20.40 & 0.995 \\
\hline $646 \mathrm{~B}$ & $12 \mathrm{H}$ & 5 & 120 & 108.60 & 7.60 & 105.46 & 11487.70 & 108.93 & 38.01 & 51.71 & 0.979 \\
\hline $646 B$ & $12 \mathrm{H}$ & 6 & 4 & 108.94 & 7.12 & 191.97 & 18510.57 & 96.42 & 75.39 & 127.43 & 0.976 \\
\hline $646 B$ & $12 \mathrm{H}$ & 6 & 34 & 109.24 & 7.36 & 204.32 & 18781.24 & 91.92 & 79.54 & 97.96 & 0.982 \\
\hline $646 B$ & $12 \mathrm{H}$ & 6 & 63 & 109.53 & 7.31 & 225.48 & 23838.07 & 105.72 & 89.80 & 186.30 & 0.970 \\
\hline $646 B$ & $13 \mathrm{H}$ & 1 & 10 & 111.20 & 6.71 & 84.25 & 8617.23 & 102.28 & 25.73 & -0.93 & 1.000 \\
\hline $646 B$ & $13 \mathrm{H}$ & 1 & 30 & 111.40 & 6.28 & 85.42 & 8351.67 & 97.77 & 24.81 & 37.59 & 0.981 \\
\hline $646 B$ & $13 \mathrm{H}$ & 1 & 52 & 111.62 & 7.00 & 114.14 & 9264.90 & 81.17 & 39.65 & 95.19 & 0.966 \\
\hline $646 B$ & $13 \mathrm{H}$ & 1 & 70 & 111.80 & 7.84 & 116.01 & 10099.93 & 87.06 & 41.12 & 46.49 & 0.982 \\
\hline $646 \mathrm{~B}$ & $13 \mathrm{H}$ & 1 & 120 & 112.30 & 7.80 & 116.93 & 11206.67 & 95.84 & 44.66 & 37.80 & 0.987 \\
\hline $646 B$ & $13 \mathrm{H}$ & 1 & 140 & 112.50 & 7.26 & 128.74 & 11863.03 & 92.15 & 44.24 & 45.67 & 0.985 \\
\hline $646 \mathrm{~B}$ & $13 \mathrm{H}$ & 1 & 140 & 112.50 & 7.11 & 126.33 & 11701.09 & 92.62 & 43.16 & 39.62 & 0.987 \\
\hline $646 \mathrm{~B}$ & $13 \mathrm{H}$ & 2 & 52 & 113.12 & 7.74 & 114.27 & 10884.20 & 95.25 & 37.77 & 9.85 & 0.996 \\
\hline $646 \mathrm{~B}$ & $13 \mathrm{H}$ & 2 & 80 & 113.40 & 8.53 & 183.66 & 14657.11 & 79.81 & 66.26 & 19.54 & 0.995 \\
\hline $646 \mathrm{~B}$ & $13 \mathrm{H}$ & 2 & 120 & 113.80 & 8.33 & 115.98 & 11394.91 & 98.25 & 39.93 & 27.65 & 0.988 \\
\hline $646 B$ & $13 \mathrm{H}$ & 2 & 140 & 114.00 & 7.39 & 96.73 & 9650.08 & 99.76 & 29.44 & 11.79 & 0.994 \\
\hline $646 \mathrm{~B}$ & $13 \mathrm{H}$ & 2 & 140 & 114.00 & 8.05 & 95.20 & 9593.31 & 100.77 & 32.75 & 35.20 & 0.983 \\
\hline $646 B$ & $13 \mathrm{H}$ & 3 & 37 & 114.47 & 6.63 & 188.54 & 15185.28 & 80.54 & 67.00 & 38.64 & 0.992 \\
\hline $\begin{array}{l}646 \mathrm{~B} \\
646 \mathrm{~B}\end{array}$ & $\begin{array}{l}13 \mathrm{H} \\
13 \mathrm{H}\end{array}$ & $\begin{array}{l}3 \\
3\end{array}$ & $\begin{array}{l}52 \\
70\end{array}$ & $\begin{array}{l}114.62 \\
114.80\end{array}$ & $\begin{array}{l}8.08 \\
7.57\end{array}$ & $\begin{array}{l}169.78 \\
154.84\end{array}$ & $\begin{array}{l}13845.37 \\
13427.94\end{array}$ & $\begin{array}{l}81.55 \\
86.72\end{array}$ & $\begin{array}{l}66.77 \\
60.58\end{array}$ & $\begin{array}{l}31.74 \\
52.78\end{array}$ & $\begin{array}{l}0.992 \\
0.987\end{array}$ \\
\hline $646 \mathrm{~B}$ & $13 \mathrm{H}$ & 3 & 105 & 115.15 & 7.63 & 107.85 & 9833.74 & 91.18 & 38.88 & 2.50 & 0.999 \\
\hline $646 B$ & $13 \mathrm{H}$ & 3 & 105 & 115.15 & 8.14 & 105.72 & 9608.24 & 90.88 & 40.11 & 0.98 & 1.000 \\
\hline $646 \mathrm{~B}$ & $13 \mathrm{H}$ & 4 & 0 & 115.60 & 7.45 & 107.92 & 9360.39 & 86.73 & 38.25 & 17.92 & 0.993 \\
\hline $646 B$ & $13 \mathrm{H}$ & 4 & 27 & 115.87 & 6.52 & 182.47 & 19413.40 & 106.39 & 66.18 & 53.99 & 0.989 \\
\hline $646 B$ & $13 \mathrm{H}$ & 4 & 40 & 116.00 & 6.91 & 173.99 & 17462.68 & 100.37 & 63.91 & 35.76 & 0.992 \\
\hline $646 \mathrm{~B}$ & $13 \mathrm{H}$ & 4 & 60 & 116.20 & 7.08 & 139.82 & 13797.98 & 98.68 & 50.14 & 40.57 & 0.989 \\
\hline $646 \mathrm{~B}$ & $13 \mathrm{H}$ & 4 & 80 & 116.40 & 7.74 & 141.86 & 14545.11 & 102.53 & 56.96 & 29.63 & 0.992 \\
\hline $646 \mathrm{~B}$ & $13 \mathrm{H}$ & 4 & 120 & 116.80 & 7.69 & 126.44 & 12558.73 & 99.32 & 57.28 & 82.32 & 0.978 \\
\hline $646 \mathrm{~B}$ & $13 \mathrm{H}$ & 4 & 140 & 117.00 & 7.33 & 135.05 & 13184.44 & 97.62 & 61.23 & 126.90 & 0.970 \\
\hline $646 B$ & $13 \mathrm{H}$ & 4 & 140 & 117.00 & 7.45 & 136.59 & 12747.97 & 93.33 & 60.66 & 118.96 & 0.971 \\
\hline $646 B$ & $13 \mathrm{H}$ & 5 & 10 & 117.20 & 7.10 & 135.36 & 11996.78 & 88.63 & 55.05 & 113.62 & 0.971 \\
\hline $646 B$ & $13 \mathrm{H}$ & 5 & 51 & 117.61 & 6.92 & 169.74 & 1669.29 & 9.83 & 64.01 & 105.61 & 0.977 \\
\hline $646 \mathrm{~B}$ & $13 \mathrm{H}$ & 5 & 71 & 117.81 & 8.09 & 147.21 & 12254.26 & 83.24 & 55.42 & 53.49 & 0.984 \\
\hline $646 B$ & $13 \mathrm{H}$ & 5 & 91 & 118.01 & 8.12 & 111.55 & 10665.55 & 95.61 & 42.01 & 57.62 & 0.978 \\
\hline $646 \mathrm{~B}$ & $13 \mathrm{H}$ & 6 & 10 & 118.70 & 7.67 & 177.88 & 14920.10 & 83.88 & 59.86 & 42.36 & 0.989 \\
\hline $646 \mathrm{~B}$ & $13 \mathrm{H}$ & 6 & 30 & 118.90 & 8.03 & 190.55 & 16938.21 & 88.89 & 63.86 & 49.26 & 0.988 \\
\hline $646 B$ & $13 \mathrm{H}$ & 6 & 53 & 119.13 & 7.45 & 184.82 & 19747.06 & 106.85 & 73.15 & 78.67 & 0.984 \\
\hline $646 \mathrm{~B}$ & $14 \mathrm{H}$ & 1 & 10 & 120.90 & 8.04 & 168.60 & 13555.50 & 80.40 & 71.64 & 57.12 & 0.987 \\
\hline $646 \mathrm{~B}$ & $14 \mathrm{H}$ & 1 & 32 & 121.12 & 7.51 & 172.97 & 14657.51 & $84 \cdot 74$ & 71.88 & 69.71 & 0.985 \\
\hline $646 \mathrm{~B}$ & $14 \mathrm{H}$ & 1 & 50 & 121.30 & 7.11 & 151.07 & 14136.08 & 93.57 & 54.87 & 53.57 & 0.986 \\
\hline $646 B$ & $14 \mathrm{H}$ & 1 & 70 & 121.50 & 8.78 & 123.48 & 11554.50 & 93.57 & 51.63 & 27.98 & 0.990 \\
\hline $646 \mathrm{~B}$ & $14 \mathrm{H}$ & 1 & 110 & 121.90 & 8.18 & 148.67 & 15738.23 & 105.86 & 62.41 & 52.70 & 0.986 \\
\hline $646 \mathrm{~B}$ & $14 \mathrm{H}$ & 1 & 110 & 121.90 & 8.25 & 146.34 & 15639.41 & 106.87 & 61.37 & 46.85 & 0.987 \\
\hline $646 B$ & $14 \mathrm{H}$ & 1 & 130 & 122.10 & 7.99 & 115.57 & 12476.18 & 107.96 & 47.23 & 44.78 & 0.985 \\
\hline $646 \mathrm{~B}$ & $14 \mathrm{H}$ & 1 & 148 & 122.28 & 7.83 & 124.51 & 13236.20 & 106.31 & 50.30 & 58.07 & 0.982 \\
\hline $646 B$ & $14 \mathrm{H}$ & 2 & 20 & 122.50 & 7.71 & 149.25 & 15455.03 & 103.55 & 62.15 & 69.13 & 0.983 \\
\hline $646 \mathrm{~B}$ & $14 \mathrm{H}$ & 2 & 41 & 122.71 & 7.37 & 163.30 & 16810.99 & 102.95 & 61.41 & 82.85 & 0.980 \\
\hline $646 \mathrm{~B}$ & $14 \mathrm{H}$ & 2 & 62 & 122.92 & 7.86 & 158.87 & 14921.62 & 93.92 & 65.09 & 84.78 & 0.980 \\
\hline $646 B$ & $14 \mathrm{H}$ & 2 & 75 & 123.05 & 8.03 & 150.03 & 13022.77 & 86.80 & 57.15 & 107.74 & 0.970 \\
\hline
\end{tabular}


Appendix B (continued)

\begin{tabular}{|c|c|c|c|c|c|c|c|c|c|c|c|}
\hline \multicolumn{4}{|c|}{$\begin{array}{c}\text { Core, section } \\
\text { depth }(\mathrm{cm})\end{array}$} & \multirow{2}{*}{$\begin{array}{r}\begin{array}{r}\text { Depth } \\
\text { (mbsf) }\end{array} \\
123.34\end{array}$} & \multirow{2}{*}{$\begin{array}{c}\begin{array}{c}\text { Mass } \\
(\mathrm{g})\end{array} \\
8.00\end{array}$} & \multirow{2}{*}{$\begin{array}{c}\begin{array}{c}X \\
\left(10^{-6} \mathrm{~m}^{3} / \mathrm{kg}\right)\end{array} \\
136.46\end{array}$} & \multirow{2}{*}{$\begin{array}{c}\begin{array}{c}X_{\mathrm{ARM}} \\
\left(10^{-6} \mathrm{~m}^{3} / \mathrm{kg}\right)\end{array} \\
12471.11\end{array}$} & \multirow{2}{*}{$\begin{array}{r}X_{\mathrm{ARM}} / X \\
91.39\end{array}$} & \multirow{2}{*}{$\begin{array}{c}\begin{array}{c}\text { SIRM } \\
(\mathrm{mA} / \mathrm{m} \cdot \mathrm{kg})\end{array} \\
47.12\end{array}$} & \multirow{2}{*}{$\begin{array}{c}\begin{array}{c}\text { HIRM } \\
(\mathrm{mA} / \mathrm{m} \cdot \mathrm{kg})\end{array} \\
45.83\end{array}$} & \multirow{2}{*}{$\frac{S}{0.984}$} \\
\hline $646 B$ & $14 \mathrm{H}$ & 2 & 104 & & & & & & & & \\
\hline $646 \mathrm{~B}$ & $14 \mathrm{H}$ & 2 & 120 & 123.50 & 7.84 & 166.17 & 16095.30 & 96.86 & 62.14 & 53.65 & 0.986 \\
\hline $646 B$ & $14 \mathrm{H}$ & 2 & 140 & 123.70 & 6.89 & 130.37 & 12866.76 & 98.70 & 51.73 & 87.87 & 0.977 \\
\hline $646 \mathrm{~B}$ & $14 \mathrm{H}$ & 2 & 140 & 123.70 & 6.80 & 130.43 & 13092.30 & 100.38 & 51.88 & 129.27 & 0.966 \\
\hline $646 B$ & $14 \mathrm{H}$ & 3 & 11 & 123.91 & 6.81 & 161.23 & 12507.10 & 77.57 & 48.44 & 68.98 & 0.981 \\
\hline $646 B$ & $14 \mathrm{H}$ & 3 & 40 & 124.20 & 7.92 & 201.77 & 14993.69 & 74.31 & 81.98 & 128.76 & 0.975 \\
\hline $646 B$ & $14 \mathrm{H}$ & 3 & 79 & 124.59 & 7.12 & 196.56 & 15046.09 & 76.55 & 69.93 & 74.12 & 0.985 \\
\hline $646 B$ & $14 \mathrm{H}$ & 3 & 133 & 125.13 & 7.57 & 141.39 & 12645.79 & 89.44 & 58.14 & 69.84 & 0.982 \\
\hline $646 B$ & $14 \mathrm{H}$ & 4 & 10 & 125.40 & 7.88 & 178.24 & 16689.73 & 93.64 & 70.68 & 64.00 & 0.986 \\
\hline $646 B$ & $14 \mathrm{H}$ & 4 & 30 & 125.60 & 7.34 & 114.67 & 11931.93 & 104.05 & 45.05 & 41.51 & 0.986 \\
\hline $646 B$ & $14 \mathrm{H}$ & 4 & 51 & 125.81 & 7.04 & 113.67 & 11626.85 & 102.28 & 42.32 & 64.15 & 0.979 \\
\hline $646 B$ & $14 \mathrm{H}$ & 4 & 70 & 126.00 & 7.61 & 110.61 & 9859.75 & 89.14 & 40.55 & 41.17 & 0.985 \\
\hline $646 B$ & $14 \mathrm{H}$ & 4 & 90 & 126.20 & 7.98 & 145.94 & 12275.19 & 84.11 & 54.96 & 6. & 0.984 \\
\hline $646 B$ & $14 \mathrm{H}$ & 4 & 110 & 126.40 & 7.34 & 165.34 & 15642.42 & 94.61 & 61.84 & 56.53 & 0.987 \\
\hline $646 \mathrm{~B}$ & $14 \mathrm{H}$ & 4 & 130 & 126.60 & 7.62 & 140.80 & 14052.88 & 99.81 & 59.52 & 50.39 & 0.987 \\
\hline $646 \mathrm{~B}$ & $14 \mathrm{H}$ & 4 & 130 & 126.60 & 7.62 & 137.17 & 13629.33 & 99.36 & 57.69 & 45.01 & 0.988 \\
\hline $646 \mathrm{~B}$ & $14 \mathrm{H}$ & 4 & 148 & 126.78 & 6.35 & 108.02 & 11017.09 & 101.99 & 40.09 & 50.22 & 0.984 \\
\hline $646 \mathrm{~B}$ & $14 \mathrm{H}$ & 5 & 31 & 127.11 & 7.52 & 132.14 & 12134.14 & 91.83 & 49.12 & 52.07 & 0.984 \\
\hline $646 \mathrm{~B}$ & $14 \mathrm{H}$ & 5 & 52 & 127.32 & 6.98 & 144.89 & 11991.01 & 82.76 & 47.75 & 32.59 & 0.990 \\
\hline $646 \mathrm{~B}$ & $14 \mathrm{H}$ & 5 & 93 & 127.73 & 8.07 & 207.82 & 15121.92 & 72.76 & 72.84 & 67.60 & 0.985 \\
\hline $646 \mathrm{~B}$ & $14 \mathrm{H}$ & 5 & 132 & 128.12 & 7.74 & 193.96 & 14810.16 & 76.36 & 62.41 & 23.40 & 0.994 \\
\hline $646 \mathrm{~B}$ & $14 \mathrm{H}$ & 5 & 132 & 128.12 & 7.74 & 191.85 & 14974.10 & 78.05 & 64.49 & 13.96 & 0.997 \\
\hline $646 \mathrm{~B}$ & $14 \mathrm{H}$ & 6 & 0 & 128.30 & 7.49 & 157.33 & 14074.21 & 89.46 & 50.02 & 30.13 & 0.991 \\
\hline $646 \mathrm{~B}$ & $14 \mathrm{H}$ & 6 & 20 & 128.50 & 7.65 & 160.11 & 15492.00 & 96.76 & 54.63 & 10.66 & 0.997 \\
\hline $646 \mathrm{~B}$ & $14 \mathrm{H}$ & 6 & 45 & 128.75 & 7.55 & 151.59 & 14057.71 & 92.74 & 53.20 & 34.80 & 0.990 \\
\hline $646 B$ & $14 \mathrm{H}$ & 6 & 74 & 129.04 & 8.09 & 105.91 & 10638.17 & 100.45 & 35.76 & 40.24 & 0.982 \\
\hline
\end{tabular}

